CAROLINA LA MAISON

Prevalência, continuidade e fatores de risco dos transtornos psiquiátricos na adolescência

Tese apresentada à Faculdade de Medicina da Universidade de São Paulo para obtenção do título de Doutor em Ciências

Programa de Saúde Coletiva

Orientadora: Prof ${ }^{\underline{a}}{ }^{-a}{ }^{\underline{a}}$ Alicia Matijasevich Manitto

SÃO PAULO

2019 
CAROLINA LA MAISON

\section{Prevalência, continuidade e fatores de risco dos transtornos psiquiátricos na adolescência}

Tese apresentada à Faculdade de Medicina da Universidade
de São Paulo para obtenção do título de Doutor em Ciências

Programa de Saúde Coletiva

Orientadora: Prof $^{\mathrm{a}} \mathrm{Dr}^{\mathrm{a}}$ Alicia Matijasevich Manitto

SÃO PAULO

2019 
Dados Internacionais de Catalogaçăo na Publicaçăo (CIP)

Preparada pela Biblioteca da

Faculdade de Medicina da Universidade de São Paulo

Creproduçăo autorizada pelo autor

Maison, Carolina La

Prevalência, continuidade e fatores de risco dos transtornos psiquiátricos na adolescência /

Carolina La Maison. -- São Paulo, 2019.

Tese (doutorado)--Faculdade de Medicina da Universidade de São Paulo.

Programa de Saúde Coletiva.

Orientadora: Alicia Matijasevich Manitto.

Descritores: 1.Prevalência 2. Fatores de risco 3.Adolescentes 4.Transtornos mentais 5.Estudos de coorte 6.Distúrbios do desenvolvimento neurológico

USP/FM/DBD-162/19

Responsável: Erinalva da Conceição Batista, CRB-8 6755 


\section{DEDICATÓRIA}

\section{A minha mamãe, Lorena.}

Minha doutora, na arte de ser mãe.

Eu não conseguiria sem a senhora.

A força do seu amor incondicional impulsionou cada sonho, cada página escrita nesta tese, cada artigo publicado.

Obrigada por acreditar nos meus sonhos, por ser minha melhor amiga e por sempre ter cuidado de mim, mesmo quando eu estava errada.

Obrigada por não ter desistido de mim. Obrigada por proporcionar financeira e psicologicamente a base para que eu conseguisse concretizar esse sonho. Obrigada por ser a melhor mãe do mundo e meu maior exemplo de pessoa. Obrigada, mamãe.

Todos os frutos colhidos nesses anos de mestrado e doutorado são seus.

E quando alcançarmos o objetivo de um "nome na porta de uma universidade", cada aluno orientado, cada pesquisa realizada, cada palestra ou trabalho apresentado, cada artigo publicado sempre será dedicado à senhora.

Esta conquista é mais sua do que minha. A doutora aqui é a senhora! Eu te amo mamãe. 


\section{DEDICATÓRIA}

\section{Em memória de José Valmir La Maison Júnior, O melhor irmão, filho, pai, amigo e homem que já conheci.}

Sei que está orgulhoso de mim e mesmo eu não o vendo, sei que está ao meu lado me protegendo e guiando.

Sinto saudades e estou cumprindo o que lhe prometi na última vez em que nos vimos: estou cuidando de mamãe e ela está bem orgulhosa. Ela sente sua falta todos os dias, eu também. Já se passaram muitos anos, mas eu ainda choro às vezes. Sei que você não deseja isso, mas é mais forte do que eu. Dói a ausência física. Mas a certeza do nosso encontro me conforta. Eu só tenho gratidão para você. Foi graças a sua entrega que eu pude ser quem eu sou. E foi graças a você que eu e mamãe nos unimos. Nos encontraremos um dia. Obrigada, meu irmão.

Te amo, Nenê. 


\section{AGRADECIMENTOS}

Agradeço especialmente

À Prof ${ }^{a}$ Drª Alicia Matijasevich Manitto, pela oportunidade de aprendizagem e de construção desta tese.

Por acreditar e confiar em mim, mesmo diante das adversidades. Pelo cuidado, seriedade e carinho com que conduziu os frutos desta tese e incentivou meu crescimento.

Pela atenção nos momentos difíceis enfrentados ao longo dos anos do doutorado, pelas palavras amigas e carinhosas. Tenho um carinho especial por você, professora, por tudo que me fez crescer e aprender ao longo desses anos. Sou grata e me sinto honrada de ter meu nome ao lado do seu. Admiro sua trajetória como pesquisadora e docente e, para mim, a senhora é um exemplo de dedicação e de quem quero ser.

Eu não conseguiria finalizar esta tese sem sua orientação. Muito obrigada por transformar um sonho em realidade e por mudar a minha vida desde nosso encontro na minha amada Argentina. Eu nunca vou esquecer a senhora e sempre serei grata por tudo que me proporcionou.

Que seja apenas o começo de muitos projetos juntas. 


\section{AGRADECIMENTOS}

A minha mamãe Lorena que tanto lutou para que este dia chegasse (sou doutora, mãe!).

A minha orientadora, Prof. Dra. Alicia Matijasevich, sem a senhora nada disso seria possível.

A minha vozinha, Maria Ortiz Falkembach, que sempre me incentivou e acreditou em mim. Te amo, vó!

Ao meu "paidrasto", Maurício Cossich, que cuidou de mamãe nesses anos dedicados ao estudo, proporcionando a paz que eu precisava para seguir em frente. Obrigada!

As minhas sobrinhas, Dayane La Maison e Luana La Maison. Vocês são a melhor parte do meu irmão, a melhor parte de mim. A tia ama demais vocês. Dayane me chame sempre de tia! Luana você sempre será a minha "pitita"!

A Laura La Maison Campestrini, o amor da minha vida. A razão dos meus sorrisos. Você deu sentido a tudo, pequena. Você é minha luz, minha paz. Sua chegada mudou a minha vida. A tia lhe ama tanto, mas tanto! Aos professores da banca de qualificação, Prof. Dr. Paulo Rossi, Prof. Dr. Wang Yuan Pange e Prof. Dr. Paulo Sergio Panse Silveira, pelas inestimáveis contribuições para a construção desta tese.

Agradeço também aos professores da banca de defesa, Prof. Dr. Paulo Rossi, Profa. Dra. Marly Augusto Cardoso e Profa. Dra. Luciana Tovo Rodrigues, por aceitarem dividir seus conhecimentos comigo e 
abrilhantarem ainda mais este momento tão importante e único na minha vida.

Aos professores do Departamento de Medicina Preventiva da Faculdade de Medicina da Universidade de São Paulo (FMUSP), pelos inúmeros aprendizados nesses anos de doutorado. Em especial à Prof. Dra. Marcia Thereza Couto Falcão, pela amizade, risadas, apoio e por ser um exemplo de mulher para mim. Admiro-a muito, professora.

Ao Prof. Dr. Mário César Scheffer que, ao compartilhar a sala com a minha orientadora, também participou de meu crescimento nesses anos de doutorado. Obrigada por sempre me incentivar. Sua presença deixa tudo mais leve.

À Prof. Dra. Ruth Terezinha Kehrig, minha eterna "melhor professora do mundo". Obrigada por sempre acreditar em mim. Eu estou aqui porque lá atrás a senhora estendeu a mão que eu precisava, contribuindo para que eu construísse meu caminho como pesquisadora. Te amo, professora.

À Prof. Dra Lucia Yasuko Izumi Nichiata que me orientou durante o mestrado e abriu as portas da Universidade de São Paulo para mim. Foi percorrendo esse caminho que cheguei até aqui. Gratidão eterna.

À Prof. Dra. Iná da Silva dos Santos que sempre me tratou com carinho e que, durante meu estágio em Pelotas, foi tão generosa e acolhedora. Obrigada por fazer parte dos produtos desta tese. Para mim é uma honra produzir com a senhora.

Ao Prof. Dr. Tiago Neuenfeld Munhoz, pelas contribuições nos produtos desta tese e por sua parceria nesse período do doutorado. 
Aos colaboradores dos manuscritos que compuseram esta tese. Obrigada pelas inestimáveis contribuições.

Às meninas da secretaria da pós-graduação da Faculdade de Medicina da Universidade de São Paulo, Lilian Santos de Godoy Prado, Marlene Goreti Sales e Juliana de Oliveira, por cada risada e incentivo nesses anos de doutorado. Tudo foi mais leve com a ajuda de vocês. Agradeço também a Maria Quitéria Paulino de Carvalho, sempre disposta a ajudar.

Aos meus colegas de departamento, em especial ao João Alexandre Mendes Teixeira e Waleska Regina Machado Araújo, pelo apoio e parceria desses anos. Muito obrigada!

A todos que trabalharam no acompanhamento aos 11 anos de idade da Coorte de Nascimentos de 2004. Em especial a Graciela Ferreira de Ferreira Mendes que me acolheu de uma forma tão generosa no meu estágio em Pelotas. Obrigada.

Ao Pós-Dr. Edvaldo Leal de Moraes (que orgulho um enfermeiro com seu currículo), gratidão por ter cruzado meu caminho e por ser tão generoso comigo. Espero que nós ainda produzamos muito juntos. Admiro-o e o respeito demais, você mora no meu coração.

À equipe da Organização de Procura de Órgãos (OPO), do Hospital das Clínicas, obrigada pela parceria e paciência - Nair Cordeiro dos Santos da Paixão gratidão por todos os ensinamentos. E obrigada Alexandre Chagas de Santana, pelas infinitas conversas sobre doutorado e sobre o amor pela docência - sempre serei grata pelos seus conselhos e generosidade e 
espero que nossa trajetória na docência ainda nos aproxime mais. Que venham o pós-doutorado e as alegrias e tormentas desse novo desafio.

Ao meu melhor amigo dessa "selva de pedra". Obrigada por mudar a minha vida e por cuidar de mim nessa "essepê". Te amo, José Antônio Furtado Segalla ou, como carinhosamente o chamo: te amo, Preto!

Aos meus irmãos de alma e coração, Paulo César e Hebert Ricci, a sua maneira, cada um contribuiu para que eu tivesse sabedoria para enfrentar os momentos de solidão e angústia. Obrigada pelo amor, apoio e incentivo. A vida ficou mais fácil depois que nos unimos. Vocês são parte disso tudo. Eu amo vocês!

Aos amigos que me ajudaram a enfrentar os dias cinza dessa "selva de pedra", alguns mais de perto, outros de longe, mas cada um com sua importância nesse processo: Andreia Zardim, Fabiana Martins, Helen Caldas e Evaristo, Leandro Muller, Luciano Borges, Maria Eduarda Dal Bello, Maria Ercília Paes de Barros, Mariane Mendonça, Murilo Pagani, Natalia Carvalho Leite, Sonia La Maison, Thayssa Ferraz.

A paz em pessoa, Larissa Sossai, e a dona da voz mais linda do sertão, Karola Nunes, pelas conversas infinitas, apoio e incentivo. Eu amo vocês! Ao querido amigo, Joel Luís Gonzaga da Silva, que nas minhas fugas sempre cuidou de mim. Obrigada a todos da "Barraca do Joel". A vida fica fácil quando encontramos pessoas do bem.

A la pareja Argentina que más amo. Hermanos para toda la vida: Graciela Inés Parrinello y Daniel Alberto Giachino. Gracias por los años de amistad 
y afecto. La Argentina está mucho más cerca después de nuestra amistad. Los quiero mucho.

A Elizabete dos Santos Vieira, pela parceria nesses últimos anos. Que mulher admirável é você! Que orgulho ter você por perto e saber que você faz parte da Universidade de São Paulo e de tudo o que esta universidade representa para mim. Muito obrigada!

Aos funcionários e amigos da Seção de Compras da FMUSP, Eliane Aparecida de Souza Cruz, Fábio Augusto Gomes Corrêa, Magno Masterson da Conceição, Ricardo Souza Santos, Wallace Lourenço Dantas, Wellington Antuany Ferreira Alves. Foi muito bom poder conviver com vocês nesses últimos anos. Obrigada por sempre estarem dispostos a ajudar e por me apoiarem e incentivarem para que eu terminasse esse sonhado doutorado.

A todos que trabalharam na Pesquisa de Avaliação do Impacto do Programa Criança Feliz, em especial a Daniela Ferreira Pauleti que foi tão competente e generosa para que esta pesquisa acontecesse - que Liz venha com saúde. A Luciane Pires Corrêa, pelas palavras sempre recheadas de incentivo e carinho. Obrigada!

A todas as mães e crianças da Coorte de Nascimentos de 2004 de PelotasRS, sem os quais este trabalho não seria possível.

A todos que de alguma forma contribuíram para a construção e conclusão desta tese. Muito obrigada.

Ao CNPq que financiou esses anos de estudo. A bolsa de doutorado foi imprescindível para que eu continuasse os estudos. 
Por fim, a Universidade de São Paulo. Que orgulho eu tenho de ser "cria" desta casa. Um dia ainda terei "meu nome em uma das suas portas. 
"Talvez não tenha conseguido fazer o melhor, Mas lutei para o que o melhor fosse feito. Não sou o que deveria ser, mas graças a Deus, não sou o que era antes" 
Esta tese está de acordo com as seguintes normas, em vigor no momento desta publicação:

Referências: adaptado de International Committee of Medical Journals Editors (Vancouver).

Universidade de São Paulo. Faculdade de Medicina. Divisão de Biblioteca e Documentação. Guia de apresentação de dissertações, teses e monografia. Elaborado por Anneliese Carneiro da Cunha, Maria Julia de A. L. Freddi, Maria F. Crestana, Marinalva de Souza Aragão, Suely Campos Cardoso, Valéria Vilhena. 3a ed. São Paulo: Divisão de Biblioteca e Documentação; 2011.

Abreviaturas dos títulos dos periódicos de acordo com List of Journals Indexed in Index Medicus. 


\section{Sumário}

1 INTRODUÇÃO

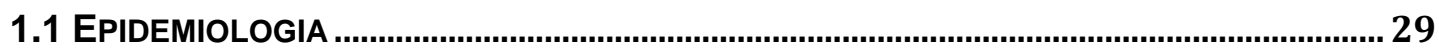

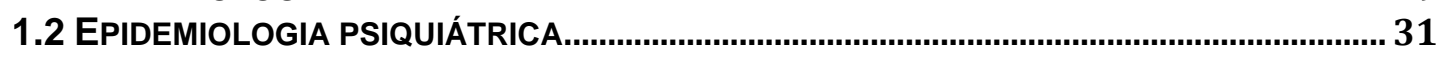

1.3. DEFINIÇÃO DE SAÚDE MENTAL E AS DIFERENTES CLASSIFICAÇÕES DIAGNÓSTICAS34

1.4. INSTRUMENTOS UTILIZADOS PARA DIAGNÓSTICO PSIQUIÁTRICO EM PESQUISAS..... 37

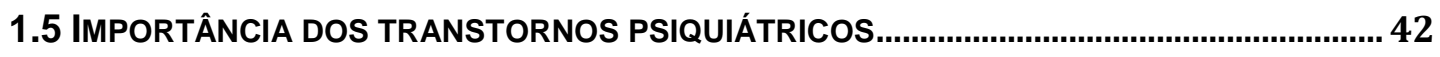

1.6. ESTUDOS DE PREVALÊNCIA EM SAÚDE MENTAL ..................................................... 45

1.7 PREVALÊNCIA DOS TRANSTORNOS PSIQUIÁTRICOS NA ADOLESCÊNCIA ......................46

1.8 CONTINUIDADE DOS TRANSTORNOS PSIQUIÁTRICOS NA ADOLESCÊNCIA...................52

1.9 FATORES DE RISCO DOS TRANSTORNOS PSIQUIÁTRICOS NA ADOLESCÊNCIA ........... 55

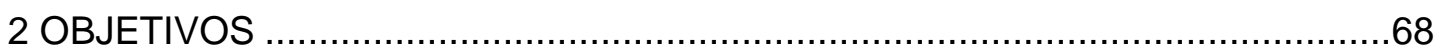

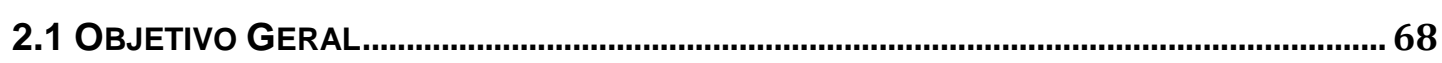

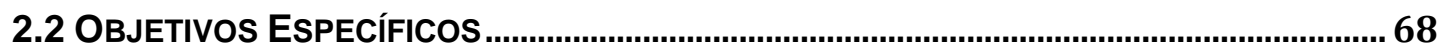

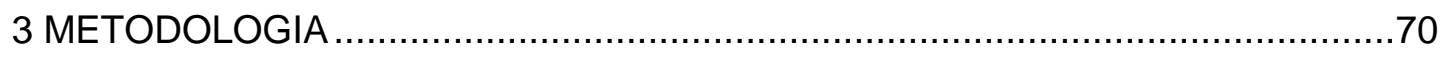

3.1. POPULAÇÃO ALVO

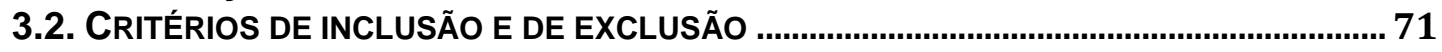

3.3. DEFINIÇÃO E OPERACIONALIZAÇÃO DO DESFECHO DO ESTUDO .................................... 72

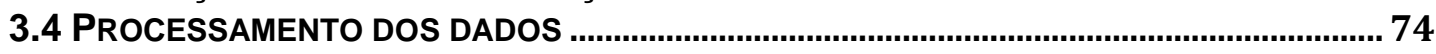

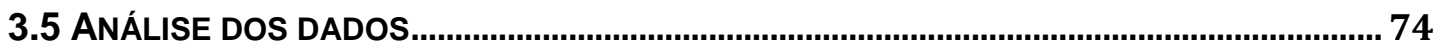

4 PARTICIPAÇÃO NO TRABALHO DE CAMPO …......................................76

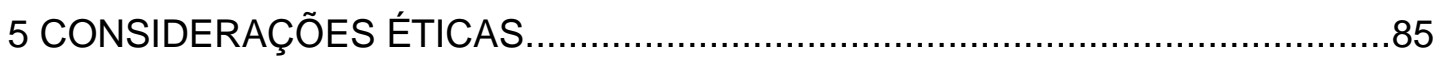

6. ARTIGO 1 - PREVALÊNCIA E FATORES DE RISCO DOS TRANSTORNOS PSIQUIÁTRICOS NO INÍCIO DA ADOLESCÊNCIA: COORTE DE NASCIMENTOS

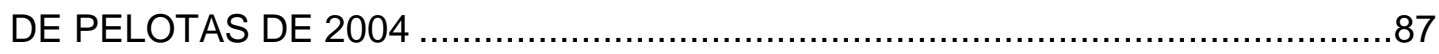

6.1 ABSTRACT

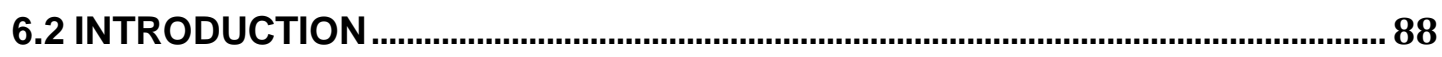

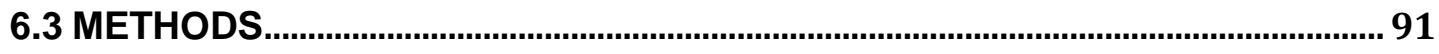

6.3.1 SETTING AND STUDY DESIGN ..................................................................................... 91

6.3.2 EVALUATION OF PSYCHIATRIC DISORDERS ……....................................................... 92

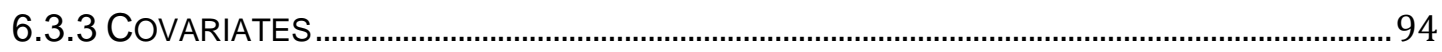

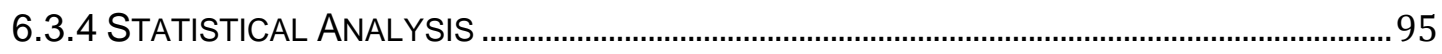

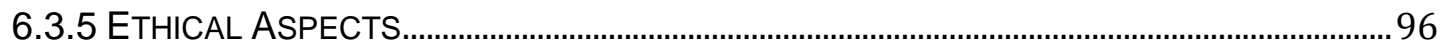

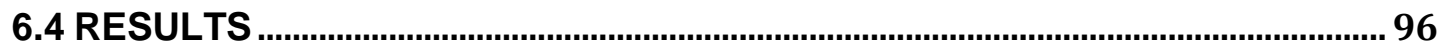

6.4.1 ATTRITION ANALYSIS AND CHARACTERISTICS OF THE SAMPLE....................................96

6.4.2 PREVALENCE OF PSYCHIATRIC DISORDERS ....................................................................97

6.4.3 RISK FACTORS FOR PSYCHIATRIC DISORDERS.......................................................... 98

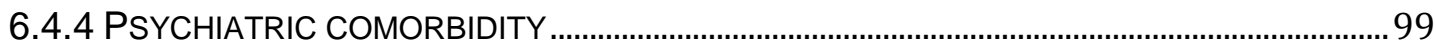




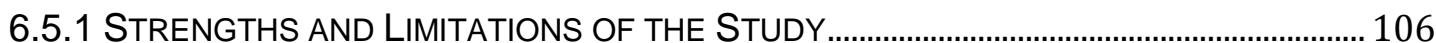

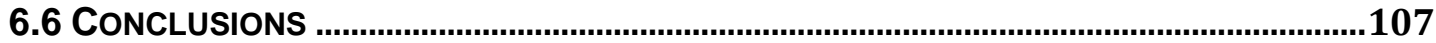

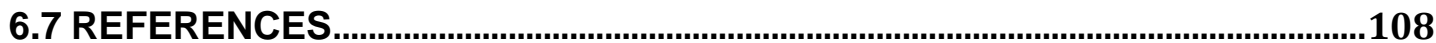

7. ARTIGO 2 - CONTINUIDADE DOS TRANSTORNOS MENTAIS ENTRE OS $6 \mathrm{E}$ 11 ANOS: COORTE DE PELOTAS DE 2004

7.1 RESULTS

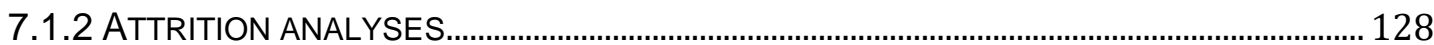

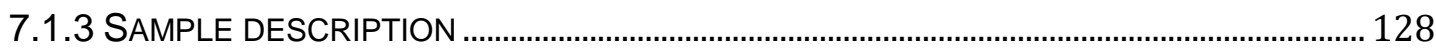

7.1.4 PREVALENCE, INCIDENCE AND PERSISTENCE OF MENTAL HEALTH PROBLEMS...... 128

7.1.5 HOMOTYPIC AND HETEROTYPIC CONTINUITY............................................................... 129

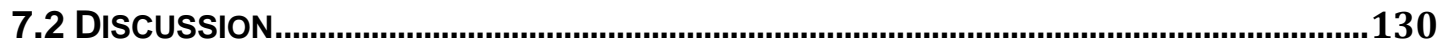

7.3 REFERENCES

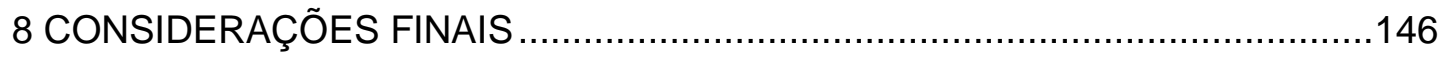

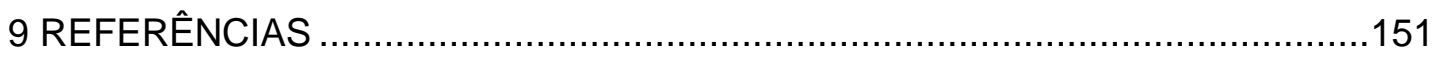




\section{LISTA DE QUADROS}

Quadro 1 - Descrição das entrevistas realizadas e das perdas em cada acompanhamento realizado na Coorte de Nascimentos de 2004 de PelotasRS. 


\section{LISTA DE FIGURAS}

Figura 1 - Modelo para entender os fatores de risco e de proteção dos transtornos psiquiátricos .58

Figura 2 - O ciclo da pobreza e do transtorno mental..................................65

Figura 3 - Modelo teórico dos fatores de risco..........................................66

Figura 4 - Assinatura do termo de divulgação de imagem............................78

Figura 5 - Equipe de entrevistadoras da coorte de 2004 após entrevista domiciliar 78

Figura 6 - Colocação do acelerômetro no punho do braço não dominante. Tal aparelho foi utilizado para mensuração objetiva de atividade física, captando os movimentos corporais para estimar os padrões de atividade física dos participantes

Figura 7 - Assinatura do TCLE 80

Figura 8 - Família da coorte de 2004 a caminho Centro de Pesquisa em Saúde "Dr. Amilcar Gigante" .81

Figura 9 - Preenchimento dos dados pessoais durante entrevista familiar da coorte de 2004

Figura 10 - Família chegando no Centro de Pesquisa em Saúde "Dr. Amilcar Gigante", para realizar os exames de pletismografia por deslocamento de ar (BodPod), densitometria (DXA), avaliação das dimensões corporais (Photonic Scanner), antropometria (altura, altura sentado e circunferência da cintura) e pressão arterial. .83 


\section{LISTA DE SIGLAS}

$\begin{array}{ll}\text { APA } & \text { American Psychological Association } \\ \text { BDD } & \text { Transtorno Dismórfico Corporal (Body Dysmorphic Disorder) } \\ \text { BED } & \text { Transtorno de compulsão alimentar (Binge Eating Disorder) } \\ \text { CBCL } & \begin{array}{l}\text { Inventário de Comportamentos da Infância e Adolescência } \\ \text { (Child Behavior Checklist) }\end{array} \\ \text { CGD } & \text { Carga Global de Doença (Global Burden of Disease- BD) } \\ \text { CID-10 } & \begin{array}{l}\text { Classificação Estatística Internacional de Doenças e } \\ \text { Problemas Relacionados à Saúde (International Statistical } \\ \text { Classification of Diseases and Related Health Problems - } \\ \end{array} \\ & \text { ICD) }\end{array}$

DAWBA Development and Well-Being Assessment

DALY Total de anos de vida perdidos ajustados por incapacidade (Disability-Adjusted Life Year)

DSM-IV e 5 Manual Diagnóstico e Estatístico de Transtornos Mentais (Diagnostic and Statistical Manual of Mental Disorders, fourth and Fifth Edition)

DMDD Transtorno Disruptivo da Desregulação do Humor (Disruptive Mood Dysregulation Disorder)

K-SADS-E Entrevista sobre Transtornos Afetivos e Esquizofrenia para Crianças em Idade Escolar, versão epidemiológica (KiddieSads-Present and Lifetime Version)

Erica Estudo de Riscos Cardiovasculares em Adolescentes

GHQ-12 General Health Questionnaire

OMS Organização Mundial da Saúde (World Health Organization WHO)

SDQ Questionário de Capacidades e Dificuldades (Strengths and Difficulties Questionnaire)

TDAH Transtorno do Déficit de Atenção com Hiperatividade (Attention Deficit Hyperactivity Disorder - ADHD)

YLD Total de anos vividos com incapacidade (Years Lived with Disability) 
Maison CL. Prevalência, continuidade e fatores de risco dos transtornos psiquiátricos na adolescência [tese]. São Paulo: Faculdade de Medicina, Universidade de São Paulo; 2019.

\section{RESUMO}

Os transtornos psiquiátricos frequentemente têm início na infância e adolescência, podendo persistir até a idade adulta. O objetivo da pesquisa foi estudar a prevalência, os fatores de risco e a continuidade dos transtornos psiquiátricos no início da adolescência (11 anos) na Coorte de Nascimentos de 2004 do Município de Pelotas-RS. Métodos. Estudo 1: O presente estudo teve como objetivos avaliar a prevalência de transtornos psiquiátricos no início da adolescência, examinar a distribuição dos transtornos psiquiátricos conforme características maternas e infantis e avaliar a ocorrência de comorbidades psiquiátricas. Todos os adolescentes de 11 anos, que participaram da Coorte de Nascimentos de 2004 de Pelotas-RS, foram convidados a participar deste estudo. $O$ instrumento utilizado para avaliar a presença de transtornos psiquiátricos foi 0 Development and Well-Being Assessment (DAWBA). Foram avaliados 3.562 indivíduos e a prevalência de transtornos psiquiátricos de acordo com os critérios do DSM-5 foi de 13,2\% (IC95\% 12,1-14,4); 15,6\% entre os meninos e $10,7 \%$ entre as meninas. Os distúrbios mais comuns foram transtornos de ansiedade $(4,3 \%)$, transtorno de déficit de atenção/hiperatividade $(4,0 \%)$ e transtorno de conduta/oposição (2,8\%). Baixa escolaridade materna, tabagismo durante a gestação, presença de sintomas de humor durante a gestação ou sintomas depressivos crônicos e graves maternos nos primeiros anos de vida do adolescente, sexo masculino, Apgar $<7$ no nascimento e parto prematuro foram associados a uma maior chance de distúrbio psiquiátrico aos 11 anos. Comorbidades psiquiátricas foram observadas em 107 indivíduos (22,7\%), dos quais, 73, 24 e 10 tinham dois, três e quatro diagnósticos psiquiátricos, respectivamente. Nossos resultados ressaltam a importância dos transtornos psiquiátricos como condição prevalente no início da adolescência, o que repercute diretamente no planejamento de políticas públicas e serviços específicos de atenção à saúde mental nessa faixa etária. Estudo 2: Os objetivos deste estudo foram investigar a incidência de transtornos psiquiátricos entre as idades de seis e 11 anos e avaliar a continuidade homotípica e heterotípica desses transtornos, entre os membros da Coorte de Nascimentos de 2004 de Pelotas-RS. Todos os nascidos vivos na cidade de Pelotas no ano de 2004 foram localizados e 4.231 recém-nascidos foram incluídos no estudo (recusas $<1 \%$ ), sendo acompanhados em diferentes idades ao longo do tempo. Aplicou-se o Strengths and Difficulties Questionnaire (SDQ) em 3.585 indivíduos com seis anos e em 3.563 indivíduos com 11 anos. Os resultados do SDQ para as quatro subescalas (sintomas emocionais, problemas de conduta, hiperatividade/falta de atenção e problemas de relacionamento com os pares) foram categorizados como normais ou anormais (indivíduos nas categorias limítrofe e anormal) conforme manual do instrumento. Para examinar as associações entre transtornos mentais ao longo do tempo, os 
transtornos aos seis anos foram inseridos na regressão logística como variáveis independentes e aqueles aos 11 anos foram inseridos como variáveis dependentes. Entre os seis e 11 anos, houve um aumento de $50 \%$ na prevalência de sintomas emocionais e um aumento de $45 \%$ dos transtornos de hiperatividade/falta de atenção. Entre as crianças que tinham "qualquer dificuldade no SDQ" aos seis anos, esse status persistiu em $81 \%$ dos indivíduos aos 11 anos. Durante a transição da infância para o início da adolescência, houve continuidade homotípica para sintomas emocionais, problemas de conduta, hiperatividade/falta de atenção e problemas de relacionamento com pares. Nossos resultados indicam que os transtornos mentais nessa faixa etária são moderadamente estáveis, com taxas de transtornos e padrões de continuidade semelhantes aos observados em outros estudos.

Descritores: Prevalência; Fatores de risco; Adolescentes; Transtornos mentais; Estudos de coorte; Distúrbios do desenvolvimento neurológico. 
Maison CL. Prevalence, continuity and risk factors of psychiatric disorders in adolescence [thesis]. São Paulo: "Faculdade de Medicina, Universidade de São Paulo"; 2019.

\section{ABSTRACT}

Psychiatric disorders often begin in childhood and adolescence and may persist into adulthood. The objective of the study was to study the prevalence, risk factors and continuity of psychiatric disorders in early adolescence (11 years) in the 2004 Birth Cohort of the Municipality of Pelotas, RS. Methods. Study 1: The present study aimed to evaluate the prevalence of psychiatric disorders in early adolescence, to examine the distribution of psychiatric disorders according to maternal and infant characteristics and to evaluate the occurrence of psychiatric comorbidities. All 11-year-old adolescents who participated in the 2004 Pelotas-RS Birth Cohort were invited to participate in this study. The instrument used to evaluate the presence of psychiatric disorders was the Development and Well-Being Assessment (DAWBA). A total of 3,562 individuals were evaluated and the prevalence of psychiatric disorders according to DSM-5 criteria was $13.2 \%(95 \% \mathrm{Cl} 12.1-14.4) ; 15.6 \%$ among boys and $10.7 \%$ among girls. The most common disorders were anxiety disorders $(4.3 \%)$, attention deficit/hyperactivity disorder (4.0\%) and conduct/opposition disorder (2.8\%). Low maternal schooling, smoking during pregnancy, presence of mood symptoms during pregnancy, or chronic and severe maternal depressive symptoms in the first years of the adolescent life, male sex, Apgar $<7$ at birth and premature delivery were associated with a greater chance of psychiatric disorder at age 11 years. Psychiatric comorbidities were observed in 107 subjects (22.7\%), of whom, 73, 24 and 10 had two, three and four psychiatric diagnoses, respectively. Our results highlight the importance of psychiatric disorders as a prevalent condition in early adolescence, which directly affects the planning of public policies and specific services for mental health care in this age group. Study 2: The objectives of this study were to investigate the incidence of psychiatric disorders between the ages of six and 11 years and to evaluate the homotypic and heterotypic continuity of these disorders among members of the 2004 Pelotas-RS Birth Cohort. All live births in the city of Pelotas in the year 2004 were located and 4,231 newborns were included in the study (refusals $<1 \%$ ), being followed at different ages over time. The Strengths and Difficulties Questionnaire (SDQ) was applied in 3,585 subjects aged 6 years and 3,563 subjects aged 11 years. The SDQ scores for the four subscales (emotional symptoms, behavioral problems, hyperactivity/inattention and peer relationship problems) were categorized as normal or abnormal (individuals in the borderline and abnormal categories) according to the instrument's manual. To examine the associations between mental disorders over time, disorders at six years were inserted into the logistic regression as independent variables and those at 11 years were entered as dependent variables. Between six and 11 years, there was a $50 \%$ increase in the prevalence of emotional symptoms and a $45 \%$ increase in hyperactivity/inattention 
disorders. Among children who had "any difficulty" according to the SDQ at age six, this status persisted in $81 \%$ of individuals at age 11 . During the transition from childhood to early adolescence, there was homotypic continuity for emotional symptoms, conduct problems, hyperactivity/inattention, and peer relationship problems. Our results indicate that mental disorders in this age group are moderately stable, with rates of disorders and patterns of continuity similar to those observed in other studies.

Descriptors: Prevalence; Risk factors; Adolescents; Mental disorders; Cohort studies; Neurodevelopmental disorders. 


\section{APRESENTAÇÃO}

Este projeto se insere na linha de pesquisa intitulada: "Transtornos mentais, consumo de substâncias e funções executivas no início da adolescência em uma coorte de nascimentos", coordenada pela $\operatorname{Prof}^{\mathrm{a}}$. $\mathrm{Dr}^{\mathrm{a}}$. Alicia Matijasevich. O estudo utilizou o banco de dados da Coorte de Nascimentos de 2004 de Pelotas-RS, resultante da aplicação do instrumento DAWBA junto às mães dos adolescentes integrantes da coorte, aos seis e aos 11 anos. O presente projeto faz parte do estudo de Coorte de Nascimentos de 2004 de Pelotas-RS, parcialmente financiado pelo Ministério da Saúde (Departamento de Ciência e Tecnologia - DECIT). O subprojeto responsável pela avaliação da saúde mental dos adolescentes aos 11 anos obteve financiamento específico da Fundação de Amparo à Pesquisa do Estado de São Paulo (Fapesp - Processo n. 2014/13864).

Os problemas de saúde mental em crianças e adolescentes têm alta prevalência, curso crônico e envolvem diversos determinantes, desde fatores genéticos, interação com os pais, até fatores sociodemográficos e culturais. Assim, a identificação da prevalência e dos fatores de risco dos transtornos psiquiátricos na infância e adolescência é importante para melhor compreender a etiologia destes transtornos e, dessa forma, planejar estratégias de intervenções curativas e preventivas específicas.

O estudo da prevalência dos transtornos psiquiátricos em crianças e adolescentes tem merecido destaque entre os pesquisadores da área da saúde mental, em diversos países ao redor do mundo. Entretanto, a 
investigação da continuidade e dos fatores de risco para os transtornos psiquiátricos em crianças e adolescentes tem sido menos frequente em países em desenvolvimento porque expressiva parte dos estudos é realizada por meio de delineamentos transversais. Os estudos de coorte permitem o cálculo de incidência das doenças ou eventos estudados e são os mais indicados para identificar os fatores de risco precoces.

Esta pesquisa, iniciada em 2015 com meu ingresso no doutorado junto ao Departamento de Medicina Preventiva da Universidade de São Paulo, utilizou os dados de um estudo de coorte de nascimentos, com o objetivo de estudar a prevalência, os fatores de risco e a continuidade dos transtornos psiquiátricos no início da adolescência (11 anos). Para isso, acompanhou-se a amostra $(n=3.566)$ de uma coorte de nascimentos brasileira (estudo da Coorte de Nascimentos de 2004 de Pelotas-RS).

No primeiro capítulo, expõe-se o contexto científico no qual se insere esta pesquisa. Inicialmente, apresenta-se uma visão geral sobre a epidemiologia e a epidemiologia psiquiátrica.

$\mathrm{Na}$ subseção "Definição de saúde mental e as diferentes classificações diagnósticas", apresenta-se as definições de saúde mental e os termos de classificações e diagnósticos na área da saúde mental.

$\mathrm{Na}$ subseção "Instrumentos utilizados para diagnóstico psiquiátrico em pesquisas", apresenta-se os diversos instrumentos de avaliação de psicopatologias em crianças e adolescentes. Faz-se ainda uma profunda explanação sobre o DAWBA, instrumento utilizado na pesquisa e que tem como base critérios diagnósticos padronizados e aceitos internacionalmente, 
com propriedades psicométricas adequadas, o que reforça a importância do presente estudo.

Na subseção "Importância dos transtornos psiquiátricos", apresentase inicialmente um panorama geral sobre a significativa proporção da carga global de doença (CGD), no que tange à saúde mental. O cenário é preocupante. Os transtornos neuropsiquiátricos (que incluem transtornos depressivos, transtorno bipolar, esquizofrenia, transtornos derivados do uso de álcool e drogas, transtornos de ansiedade, retardo mental e transtorno neurológicos específicos) representam 9,8\% da CGD em países de baixa e média renda. Nesse sentido, reconhecer o impacto dos problemas de saúde mental sobre a CGD e a relação entre os transtornos psiquiátricos e os processos que envolvem sua ocorrência é fundamental para o campo da saúde mental, na medida em que permite o desenvolvimento de políticas de intervenção, direcionadas à prevenção e/ou à promoção da saúde mental em crianças e adolescentes (World Health Organization [WHO], 2001).

Nas subseções "Estudos de prevalência em saúde mental" e "Prevalência dos transtornos psiquiátricos na adolescência", apresenta-se um breve histórico sobre o que é prevalência, além de se discutir as prevalências encontradas em estudos realizados em países de alta, média e baixa renda.

Nas subseções "Continuidade dos transtornos psiquiátricos na adolescência" e "Fatores de risco dos transtornos psiquiátricos na adolescência", discute-se a continuidade dos problemas de saúde mental na infância, na adolescência e na vida adulta, apresenta-se a definição de fator 
de risco e fator de proteção e traça-se uma relação dos fatores de risco mais frequentes. Para esta última seção também foi criado um modelo teórico, baseado na literatura e nos resultados encontrados nesta tese.

No Capítulo 2, descreve-se o objetivo da tese, de "estudar a prevalência, continuidade e fatores de risco dos transtornos mentais no início da adolescência (11 anos) na Coorte de Nascimentos de 2004 do Município de Pelotas-RS, Brasil", e os objetivos específicos.

No Capítulo 3, discorre-se sobre a metodologia utilizada nos produtos desta tese, a população alvo, os critérios de inclusão e de exclusão, a definição do desfecho do estudo e as demais variáveis estudadas, a metodologia do processamento dos dados e sua análise.

O Capítulo 4 traz uma explanação sobre a minha participação no trabalho de campo, durante a coleta de dados de indivíduos com 11 anos de idade da Coorte de Nascimentos de 2004 de Pelotas-RS, e sobre a contribuição que esta etapa trouxe para uma aproximação do delineamento e implementação de um estudo de coorte, além da valiosa contribuição que participar do trabalho de campo trouxe para a execução desta tese de doutorado.

Finalmente, apresenta-se os produtos construídos durante o período do doutorado, concretizados em dois manuscritos.

O manuscrito "Prevalence and risk factors of psychiatric disorders in earl adolescence: 2004 Pelotas (Brazil) birth cohort", que compõe o Capítulo 6 desta tese, avaliou a prevalência e os fatores de risco aos 11 anos de idade da Coorte de Nascimentos de 2004 de Pelotas-RS. Este manuscrito foi 
publicado na revista Social Psychiatry and Psychiatric Epidemiology, em 13 de abril de 2018, e corresponde ao primeiro e segundo objetivos específicos da presente tese.

O segundo manuscrito da tese, intitulado "Continuity of psychiatric disorders between 6 and 11 years of age: the 2004 Pelotas Birth Cohort", encontra-se no Capítulo 7. O trabalho foi submetido a BMC Psychiatryc e está em processo de avaliação pela revista. Neste manuscrito, discute-se o terceiro e o último objetivos específicos da tese: a continuidade dos transtornos psiquiátricos aos 11 anos dos adolescentes da Coorte de Nascimentos de 2004 de Pelotas-RS.

Nas considerações finais, discute-se as principais implicações dos achados encontrados nesta tese. Entende-se que os resultados aqui encontrados poderão contribuir para diminuir a lacuna de estudos de base populacional sobre transtornos psiquiátricos nos adolescentes de países de rendas média e baixa e para auxiliar no planejamento da oferta de serviços específicos para o atendimento dos problemas de saúde mental nessa faixa etária, minimizando o impacto dos transtornos a curto, médio e longo prazo. 
Embora ninguém possa voltar atrás e fazer um novo começo, qualquer um pode começar agora e fazer um novo fim".

Francisco do Espírito Santo 


\section{INTRODUÇÃO}

\subsection{Epidemiologia}

Segundo o Dicionário de Epidemiologia, de John M. Last et al. (2000), a epidemiologia é definida como o estudo da distribuição e determinantes de estados ou eventos relacionados à saúde em populações específicas e a aplicação deste estudo para controlar os problemas de saúde (Last, Spasoff et al. 2000). Um dos componentes da definição de Last é a mensuração da frequência de doença em populações ou grupos de pessoas, o que implica a quantificação da existência ou ocorrência de doenças ou outros eventos de interesse. Com base nessa informação é possível examinar a distribuição da existência ou ocorrência de doenças conforme as características das pessoas ou de grupos de pessoas.

A epidemiologia é, frequentemente, considerada a ciência básica da medicina preventiva e da saúde pública, mas seus princípios têm sido cada vez mais incorporados à pesquisa e à prática clínica. Historicamente, esteve associada ao estudo de doenças infectocontagiosas e ao estudo de doenças crônico-degenerativas, como os distúrbios cardiovasculares e o câncer. Mais recentemente, a epidemiologia também tem contribuído com a compreensão da etiologia e o controle de outros grupos de doenças, como os transtornos psiquiátricos e os agravos à saúde por causas externas, particularmente os relacionados à violência (Medeiros, 2006).

A partir dos anos 1960, a epidemiologia experimentou uma revolução. Com o advento da computação, ampliaram-se os bancos de dados e foram 
desenvolvidos métodos estatísticos mais eficazes para análise de múltiplas variáveis (Medronho, 2003).

Geralmente os grupos de pessoas utilizados em estudos epidemiológicos estão localizados em uma determinada área ou país, em um certo momento, com a definição de subgrupos de acordo com as características sociodemográficas, socioeconômicas, biológicas, hábitos pessoais ou exposição a um determinado fator ou intervenção. A comparação de dois ou mais grupos de pessoas que diferem quanto à distribuição de uma doença ou agravo à saúde permite a formulação de hipóteses sobre os possíveis fatores determinantes ou as causas das doenças ou agravos (Rothaman, 1998).

O processo de investigação epidemiológica inicia-se com a suspeita de que determinado fator influencia a ocorrência de doença, com base em observações clínicas, dados de rotina, exames laboratoriais, conhecimento gerado por outras áreas de investigação como, por exemplo, a pesquisa básica, ou especulações teóricas. Essa suspeita leva à formulação de uma hipótese, que é avaliada por um estudo epidemiológico especificamente elaborado para examinar tal questão.

Os dados são então coletados de forma sistemática e em seguida analisados para verificar se de fato existe uma associação entre o fator estudado e o evento de interesse. Caso esta associação seja observada, explicações alternativas como chance, erros sistemáticos ou efeito de variáveis adicionais, devem ser excluídas. Finalmente, avalia-se se a associação observada representa uma associação causal, com base em 
diversos critérios, como a temporalidade (a exposição deve preceder o efeito no tempo), a magnitude da associação, a consistência dos achados com resultados de estudos anteriores, a sua plausibilidade biológica (a associação é consistente com o conhecimento sobre a patogenia da doença), a existência de evidência experimental (a relação causal será mais forte se for comprovada em modelos experimentais) e a existência de gradiente biológico (o aumento da exposição implica em aumento do desfecho) (Hill AB, 1965; Medronho, 2003).

\subsection{Epidemiologia psiquiátrica}

A epidemiologia dos transtornos mentais, ou epidemiologia psiquiátrica, é o corpo de conhecimento que utiliza os princípios, conceitos, métodos e estratégias da investigação epidemiológica, para o estudo da saúde mental dos indivíduos, constituindo um campo que tem especial relevância desde as três últimas décadas (Medeiros 2006). Os transtornos psiquiátricos estão entre as mais sérias de todas as classes de doenças, devido a sua alta prevalência, sua cronicidade, à prematuridade de sua aparição na vida das pessoas e à gravidade das deficiências que elas produzem (OMS, 2000).

Após a segunda guerra mundial, houve um grande desenvolvimento da epidemiologia como ciência e, consequentemente, avançaram os estudos sobre prevalência de doenças mentais na população em geral. A partir dos anos 1960, o conhecimento na área da psiquiatria cresceu e, com este crescimento, aumentou também a necessidade de sistematizar a coleta de 
informações, desenvolvendo-se sistemas diagnósticos padronizados, como o DSM. Inicia-se, dessa forma, uma nova fase na história da epidemiologia psiquiátrica (Eaton, Merikangas, 2000). No começo dos anos 1980, um vasto estudo sobre prevalência de transtornos psiquiátricos na comunidade foi conduzido nos Estados Unidos. O Estudo da Área de Captação Epidemiológica do Instituto Nacional de Saúde Mental (ECA-NIMH) (Robbins, Regier, 1991; Weissman et al. 1996) foi a primeira grande pesquisa epidemiológica de base populacional com o objetivo de estimar a prevalência de transtornos mentais.

A aplicação das ferramentas da epidemiologia na psiquiatria levou ao desenvolvimento metodológico, incluindo a introdução de entrevistas de diagnóstico estruturadas e de métodos estatísticos para estimar a prevalência dos transtornos mentais e padrões de comorbidade, dentro e entre classes de transtornos, os correlatos sociodemográficos e ambientais e os fatores de risco para os transtornos mentais (Kessler, 2000; Merikangas et al. 2009). Na atualidade, mais de meio século após o início dos estudos de epidemiologia psiquiátrica, com a mudança do foco de atenção dos estudos de hospitais para os comunitários, apresenta-se um novo padrão de morbidade. Assim, cerca de $90 \%$ dos problemas mentais constituem-se de distúrbios não-psicóticos, principalmente a depressão e a ansiedade, incluindo a presença de sintomas como insônia, fadiga, irritabilidade, dificuldade de memória e de concentração (Maragno et al. 2008). Um dos desafios da epidemiologia psiquiátrica é entender como múltiplos fatores de 
risco interagem ao longo do tempo na produção de diferentes resultados na saúde mental (Eaton, Merikangas, 2000).

A epidemiologia psiquiátrica focada no estudo dos transtornos mentais que ocorrem na infância e adolescência é um campo relativamente novo, e a pesquisa nessa área vem crescendo também nas últimas décadas (Goodman, 2000). Existem três principais grupos diagnósticos na psiquiatria infantil: (1) desordens emocionais, também descritas como problemas internalizantes, a exemplo da depressão, ansiedade, desordens obsessivocompulsivas; (2) desordens de comportamento disruptivo, nomeadas também como problemas externalizantes, tais como conduta desafiadora excessiva, transtornos de conduta-agressividade em relação a pessoas e animais e comportamento transgressor, no qual as condutas estão mais dirigidas para o outro; e (3) transtornos do desenvolvimento como, por exemplo, problemas de aprendizagem e desordens autistas (Goodman, Scott, 2005).

A ocorrência de transtornos psiquiátricos em crianças e adolescentes é identificada com maior precisão por meio de estudos epidemiológicos de base populacional, que possibilitam investigar toda a população em risco e não apenas casos psiquiátricos de amostras clínicas. Os estudos epidemiológicos de base populacional permitem que inferências sejam feitas sobre a população total em risco, independentemente dos locais de atendimento em saúde mental (Fleming, Hsieh, 2002). Estudos dessa natureza apresentam resultados importantes para o direcionamento de políticas públicas voltadas à saúde mental infantil, auxiliando no 
planejamento e na implementação dos serviços de saúde oferecidos à população.

\subsection{Definição de saúde mental e as diferentes classificações diagnósticas}

De acordo com a Organização Mundial da Saúde (OMS) (WHO, 2001), o conceito de saúde mental abrange, dentre outros aspectos: "[...] o bem-estar subjetivo, a auto eficácia percebida, a autonomia, a competência, a dependência intergeracional e a autorrealização do potencial intelectual e emocional da pessoa". Ainda de acordo com esse documento, a saúde mental é determinante do bem-estar geral dos indivíduos e não pode ser compreendida unicamente como a ausência de transtornos mentais. As diferenças socioculturais e as diferenças de classes e gêneros devem ser consideradas quando se busca uma definição universal para a saúde mental.

Para realizar o diagnóstico de transtorno psiquiátrico existem critérios padronizados, como aqueles da Classificação Estatística Internacional de Doenças e Problemas Relacionados à Saúde (CID-10) ou os do DSM. Os dois sistemas de classificação (CID-10 e DSM) são aceitos internacionalmente e incluem, além dos critérios para diagnóstico de diferentes transtornos, o sofrimento e a incapacidade associados aos sintomas. Entretanto, são frequentes as críticas e insatisfação em relação a estes sistemas em razão de sua difícil aplicação no mundo real, onde os pacientes apresentam múltiplos conjuntos de sintomas que se enquadram 
em diversos diagnósticos, da falta de flexibilidade em relação à idade e ao sexo e da falta de sensibilidade quanto a questões culturais (Mash, Hunsley, 2007).

No ano de 2013, depois de mais de 10 anos de trabalho de especialistas de todo o mundo, foi publicado o DSM-5, que traz o que há de mais atual em termos de classificação e de diagnóstico na área da saúde mental, com novas definições e critérios diagnósticos, de acordo com os estudos mais recentes em psiquiatria (APA, 2014). O objetivo do DSM-5 é garantir que a nova classificação - com a inclusão, reformulação e exclusão de diagnósticos - forneça uma fonte segura e cientificamente embasada para aplicação em pesquisa e na prática clínica (Araújo, Neto, 2014). Embora o DSM-5 continue sendo uma classificação categórica de transtornos individuais, o manual reconhece que os transtornos psiquiátricos nem sempre se encaixam totalmente dentro dos limites de um único transtorno. Alguns domínios de sintomas, como depressão e ansiedade, envolvem múltiplas categorias diagnósticas e podem refletir vulnerabilidades subjacentes comuns a um grupo maior de transtornos (Araújo, Neto, 2014). O reconhecimento dessa realidade fez os transtornos inclusos no DSM-5 serem reordenados em uma estrutura organizacional revisada, com o intuito de estimular novas perspectivas clínicas.

Em seu aspecto estrutural, o DSM-5 rompeu com o modelo multiaxial introduzido na terceira edição do manual. As alterações do DSM-5, relativamente às anteriores, se deram tanto na estrutura, como no conteúdo (APA, 2013). Na estrutura, o sistema anterior de cinco eixos passou a ser 
organizado em capítulos, acabando com as negações que existiam no "Eixo 2". No que diz respeito às alterações de conteúdo, destacam-se a criação das novas categorias "obsessivo-compulsivo" e "perturbações relacionadas", bem como da categoria "perturbações relacionadas com trauma e estresse". Para além de inclusões, alterações e eliminações, o DSM-5 combinou vários diagnósticos. Assim, o termo "autismo" e "Síndrome de Asperger" desapareceram e foram englobados em um novo termo: "perturbação do espectro do autismo". Por sua vez, a "perturbação de aprendizagem específica" surgiu da combinação de três outras perturbações ("perturbação da leitura", "perturbação de matemática" e "perturbação da expressão escrita". Já a "perturbação delirante" surgiu da "perturbação psicótica compartilhada com perturbação delirante". De igual modo, foram ainda combinadas a "perturbação de pânico sem agorafobia" com a "perturbação de pânico com agorafobia", dando lugar simplesmente a "perturbação de pânico". Foram incluídos ainda novos diagnósticos como o transtorno dismórfico corporal, transtorno disruptivo da desregulação do humor (DMDD) e transtorno da compulsão alimentar periódica (APA, 2013).

Não há dúvida de que as mudanças no DSM-5 suscitaram polêmicas que dividiram a opinião de especialistas, no entanto. os pesquisadores do DSM-5 apontam que as modificações realizadas foram baseadas na melhor evidência científica disponível, que os critérios diagnósticos foram exaustivamente avaliados em estudos de campo, buscando verificar a utilidade, validade e confiabilidade de cada um deles e que os sintomas que 
suscitavam dúvidas foram trabalhados novamente de forma mais precisa (APA, 2013).

\subsection{Instrumentos utilizados para diagnóstico psiquiátrico em pesquisas}

As entrevistas estruturadas e os critérios de diagnóstico padronizados contribuem para aumentar a confiabilidade no diagnóstico de transtornos mentais no âmbito da pesquisa (Miranda et al. 2008). As escalas de rastreamento foram os primeiros instrumentos padronizados utilizados nos levantamentos epidemiológicos. Ao indivíduo é atribuído um escore total, geralmente resultado da soma das respostas positivas. Para cada escala, calcula-se o escore que melhor discrimine os indivíduos doentes daqueles não-doentes (Andreoli et al. 2000).

Diversos instrumentos de avaliação de psicopatologia em crianças e adolescentes foram desenvolvidos nos últimos 20 anos para aplicação na pesquisa. Na década de 1980, realizou-se um estudo pioneiro com o objetivo de desenvolver um instrumento de triagem, capaz de avaliar os transtornos mentais em crianças e adolescentes: o Questionário de Morbidade Psiquiátrica Infantil (QMPI), composto de 35 itens. Este instrumento, porém, não avaliava critérios de impacto dos transtornos (Almeida Filho, 1981).

Outro instrumento bastante usado como triagem de problemas de comportamento e emocionais em crianças e adolescentes é o Child Behavior Checklist (CBCL), desenvolvido por Achenbach (1991) e traduzido em mais de 79 idiomas. A versão brasileira do CBCL (4-18 anos) denomina-se 
Inventário de Comportamentos da Infância e Adolescência. O instrumento apresenta 118 itens com descrições de comportamentos que podem estar frequentemente presentes, algumas vezes presentes ou ausentes na vida da criança (Achenbach, 1991). No entanto, é importante lembrar que este instrumento identifica casos com sintomas significativos, mas não estabelece um diagnóstico psiquiátrico.

No ano de 1997, Robert Goodman propôs o SDQ ou Questionário de Capacidades e Dificuldades, desenhado para ser um questionário curto e simples, clinicamente útil e com boa aceitação pelos respondentes (Fleitlich, Goodman, 2000; Goodman, 1997). O SDQ é um questionário de rastreamento (screening) de problemas de saúde mental, proposto para avaliar o comportamento de crianças e adolescentes dos quatro aos 16 anos. Desde seu desenvolvimento, o SDQ tornou-se o instrumento de pesquisa mais amplamente utilizado para a detecção de problemas relacionados à saúde mental infanto-juvenil (Vostanis, 2006). O questionário está disponível gratuitamente em mais de 40 idiomas, incluindo o português (http://www.sdqinfo.com). Composto por 25 itens, sendo 10 itens sobre capacidades, 14 itens sobre dificuldades e um item neutro, o SDQ está dividido em cinco subescalas: sintomas emocionais, problemas de conduta, hiperatividade, problemas de relacionamento com colegas e comportamento pró-social. O instrumento é apresentado em três versões, indicadas para serem respondidas pelas próprias crianças (acima de 11 anos), por seus pais ou responsáveis e pelos professores. As alternativas para resposta apresentam como opções "falso" (zero ponto para este tipo de resposta), 
"mais ou menos verdadeiro" (um ponto) e "verdadeiro" (dois pontos), podendo ser assinalada apenas uma única opção por item. A pontuação total de dificuldades é gerada pela soma dos resultados das subescalas de condutas problemáticas, que são computados conforme instruções localizadas na página da internet do SDQ. O resultado pode variar de zero a 40, e a pontuação de cada uma das cinco subescalas pode variar de zero a 10, se todos os cinco itens forem completados. Todos os itens apresentam três possibilidades de pontuação: "falso", como zero; "mais ou menos verdadeiro", como 1; e "verdadeiro", como 2. A pontuação final pode identificar os casos como: normal, limítrofe ou desviante (Fleitlich-Bilyk, Goodman, 2004).

Outro instrumento de diagnóstico de transtornos mentais é o DAWBA, criado por Goodman et al. (2000), validado na população brasileira por Fleitlich-Bilyk, Goodman (2004). O sistema é composto por dois instrumentos: um questionário de rastreamento e uma entrevista de diagnóstico, constituída por uma seção de perguntas objetivas e outra de perguntas abertas sobre os sintomas e o impacto. No DAWBA, a entrevista está disposta em módulos e, em cada módulo, há regras claras e objetivas para continuar ou interromper a investigação, com base no número de sinais e sintomas presentes. Esse formato traz duas vantagens importantes em termos de facilidade e custo da aplicação: permite que o diagnóstico seja feito diretamente, a partir das informações sobre os sintomas e seu impacto fornecidas pelo entrevistado (pais ou responsáveis, professor ou o próprio adolescente); e o entrevistador não necessita de background clínico, mas 
apenas de habilidades básicas para conduzir a entrevista e um treinamento específico no instrumento. A análise das respostas fechadas e abertas deve ser feita por um clínico que, segundo sua experiência, obterá o diagnóstico conforme as classificações diagnósticas (Fleitlich-Bilyk, Goodman, 2004). O DAWBA foi concebido para gerar diagnósticos psiquiátricos de acordo com os critérios da CID-10 e do DSM para idades de cinco a 17 anos (APA, 2014). O DAWBA tem três versões: uma para pais, uma para crianças com idade igual ou superior a 11 anos e uma para professores. A versão destinada aos pais envolve 14 seções de perguntas com alternativas de resposta e uma seção adicional de perguntas abertas. Cada uma das 14 seções diz respeito a uma classe de problema em saúde mental: desenvolvimento; ansiedade de separação; fobia específica; fobia social; pânico e agorafobia; estresse pós-traumático; transtorno obsessivocompulsivo; ansiedade generalizada; depressão; transtorno de atenção e de hiperatividade: transtorno desafiador de oposição; transtorno de conduta; transtornos alimentares; transtornos de tiques e outras preocupações. Para cada um destes transtornos, a entrevista investiga os sintomas e outros critérios necessários, utilizando a CID-10 e o DSM-5. Todas as 14 seções do DAWBA apresentam uma estrutura semelhante: uma breve introdução para o entrevistado, sobre o que será tratado naquela seção, e uma ou duas perguntas de screening, para verificar se é adequado prosseguir na seção. O entrevistado é então questionado, em detalhes, sobre a presença, severidade, duração e início dos sintomas relacionados ao problema de saúde mental investigado na seção. Quando o número de sintomas e a 
duração atingem o critério estipulado no instrumento, o protocolo orienta o entrevistador a marcar na página final a presença de determinada área de dificuldade.

Existem também os instrumentos que avaliam transtornos mentais específicos. Um exemplo instrumento é a Entrevista sobre Transtornos Afetivos e Esquizofrenia para Crianças em Idade Escolar, versão epidemiológica (Kiddie-Sads-Present and Lifetime Version - K-SADS-E). O instrumento foi construído para verificar e registrar episódios psicopatológicos em crianças e adolescentes com idade de seis a 17 anos, segundo critérios DSM-III (APA, 1980) e DSM-III-R. Este instrumento de entrevista foi desenvolvido por Orvaschel (1994) e traduzido no Brasil por Mercadante et al. (1995). No ano de 2003, a versão do K-SADS foi traduzida e suas propriedades psicométricas avaliadas (Brasil, 2003).

Existe uma diversidade de escalas e questionários utilizados nos diferentes países e uma grande variação na maneira de se definir a presença ou ausência de transtornos mentais. Taxas mais altas de transtornos psiquiátricos são observadas em estudos que aplicam instrumentos de screening. Uma revisão sistemática e meta-análise da prevalência do transtorno depressivo maior, abrangendo 116 estudos, constatou que pesquisas que utilizaram escalas de sintomas relataram uma prevalência mais elevada de transtorno depressivo do que aquelas em que foram utilizados critérios como o DSM-IV ou CID-10 (Ferrari et al. 2013).

Os instrumentos de avaliação dos transtornos mentais podem gerar classificações dimensionais ou categóricas. As classificações dimensionais 
produzem um escore com medidas contínuas da psicopatologia. As avaliações categóricas geram uma classificação da psicopatologia de forma não contínua, de acordo com critérios diagnósticos pré-estabelecidos, geralmente da DSM ou CID-10. Por exemplo, os diagnósticos do DAWBA são fornecidos de forma dicotômica "sim" ou "não", respeitando-se estritamente os critérios diagnósticos definidos pela CID-10 e DSM (Buka et al. 2002). Além do uso em categorias, é possível usar o DAWBA de forma dimensional, por meio do escore contínuo. A forma dimensional produz um escore com medidas contínuas dos problemas de comportamento. Entretanto, na forma categórica, a medida gera uma classificação de acordo com o ponto de corte pré-estabelecido (Goodman, Scott, 2005).

\subsection{Importância dos transtornos psiquiátricos}

Os problemas de saúde mental são responsáveis por uma proporção significativa da CGD em todo o mundo (Kielling et al. 2011; Whitheford et al. 2010). Os transtornos neuropsiquiátricos - transtorno depressivo unipolar, transtorno bipolar, esquizofrenia, epilepsia, transtornos derivados do uso de álcool e drogas, demência, transtornos de ansiedade, retardo mental e transtornos neurológicos específicos - representam 9,8\% da CGD em países de baixa e média renda. A adição de lesões autoinfligidas aumenta essa proporção para 11,1\% (Patel, 2007). Quase três quatros da carga global de transtornos neuropsiquiátricos ocorrem em países de rendas média e baixa (OMS, 2008). Nos países de renda baixa, a CGD causada pela depressão é quase a mesma que a causada pela malária $(3,2 \%$ vs. 
4,9\% da CGD). Nos países de renda média, a depressão é a causa que mais contribui para a CGD. O impacto dos transtornos mentais amplia-se muito além dos cálculos de carga de doenças. As pessoas com doença mental têm maior possibilidade de desenvolver problemas de saúde como diabetes, doenças cardíacas, acidente vascular cerebral, problemas respiratórios, doenças sexualmente transmissíveis e têm maior risco de morte prematura em comparação com a população em geral (OMS, 2010).

Os transtornos psiquiátricos nos adolescentes causam sofrimento pessoal e familiar e constituem fatores de risco reconhecidos para o abuso de substâncias e a criminalidade, além de predizerem desfechos negativos na idade adulta (De Girolamo et al. 2012). Uma proporção significativa dos diagnósticos psiquiátricos identificados em adultos tem suas raízes na infância e na adolescência (Kessler et al. 2007; Gore et al. 2011). Estudos evidenciaram que, mundialmente, cerca de $20 \%$ das crianças e adolescentes sofrem de alguma doença mental incapacitante, sendo o suicídio a terceira principal causa de morte entre os adolescentes. Além disso, aproximadamente metade de todas as doenças mentais dos adultos tem início na adolescência (OMS, 2008; Belfer et al. 2008). Os problemas de saúde mental de início precoce também estão associados a fatores de risco mais graves na infância e adolescência e a piores prognósticos na vida adulta (Moffitt, Caspi 2001; Moffitt et al. 2002). Em 2010, os transtornos mentais foram responsáveis por $7,4 \%$ do total de anos de vida perdidos ajustados por incapacidade (Disability-Adjusted Life Year - DALY) e 22,9\% do total de anos vividos com incapacidade (Years Lived with Disability - 
YLD), tornando-se a quinta principal causa de DALY e a primeira causa de YLD no mundo (Whiteford et. al., 2010).

Entre jovens com idade de 10 a 24 anos, os transtornos neuropsiquiátricos são responsáveis por $45 \%$ dos anos de DALY. Embora parte dos problemas de saúde mental ocorra após a infância e a adolescência, grande número de indivíduos vai continuar a apresentar transtornos semelhantes (continuidade homotípica) ou novos (continuidade heterotípica) depois de chegar à idade adulta. Nesse sentido, pode-se argumentar que grande parte da carga de doença imposta por transtornos mentais, neurológicos e por uso de substâncias em todo o mundo pode ser atribuída à incidência de transtornos mentais durante a infância e a adolescência e a sua persistência até idades mais avançadas (Kieling, Belfer, 2012). Mesmo não estando entre as principais causas diretas de mortalidade, as doenças mentais neurológicas e por uso de substâncias já representavam 12,3\% da CGD no ano de 2000, percentual que deve aumentar para 16,4\% em 2030 (Mathers, Loncar, 2006).

O reconhecimento do impacto dos problemas de saúde mental sobre a CGD e a relação entre os transtornos mentais e os processos que envolvem sua ocorrência são fundamentais para o campo da saúde mental, na medida em que permitem o desenvolvimento de políticas de intervenção direcionadas à prevenção e/ou à promoção da saúde mental de crianças e adolescentes (OMS, 2001; Sá et. al., 2010). 


\subsection{Estudos de prevalência em saúde mental}

Os estudos de prevalência são os estudos observacionais mais amplamente difundidos e publicados em epidemiologia. Nesse tipo de delineamento, também conhecido como estudo transversal ou de cortetransversal, obtém-se a frequência de ocorrência dos eventos de saúde em uma população em um ponto específico no tempo (prevalência pontual) ou em determinado espaço de tempo (prevalência por período).

Na epidemiologia psiquiátrica, a medida de prevalência no período é a mais usada, em razão do curso episódico da maioria dos transtornos psiquiátricos (Fleming, Hsieh, 2002). Assim, ao contrário da prevalência pontual, a prevalência para períodos não considera apenas um ponto específico no tempo, mas abrange um período maior para seu cálculo e expressa a frequência de indivíduos que já apresentaram determinada doença no período considerado, na população estudada (Medronho et al. 2003).

Belfer (2008) aponta que, a partir do ano 2000, estudos que avaliam a prevalência de transtornos psiquiátricos na infância e adolescência começaram a ser realizados em países em desenvolvimento, incluindo o Brasil e outros países da América Latina. Tais estudos têm encontrado prevalências semelhantes às de países desenvolvidos, cujas estimativas sinalizam que uma entre quatro ou cinco crianças no mundo sofre de algum transtorno psiquiátrico (Patel et al. 2007).

Estudos epidemiológicos têm demonstrado diferenças de gênero na incidência, prevalência e curso de transtornos mentais e do comportamento. 
Os resultados indicam que as mulheres apresentam maiores taxas de prevalência de transtornos de ansiedade e do humor do que homens. Estes apresentam maior prevalência de transtornos associados ao uso de substâncias psicoativas, incluindo álcool; transtornos de personalidade antissocial e esquizotípica; transtornos do controle de impulsos; e transtorno de déficit de atenção com hiperatividade (TDAH) na infância e na vida adulta. Nos transtornos em que a prevalência é semelhante em homens e mulheres, observam-se diferenças na idade de início, perfil sintomatológico e resposta ao tratamento. Ainda têm sido identificados diferentes padrões de comorbidade psiquiátrica e psiquiátrica/física em homens e mulheres (Andrade et al., 2006).

\subsection{Prevalência dos transtornos psiquiátricos na adolescência}

Segundo Kielling et al. (2011), um transtorno psiquiátrico está presente em pelo menos uma de cada 10 crianças e adolescentes em todo 0 mundo, constituindo-se em importante causa de morbimortalidade. Entre jovens com idade de 10 a 24 anos, os transtornos psiquiátricos são responsáveis por $45 \%$ dos anos vividos com incapacidade (Gore et al. 2011). Enquanto os transtornos mentais e de uso de substâncias foram a principal causa de DALY no grupo etário entre zero e 24 anos dos países de alta renda, eles ocuparam o $7^{\circ}$ lugar em países de baixa e média renda devido à maior mortalidade atribuível a doenças infecciosas nestes países (Erskine et al. 2015). 
Dados epidemiológicos globais atuais da OMS reportam que cerca de $20 \%$ das crianças e adolescentes com até 16 anos de idade sofrem de uma doença mental incapacitante. Essa prevalência varia de 12,8\% na Índia (indivíduos com idade entre um e 16 anos) a 22,5\% na Suíça (indivíduos com idade entre um e 15 anos). Uma ressalva deve ser feita a essas estimativas elevadas de morbidade entre crianças e adolescentes: a infância e a adolescência são fases de desenvolvimento em que ocorrem constantes mudanças no comportamento, o que dificulta traçar limites claros entre fenômenos que fazem parte do desenvolvimento normal e outros que são anormais ao desenvolvimento.

Uma recente revisão sistemática e meta-análise da prevalência dos transtornos psiquiátricos em adolescentes, que incluiu 27 países, relatou uma prevalência mundial de transtornos psiquiátricos de 13,4\% (IC95\% 11,3-15,9). Os autores observaram bastante semelhança na frequência de transtornos psiquiátricos entre os diferentes países e culturas, uma vez que os fatores metodológicos dos estudos foram adequadamente controlados (Polanczyk et al. 2015). Estudos realizados no Brasil relataram uma prevalência de transtornos psiquiátricos na adolescência entre $7 \%$ e $20 \%$, dependendo da região investigada, da exposição a fatores de risco e da metodologia empregada nos estudos (Kielling et al. 2011).

Outros estudos realizados em países de rendas baixa e média e que aplicaram o instrumento DAWBA apresentaram prevalências semelhantes, embora usando faixas etárias mais amplas. Em estudo de prevalência em duas fases aplicado a amostras aleatórias de crianças de cinco a 10 anos 
( $n=922)$, provenientes de três áreas contrastantes de Bangladesh e da Índia, foi observada uma prevalência global de algum transtorno psiquiátrico em $15,2 \%$ da amostra $(15,4 \%$ na zona rural; $10 \%$ em uma área urbana moderadamente próspera; e 19,5\% em favela urbana) (Mullick et al. 2015). Na Rússia, em uma amostra de 448 adolescentes com idade de sete a 14 anos, a frequência observada de transtornos psiquiátricos usando o instrumento DAWBA foi de $15,3 \%$-- $70 \%$ maior do que a prevalência observada pelos mesmos autores em crianças e adolescentes da GrãBretanha, em estudo prévio (Goodman et al. 2005; Ford et al. 2003).

Um estudo de amostra populacional de crianças e adolescentes na Inglaterra, Escócia e País de Gales, com objetivo de investigar a prevalência de transtorno mental e seu impacto na saúde mental das crianças e adolescentes, demonstrou que $10 \%$ dos indivíduos com idade entre cinco e 15 anos apresentavam algum tipo de transtorno mental. A prevalência foi maior nos meninos, em indivíduos de cor da pele preta, em famílias monoparentais, com cinco filhos ou mais, com baixa escolaridade dos pais e em aqueles de família de baixa renda (Ford et al. 2003).

Merikangas et al. (2010) avaliaram a presença de transtorno mental em 10.123 adolescentes de 13 a 18 anos nos Estados Unidos. Encontraram uma prevalência de transtorno mental de $22,2 \%$ na população estudada, sendo $11,2 \%$ transtornos de humor; $8,3 \%$ transtornos de ansiedade; e $9,6 \%$ transtornos de comportamento. Considerando a idade de manifestação dos primeiros sintomas, observaram que os transtornos de ansiedade iniciavam em média aos seis anos; os transtornos de comportamento, aos 11 anos; os 
transtornos de humor, aos 13 anos; e os transtornos por uso de substâncias, aos 15 anos.

No Brasil, Fleitlich-Bilyk, Goodman (2004) conduziram um estudo com o objetivo de avaliar a saúde mental de uma amostra probabilística de 1.251 escolares das oito séries do ensino fundamental do município de Taubaté, no interior de São Paulo. Os pais/responsáveis, os adolescentes de 11 a 14 anos e os professores responderam ao instrumento DAWBA. Os autores observaram que 12,7\% (IC95\% 9,8-15,5) dos escolares apresentavam algum transtorno mental, aproximadamente uma a cada oito crianças.

Ainda na cidade de Taubaté-SP, estudo que avaliou 454 crianças e adolescentes, na faixa etária de sete a 11 anos de escolas públicas e particulares do município, relatou que $35,2 \%$ dos indivíduos foram considerados positivos para algum transtorno psiquiátrico $(22,7 \%$ alcançaram nível clínico e $12,5 \%$ nível limítrofe na escala total de dificuldades do SDQ) (Vitolo et al. 2005).

Paula et al., na cidade de Embu das Artes-SP, avaliaram 479 crianças e adolescentes de seis a 17 anos, utilizando o instrumento CBCL, e observaram uma prevalência de qualquer problema mental de 7,3\% (IC95\% 5,0-9,6) (Bordin, Paula, 2007).

Fleitlich, Goodman (2001) estimaram a prevalência de problemas de saúde mental em crianças e adolescentes na faixa etária de sete a 14 anos, residentes no município de Campos do Jordão-SP, provenientes de três áreas distintas: uma favela com casas improvisadas, sem saneamento e instaladas ilegalmente; uma comunidade urbana bem estabelecida; e uma 
comunidade rural. Nestas três áreas, os indivíduos participantes do estudo foram identificados a partir das escolas. Foram avaliados 898 estudantes, sendo 488 da favela, 346 da comunidade urbana e 64 da comunidade rural. No total, $15 \%$ dos estudantes apresentaram problemas de saúde mental, segundo o SDQ, sendo observada maior taxa de problemas entre crianças e adolescentes da favela (22\%) do que entre crianças e adolescentes da comunidade urbana (12\%) e da rural (12\%).

Recentemente, um estudo transversal, nacional de base escolar, realizado em municípios brasileiros com mais de 100 mil habitantes, onde foram avaliados 74.589 adolescentes participantes do Estudo de Riscos Cardiovasculares em Adolescentes (ERICA) relatou, na faixa etária entre 12 e 14 anos, uma prevalência de transtornos mentais comuns (avaliados pelo General Health Questionnaire - GHQ-12) de 26,7\% (Lopes et al., 2016).

Em estudo de coorte transversal, Goodman et al. (2005) avaliaram a saúde mental de crianças e adolescentes com idade entre cinco e 14 anos, na llha de Maré-BA, a partir de uma amostra probabilística de 519 participantes, sendo 430 em idade escolar na faixa etária dos sete aos 14 anos. Os autores verificaram, a partir do SDQ, que as taxas de prevalência para o total de problemas de saúde mental variaram de acordo com o tipo de informante (pais, professores, próprio adolescente). As taxas foram de $15,1 \%$, segundo os pais; $10,3 \%$, segundo os professores; e $13,1 \%$, segundo os adolescentes. Essa diferença entre a frequência de transtornos psiquiátricos observada conforme diferentes informantes também foi relatada por Cury et al. (2003). Os investigadores realizaram um estudo transversal 
em uma escola da cidade de Ribeirão Preto-SP, com o objetivo de verificar a prevalência de transtornos psiquiátricos em 108 crianças e adolescentes na faixa etária entre seis e 11 anos. Os pais e professores destas crianças e adolescentes responderam ao SDQ, o que resultou em taxas de prevalência de transtornos psiquiátricos diferentes. A prevalência de transtornos psiquiátricos foi de $18,7 \%$ na avaliação dos pais e de $8,3 \%$ na avaliação de professores.

Em Pelotas-RS, estudo conduzido por Anselmi et al. (2010), com 4.423 adolescentes de 11 anos pertencentes à Coorte de Nascimentos de 1993, utilizando o instrumento SDQ, observou uma prevalência de qualquer transtorno mental avaliada pelo DAWBA, de 10,8\% (IC95\% 7,1-14,5).

Petresco et al. (2014) avaliaram a prevalência de transtorno psiquiátrico em 3.585 crianças de seis anos, na Coorte de Nascimentos de 2004 de Pelotas-RS, na mesma população de estudo do presente projeto de pesquisa. A avaliação dos transtornos psiquiátricos foi realizada com 0 instrumento DAWBA e as classificações seguiram o DSM-IV e a CID-10. Os autores encontraram no estudo uma prevalência de transtorno psiquiátrico de aproximadamente $13 \%$, que variou em relação ao sexo e à renda familiar. Nos meninos a prevalência foi de $14,7 \%$ e nas meninas, de $11,7 \%$. Nas crianças de famílias de baixa renda, a prevalência de transtorno mental foi maior do que nas crianças de famílias de renda alta $(14,0 \%$ vs. $8,0 \%)$. 0 transtorno de ansiedade foi o mais prevalente $(8,8 \%)$, seguido pelas fobias específicas $(5,4 \%)$ e transtorno de ansiedade de separação $(3,2 \%)$. 


\subsection{Continuidade dos transtornos psiquiátricos na adolescência}

Segundo Rutter (1984) a continuidade da psicopatologia é definida como um padrão preditivo de associações entre comportamentos prévios e algum tipo de desfecho posterior na vida do indivíduo. Diversas pesquisas confirmam que existe uma continuidade entre problemas de saúde mental na infância, na adolescência e na vida adulta, sendo que a maioria dos indivíduos diagnosticados com transtornos psiquiátricos na vida adulta apresentou algum transtorno psiquiátrico na infância ou adolescência (Mathiesen, Sanson 2000; Rutter et al. 2006). Outros investigadores também observaram que os transtornos de internalização, como a ansiedade e depressão, e os transtornos de externalização, como os transtornos de conduta e oposição, com início na adolescência, estão associados a uma série de doenças psiquiátricas na fase adulta (Kim-Cohen et al. 2003).

Diversos estudos demonstraram que na trajetória do desenvolvimento dos transtornos mentais, tanto uma continuidade homotípica - por exemplo, quando um transtorno ansioso na infância precede um transtorno ansioso na vida adulta - quanto uma continuidade heterotípica, quando o adolescente apresenta diferentes transtornos ao longo dos anos ou o mesmo transtorno se manifesta de diferentes maneiras ao longo do tempo - por exemplo, um transtorno de conduta e oposição que precede o uso de substâncias (Costello et al. 2003) -, são uma constante na evolução dos transtornos mentais (Ferdinand, Verhulst, 1995, Hofstra et al. 2000, Kim-Cohen et al. 2003). Ou seja, os padrões de sintomas podem mudar ao longo da vida, 
refletindo os diferentes aspectos da duração e do tipo de experiências vividas pelo indivíduo nas diferentes idades (Rutter et al. 2006).

Kim-Cohen et al. (2003), ao estudarem os dados de 1.037 participantes de uma coorte de nascimentos com faixa etária entre 11 e 26 anos, relataram que $80 \%$ dos jovens que apresentaram algum transtorno psiquiátrico aos 11 anos também desenvolveram algum transtorno psiquiátrico aos 26 anos, não necessariamente o mesmo transtorno.

Costello et al. (2003) realizaram um estudo longitudinal com três coortes de crianças e adolescentes com idades de nove, 11 e 13 anos, em 11 municípios do Oeste da Carolina do Norte (Estados Unidos) e observaram que houve continuidade dos transtornos psiquiátricos identificados na coorte. Os autores relataram que a continuidade homotípica foi maior do que a heterotípica, sendo mais frequente nas meninas do que nos meninos.

Outros investigadores também observaram que a continuidade homotípica, como algum transtorno de conduta e oposição e TDAH, foi mais frequente do que a continuidade heterotípica na faixa etária entre os 12 e 19 anos (Costello et al. 2011).

Uma coorte de nascimento de mais de mil indivíduos na Nova Zelândia estudou a associação entre comportamentos ansiosos nos primeiros anos de vida e o desenvolvimento de transtornos de internalização na faixa etária de 16 a 21 anos. Os autores concluíram que o comportamento ansioso na infância esteve associado a elevadas taxas de transtornos de ansiedade e depressão durante a adolescência e a idade 
adulta (Goodwin et al. 2004), o que posteriormente também foi relatado por outros autores (Rutter et al. 2006).

Estudo recente realizado em Londres com 8.099 crianças e adolescentes, com idade entre nove e 11 anos, de 73 escolas primárias, avaliou a continuidade dos problemas de internalização e de externalização nessa população. Os autores concluíram que houve continuidade dos transtornos de internalização e de externalização desde a infância até o início da adolescência (Lancefield et al. 2016).

Outro estudo realizado recentemente em uma amostra comunitária da Noruega, com 1.042 crianças e adolescentes entre quatro e 10 anos, concluiu que houve continuidade homotípica do transtorno de conduta e oposição nesta faixa etária (Husby, Wichstrom, 2016).

Estudo realizado com 462 crianças na cidade de Nova lorque avaliou a continuidade dos transtornos psiquiátricos em uma grande amostra comunitária de pré-escolares (três anos) até o início da idade escolar (seis anos). Os autores relataram que houve continuidade homotípica para algum transtorno de ansiedade, TDAH e transtorno de conduta e oposição e continuidade heterotípica para depressão e algum transtorno de ansiedade (Bufferd et al. 2012).

Estudo realizado na China com 399 crianças, acompanhadas da infância até o início da idade adulta, relatou que 46,37\% dos participantes ainda atendiam aos critérios de TDAH na adolescência e 72,69\% continuavam com persistência total ou parcial do TDAH na idade adulta (Gao et al. 2015). 
No Brasil estudo com 601 crianças acompanhadas aos quatro e aos 12 anos, pertencentes ao estudo de Coorte de Nascimento de 1993 de Pelotas-RS, mostrou que $31 \%$ do total de crianças com problemas comportamentais e/ou emocionais aos quatro anos continuaram com transtornos mentais aos 12 anos (Anselmi et al. 2008). Os autores também concluíram que houve uma maior continuidade homotípica, ao longo dos anos, dos transtornos de externalização.

\subsection{Fatores de risco dos transtornos psiquiátricos na adolescência}

Fatores de risco são variáveis ambientais, comportamentais ou biológicas, estabelecidas em uma sequência temporal em relação à doença. Quando presentes, os fatores de risco aumentam a probabilidade da ocorrência de determinado desfecho em uma pessoa ou grupo em comparação com indivíduos não expostos a eles; quando ausentes, a reduzem (Walker et al. 2007; Pesce et al. 2004).

Os fatores de proteção são características individuais (recursos pessoais) e/ ou contextuais (recursos ambientais) que fortalecem e dão suporte ao indivíduo no enfrentamento de diferentes eventos de vida. São exemplos de fatores de proteção: autonomia, autoestima, bem-estar subjetivo, competência emocional, afetos positivos, apoio social, coesão familiar, entre outros (Poletto, Koller, 2008). Noronha et al. (2009) apontam que os fatores de proteção podem reduzir o impacto dos riscos ou de reações negativas. 
A saúde mental dos indivíduos é influenciada por características individuais, comportamentais, socioeconômicas e ambientais. Baixa autoestima, dificuldade na escola, baixa renda e pobreza, exposição à violência e abuso, desigualdades sociais e de gênero são considerados fatores de risco para problemas de saúde mental. Por outro lado, confiança e autoestima elevada, apoio social da família e amigos, uma boa interação familiar, segurança econômica, satisfação e sucesso no trabalho, acesso a serviços básicos de saúde, entre outros, são fatores protetores (Noronha et al. 2009).

Os transtornos psiquiátricos de início precoce estão frequentemente associados a fatores de risco mais graves (Beddington et al. 2008; Moffit et al. 2002) e a piores prognósticos na vida adulta (Beddington et al. 2008). Existem poucas evidências de associações específicas entre um fator de risco particular e um determinado transtorno psiquiátrico. Possivelmente, a combinação, sequência e inter-relação de fatores de risco individuais, familiares, genéticos e ambientais sejam a explicação mais razoável para o desenvolvimento dos transtornos mentais (Goodman et al. 2005; Ford et al. 2004).

Os riscos em muitos casos ocorrem associadamente (em cadeia), expondo o indivíduo a mais situações adversas, que vão se agravando com a ausência de tratamento ou de fatores de proteção. Tais situações adversas constituem-se como mecanismos de risco e potencializam a probabilidade de desfechos negativos e de problemas de saúde mental. Os variados fatores de risco conhecidos para transtornos mentais comumente ocorrem 
simultaneamente nos mesmos indivíduos e nas mesmas famílias (Bordin, Paula, 2007).

Como as adversidades não costumam estar isoladas, uma vez que se inserem em um contexto social que envolve fatores políticos, socioeconômicos, ambientais, culturais, familiares e genéticos, seria errôneo pensar que somente um fator de risco poderia ocasionar problemas a uma determinada criança ou adolescente (Bordin et al. 2007; Halpern, Figueiras, 2004; Sapienza, Pedromônico, 2005). A compreensão de como estes fatores interagem em diversos pontos, durante o desenvolvimento, pode ajudar a identificar como e quando intervir para que as crianças e adolescentes possam atingir seu pleno potencial (Walker et al. 2007). Existem evidências de que os fatores de risco são bastante similares em diferentes culturas. $O$ que parece variar são a intensidade e a magnitude destes fatores (Canino, Alegria, 2008). Logo, apesar de existirem semelhanças entre os fatores de risco dos países desenvolvidos e aqueles dos países em desenvolvimento (Hackett et al. 1999), a população dos países em desenvolvimento encontrase mais exposta a potenciais fatores de risco como, por exemplo, a pobreza (Duarte et al. 2003).

A Figura 1 é uma adaptação do modelo de Bronfenbrenner (1979). Este modelo permite o entendimento de como diferentes contextos podem atuar tanto como fatores que aumentam o risco de transtornos psiquiátricos, quanto como fatores protetores que minimizam seus impactos. 


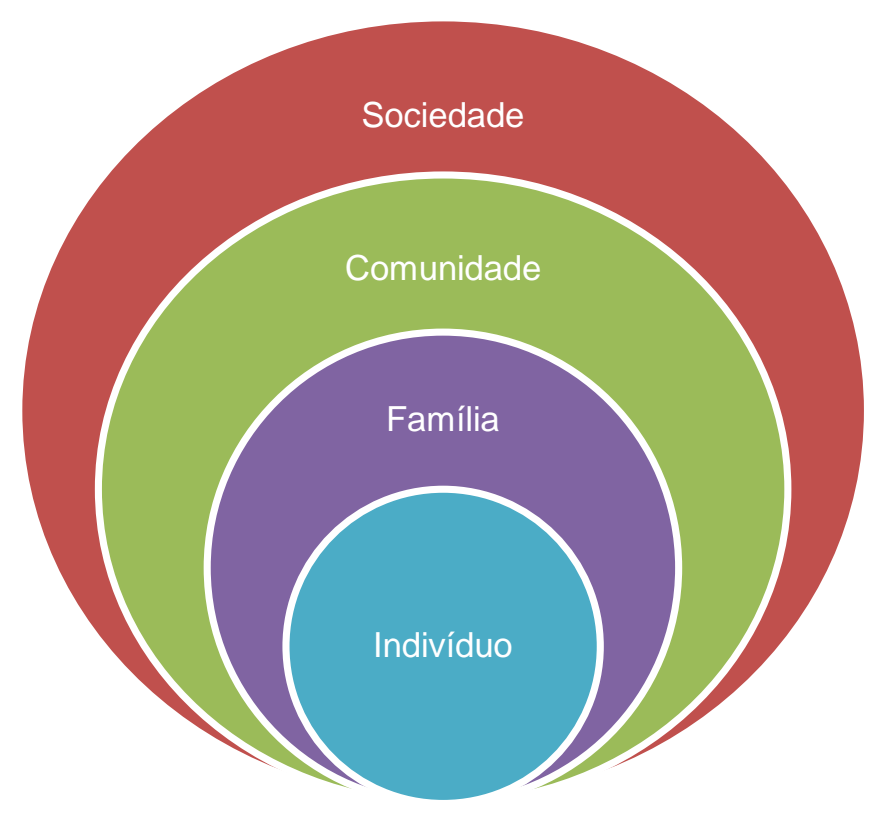

Figura 1 - Modelo para entender os fatores de risco e de proteção dos transtornos psiquiátricos

FONTE: Bronfenbrenner (1979), adaptado pelo autor.

Apesar de diversos fatores de risco contribuírem na etiologia dos transtornos mentais, as respostas dos indivíduos, quando expostos a tais fatores, são bastante variadas. Essa heterogeneidade de respostas, que influencia na resiliência ou na vulnerabilidade dos indivíduos, geralmente está associada a características geneticamente determinadas, como o temperamento do indivíduo (Moffitt, Caspi, 2006). A resiliência apresenta diversas definições que vão desde um conjunto de competências e habilidades individuais - resultado de traços de personalidade e invulnerabilidade, influências ambientais, manifestação de competências diante de circunstâncias adversas - até o resultado do equilíbrio entre fatores protetores e de risco, tanto individuais quanto sociais (Souza, Cerveny, 2006). Os autores destacam que o conceito de resiliência é um processo de construção, compartilhamento e ressignificação de 
experiências, envolvendo a resiliência familiar e comunitária e não apenas a individual, já que o conceito de resiliência está impregnado nos contextos socio-histórico e cultural.

A Epidemiologia do Ciclo Vital (ECV) estuda os processos biológicos, comportamentais e psicossociais que ocorrem ao longo da vida dos indivíduos e que ligam a saúde do adulto e o risco de adoecer a exposições físicas ou sociais que aconteceram durante a gravidez, infância, adolescência, nas etapas precoces da vida adulta ou através de gerações (Ben-Shlomo, Kuh 2002).

Os fatores que afetam o crescimento na vida intrauterina ou na infância precoce podem influenciar o desenvolvimento de problemas de saúde mental a longo prazo. O tabagismo durante a gestação, por exemplo, tem implicações que vão além dos prejuízos à saúde materna. A exposição pré e perinatal à nicotina tem sido relacionada a alterações da cognição, além de causar importantes alterações no desenvolvimento do sistema nervoso fetal (Lieberman et al. 1994). O fumo materno durante a gestação leva a mudanças sutis no desenvolvimento neurológico e no comportamento das crianças, tais como uma redução geral na capacidade intelectual e prédisposição ao desenvolvimento do TDHA durante a infância e adolescência (Weissman et al. 1999).

No estudo de Brion et al. (2010), comparando a Coorte Inglesa de Avon Longitudinal Study of Parents and Children (ALSPAC) com a Coorte de Nascimentos de 1993 de Pelotas-RS, os autores observaram nas análises ajustadas para psicopatologia parental que o fumo materno durante a 
gravidez esteve associado a problemas de externalização e de conduta nas crianças de ambas as coortes. O risco de problemas de conduta nos filhos de mães fumantes foi maior do que nos filhos de mães não fumantes (OR=1,24 IC95\% 1,07-1,46, na coorte de ALSPAC; e OR=1,82 IC95\% 1,192,78, na coorte de Pelotas).

O uso de álcool durante a gestação pode causar danos no desenvolvimento da criança, sendo que os efeitos adversos da exposição pré-natal ao álcool podem variar. Os indivíduos afetados podem apresentar dificuldades relacionadas à atenção e à concentração e alterações do comportamento, como hiperatividade, impulsividade e problemas de conduta (Huizink et al. 2006; Fagerlunf et al. 2011; Riley et al. 2011; Jacobson et al. 2011). Isso, além da síndrome alcoólica fetal, que é considerada a principal causa de déficit no desenvolvimento com causa conhecida (Paley 2009). No nascimento há sinais de disfunção do sistema nervoso central nos bebês de mães que ingeriram grande quantidade de álcool durante a gestação. Há relatos de intoxicação, dependência, delirium, demência persistente, perturbação psicótica, perturbação do humor, perturbação da ansiedade, disfunção sexual e disfunção do sono (McGee, Riley 2007; APA, 2014). Atualmente, está bem estabelecido que crianças de pais alcoolistas são mais vulneráveis a problemas de comportamento, distúrbios psiquiátricos, abuso de substâncias (incluindo início precoce de uso do álcool) e a problemas inerentes ao uso persistente de álcool (Chassin, Fora et al. 2004). Nessa perspectiva, múltiplos fatores - biológico, psicológico e social - e as influências originadas no ambiente familiar, principalmente as influências 
parentais, são relevantes para o desenvolvimento do abuso e da dependência de álcool em seus descendentes (Jacob et al. 2009).

Estudo realizado com 649 adolescentes entre 12 e 19 anos verificou o abuso de álcool em filhos de alcoolistas e constatou que um em cada quatro adolescentes que se identificaram como abusadores de álcool eram filhos de alcoolistas (Rodney et al. 1996). Outro estudo mostrou os altos índices de reprodução do alcoolismo entre jovens adultos, apontando que um em cada três jovens adultos dependentes do álcool tem histórico de alcoolismo na família (Souza et al. 2005).

A prematuridade é um indicador da saúde do recém-nascido que também reflete condições da vida intrauterina. Um estudo de revisão sobre o efeito do nascimento prematuro no desenvolvimento e na qualidade de vida de crianças mostrou que as variáveis peso ao nascimento e idade gestacional foram preditoras de problemas de comportamento internalizante, como os transtornos de ansiedade e depressão nas fases pré-escolar e escolar (Fallang et al. 2005; Vieira, Linhares, 2012). Outros autores reportaram que crianças nascidas pré-termo apresentaram também mais problemas de comportamento do tipo externalizante na infância e adolescência em comparação com crianças nascidas a termo (WhitesideMansell et al. 2009), além do risco de desenvolver transtornos do comportamento, esquizofrenia, hiperatividade e déficits de atenção, problemas de comportamento, sintomas semelhantes a psicoses, transtornos afetivos e suicídio na adolescência ou na vida adulta (Breslau, Chilcoat, 2000; Martinéz-Cruz et al. 2006; Wiles et al. 2006; Carvalho et al. 
2001). As crianças com baixo peso ao nascimento têm sido consideradas mais vulneráveis aos déficits cognitivos (Martinéz-Cruz et al. 2006; Gale et al. 2004; Patton et al. 2004), às dificuldades comportamentais (Kelly et al. 2001; Linhares et al. 2005) e às desordens emocionais, dentre elas a depressão infantil (Hayes, Sharef, 2009), além de apresentarem 40 vezes mais risco de sequelas no seu desenvolvimento físico e intelectual (Saigal et al. 2003).

A psicopatologia nos pais é um forte preditor dos transtornos mentais nos filhos. A presença de história de depressão, ansiedade materna, exposição a fatores estressores, como morte de um familiar durante a gestação, e problemas psicológicos crônico na mãe mostrou-se associada a um maior risco de transtorno mental nos filhos ao longo da vida. Cerca de $40 \%$ dos filhos de pais depressivos apresentam um ou mais transtornos mentais (Piche et al. 2011; Khashan et al. 2008; Ensminger et al. 2003).

Estudo realizado com indivíduos pertencentes à Coorte de Nascimentos de 1993 de Pelotas-RS relatou que a presença de transtorno psiquiátrico materno foi um fator fortemente associado aos problemas emocionais e de comportamento em crianças aos quatro anos (Anselmi et al. 2004). Em uma avaliação do perfil comportamental e da competência social de crianças e adolescentes de seis a 18 anos, os filhos de mães com diagnóstico de esquizofrenia apresentaram maior proporção de problemas de internalização, como transtornos de ansiedade e depressão, quando comparados aos filhos de mulheres sem transtornos mentais (Gutt, 2005). 
O sexo é um dos fatores de risco que tem sido bastante investigado em estudos epidemiológicos. Vários estudos sugerem que os meninos são mais propensos a desenvolver algum transtorno mental do que as meninas (Ford et al. 2004). Os transtornos com início precoce, em geral, são mais prevalentes nos meninos, enquanto que aqueles que se iniciam na adolescência são mais prevalentes nas meninas (Rutter et al. 2003). Essas diferenças variam entre os grupos etários. $\mathrm{Na}$ infância, a maioria dos estudos relata uma maior prevalência de transtornos de conduta, por exemplo, com comportamentos agressivos e antissociais, entre meninos do que entre meninas (Polanczyk et al. 2007; Moffitt et al. 2008). Durante a adolescência, as meninas têm uma prevalência maior de depressão e de transtornos alimentares e se envolvem mais na ideação suicida e tentativas de suicídio do que os meninos. Já os meninos experimentam mais problemas com raiva, envolvem-se em comportamentos de alto risco e cometem suicídio com mais frequência do que as meninas (Philipp et al. 2014, Pacheco et al. 2005). Estudo realizado por Rescorla et al. (2007), com amostras populacionais de adolescentes de 24 países, demonstrou importantes diferenças entre os sexos na prevalência de problemas de internalização e externalização. No estudo, os meninos apresentaram mais problemas de externalização e as meninas mais problemas de internalização. Estudo brasileiro recente, realizado por Petresco et al. (2014) com crianças aos seis anos de idade, na Coorte de Nascimentos de 2004 de Pelotas-RS (mesma população do presente projeto), mostrou uma maior 
prevalência de transtorno psiquiátrico nos meninos em comparação com as meninas $(14,7 \%$ e $11,7 \%$, respectivamente; $p=0.009)$.

Sobre os fatores de risco ambientais associados a transtornos mentais, o nível socioeconômico é o mais robusto dos achados em estudos epidemiológicos, existindo uma associação inversa: quanto menor o nível socioeconômico, maior a ocorrência de transtornos mentais (APA, 2014; Piche et al. 2011; Rutter et al. 2003; Costello et al. 1996). Edward Jarvis relatou, já em 1855, que os transtornos psiquiátricos ocorriam 64 vezes mais frequentemente em pobres do que em ricos (Jarvis E, 1971).

Existem duas distintas teorias que tentam explicar esse fenômeno: a causalidade social e a seleção social. A primeira sugere que o estresse associado à baixa posição socioeconômica (PSE) causaria transtorno mental. Já a segunda teoria propõe que a associação se deve à queda na posição social, produzida pelo transtorno mental (Dohrenwend et al. 1992).

A combinação de baixa renda, analfabetismo, desemprego, más condições de moradia e acesso limitado à saúde e à educação aumenta o risco de transtornos mentais (Beddington et al., 2008, Bordin et al. 2007; Benvegnu et al., 2005, Vitolo et al., 2005, Fleitlich; Goodman, 2001). Os autores salientam a associação da pobreza com inúmeras condições adversas e com uma maior exposição a fatores de estresse. Uma revisão sistemática que avaliou os efeitos da PSE na ocorrência de transtornos mentais em crianças e adolescentes observou que a baixa renda familiar e a menor educação dos pais estiveram associadas a uma maior frequência de transtornos mentais em crianças e adolescentes, quando comparados 
àqueles que eram filhos de pais com maior renda e/ou educação (Reiss et al. 2013). Em estudo nacional, com dados da mesma coorte utilizados nesta tese, foi observado que crianças de famílias de baixa renda tiveram maior prevalência de algum transtorno mental do que crianças de famílias de alta renda ( $14 \%$ e $8 \%$, respectivamente; $p=0.001)$, achado este consistente com outros estudos (Van Oort FV et al. 2011; Costello et al. 2011).

A Figura 2 é uma adaptação de uma ilustração realizada por Patel et al. (2007) que representa o ciclo vicioso da relação entre a pobreza e os transtornos mentais. A baixa escolaridade e desigualdade social aumentam o risco de transtornos mentais, atingindo o poder econômico dos sujeitos, levando a privação econômica e impactando negativamente na escolaridade e aumentando a desigualdade social.

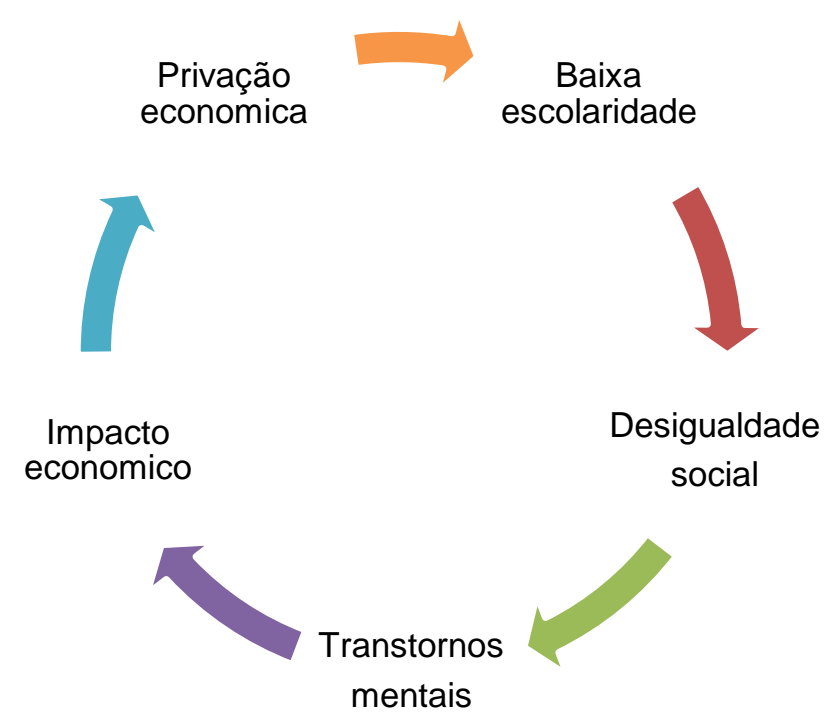

Figura 2 - O ciclo da pobreza e do transtorno mental FONTE: Patel et al. (2007), adaptado pelo autor. 
Para melhor compreensão do desenvolvimento dos fatores de risco durante o curso da vida, os principais fatores associados aos transtornos mentais estão apresentados na Figura 3 (adaptação do modelo de Beddington 2008). O modelo apresenta a relação de diferentes fatores de risco que podem agir em conjunto ou individualmente em diferentes etapas da vida do indivíduo. O modelo também permite identificar a relação entre os fatores e como esses fatores articulam-se entre si.

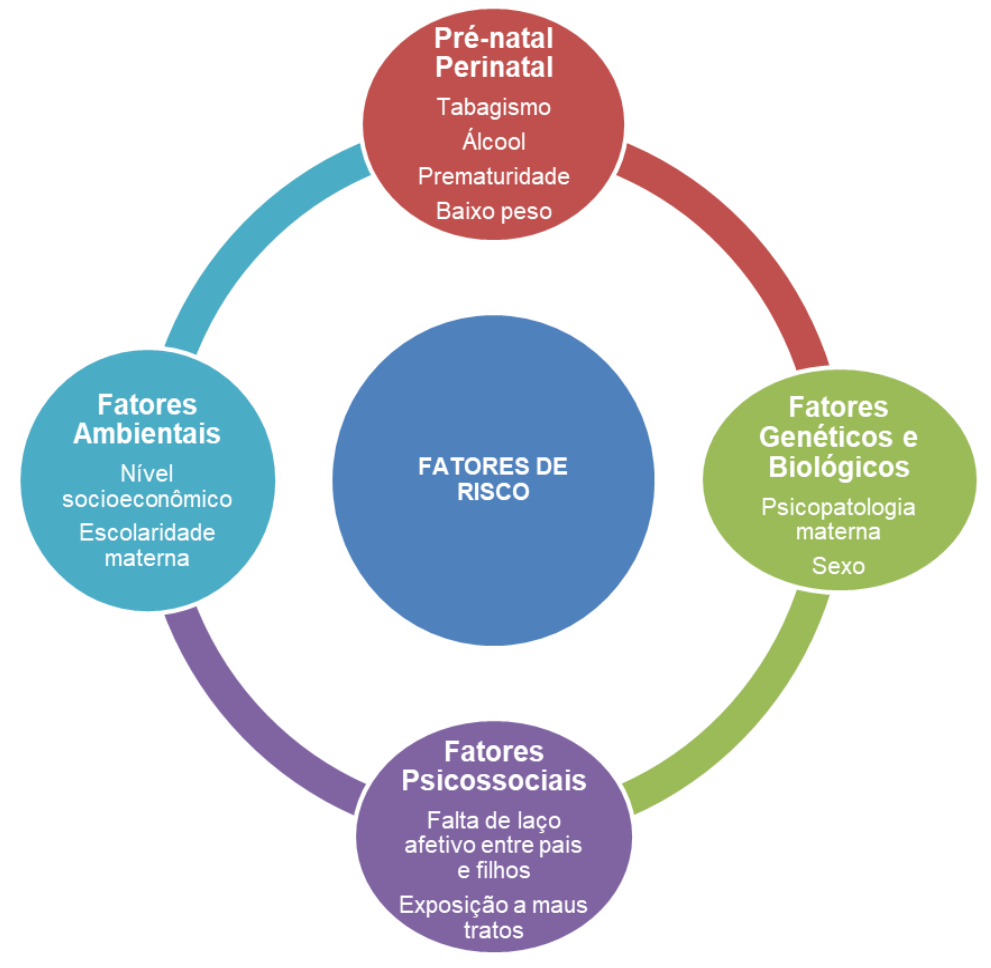

Figura 3 - Modelo teórico dos fatores de risco

FONTE: Beddington (2008), adaptado pelo autor. 
"O sucesso nasce do querer, da determinação e persistência em se chegar a um objetivo. Mesmo não atingindo o alvo, quem busca e vence obstáculos, no mínimo fará coisas admiráveis". 


\section{OBJETIVOS}

\subsection{Objetivo Geral}

Estudar a prevalência, continuidade e fatores de risco dos transtornos mentais no início da adolescência (11 anos) na Coorte de Nascimentos de 2004 do Município de Pelotas-RS, Brasil.

\subsection{Objetivos Específicos}

1. Descrever a prevalência total e específica dos transtornos psiquiátricos nos adolescentes aos 11 anos de idade.

2. Avaliar fatores de risco precoces dos transtornos psiquiátricos na adolescência.

3. Descrever a continuidade dos transtornos psiquiátricos entre os seis e 11 anos, agrupados em quatro grandes grupos de diagnóstico: transtornos de ansiedade, transtornos depressivos, TDAH e transtorno conduta e oposição. 
"Na vida, não vale tanto o que temos, nem tanto importa o que somos. Vale o que realizamos com aquilo que possuímos e, acima de tudo, importa o que fazemos de nós!" 


\section{METODOLOGIA}

Pelotas é um município da Região Sul do Brasil, localizada no Estado do Rio Grande do Sul (RS). Possui uma população de aproximadamente 328 mil habitantes, dos quais $93,3 \%$ vivem na zona urbana da cidade. Em 2000, o munícipio apresentava uma taxa de alfabetização de adultos de 95,7\%, maior do que a observada no Brasil (72\%), e seu Índice de Desenvolvimento Humano (IDH) médio era de 0,816, também maior do que o valor nacional $(0,699)$ (IBGE, 2011). A taxa de mortalidade infantil da cidade, no ano de 2012, era de 14,3 por mil nascidos vivos, um pouco abaixo da taxa de mortalidade no país (15,7 por mil nascidos vivos) (IBGE, 2012).

No ano de 2004, todas as crianças nascidas na zona urbana da cidade de Pelotas foram identificadas nos hospitais e suas mães convidadas a fazer parte de um estudo de coorte. No total, 4.231 crianças nascidas vivas das mães que aceitaram participar do estudo fizeram parte do grupo para posterior acompanhamento (recusas $<1 \%$ ). Mais detalhes da metodologia do estudo podem ser encontrados em publicações prévias (Santos et al. 2011; Santos et al. 2014).

Os integrantes da Coorte de Nascimentos de 2004 foram acompanhados em vários momentos. Nestes acompanhamentos, as mães ou responsáveis foram entrevistadas sobre o crescimento, desenvolvimento, tipo de alimentação e morbidade das crianças.

Desde 2004, foram realizados seis seguimentos (três, 12, 24, 48 meses, e seis e 11 anos). O Quadro 1 apresenta o número de crianças incluídas em cada acompanhamento e a taxa de acompanhamento em cada 
visita. O sexto acompanhamento da coorte de 2004 foi realizado entre maio e outubro de 2015, quando os indivíduos tinham em média (desvio padrão) $11,0(0,3)$ anos de idade (taxa de acompanhamento de $87 \%$ ).

Quadro 1 - Descrição das entrevistas realizadas e das perdas em cada acompanhamento realizado na Coorte de Nascimentos de 2004 de Pelotas-RS

\begin{tabular}{|llllllll|}
\hline & Perinatal & $\begin{array}{l}\mathbf{3} \\
\text { meses }\end{array}$ & $\begin{array}{l}\mathbf{1} \\
\text { ano }\end{array}$ & $\begin{array}{l}\mathbf{2} \\
\text { anos }\end{array}$ & $\begin{array}{l}\mathbf{4} \\
\text { anos }\end{array}$ & $\begin{array}{l}\mathbf{6} \\
\text { anos }\end{array}$ & $\begin{array}{l}\mathbf{1 1} \\
\text { anos }\end{array}$ \\
Entrevistas & 4.231 & 3.985 & 3.90 & 3.86 & 3.79 & 3.72 & 3.566 \\
realizadas & & & 7 & 9 & 9 & 1 & \\
Óbitos & - & 66 & 82 & 88 & 94 & 95 & 98 \\
Recusas(\%) & 32 & 26 & 26 & 40 & 51 & 27 & 68 \\
& $(0,8)$ & $(0,6)$ & $(0,6)$ & $(0,9)$ & $(1,2)$ & $(0,6)$ & $(1,6)$ \\
Perdas (\%) & - & 154 & 216 & 234 & 287 & 388 & 499 \\
& & $(3,6)$ & $(5,1)$ & $(5,5)$ & $(6,8)$ & $(8,0)$ & $(11,8)$ \\
Perdas + Recusas & 32 & 180 & 242 & 274 & 338 & 415 & 567 \\
(\%) & $(0,8)$ & $(4,3)$ & $(5,7)$ & $(6,5)$ & $(8,0)$ & $(9,8)$ & $(13,4)$ \\
Taxa de & & & & & & & \\
acompanhamento & 99,2 & 95,7 & 94,3 & 93,5 & 92,0 & 90,2 & 86,6 \\
\hline
\end{tabular}

\subsection{População alvo}

A população alvo do estudo foram 3.566 adolescentes (das 4.231 crianças captadas no nascimento, 98 delas morreram até os 11 anos).

\subsection{Critérios de inclusão e de exclusão}

Neste estudo foram incluídos todos os adolescentes que tinham informações completas para o questionário sobre saúde mental - DAWBA respondido pelas mães ou responsáveis. 


\subsection{Definição e operacionalização do desfecho do estudo}

Os transtornos psiquiátricos dos adolescentes foram avaliados usando o instrumento DAWBA (Goodman, 2000) aplicado às mães por psicólogas treinadas. $O$ instrumento foi traduzido para $\mathrm{o}$ português $\mathrm{e}$ validado no Brasil seguindo cuidadosa adaptação cultural (Fleitlich-Bilyk, Goodman, 2004). O DAWBA contém uma parte estruturada e uma parte aberta e permite fazer diagnósticos psiquiátricos segundo as classificações diagnósticas do DSM-IV, DSM-5 e da CID-10, em crianças e adolescentes de 5 a 17 anos de idade.

Um algoritmo computadorizado possibilita a identificação da probabilidade de cada indivíduo apresentar algum transtorno psiquiátrico. No entanto, o autor do instrumento sugere que um profissional da área de saúde mental treinado (rater) avalie cada questionário aplicado para determinar a presença ou ausência de transtornos psiquiátricos. Para cada transtorno psiquiátrico, o entrevistador avalia cada um dos sintomas e outros critérios necessários para o diagnóstico de acordo com o DSM-IV, o DSM-5 e a CID10.

Foram coletadas informações das seguintes categorias nosológicas: transtorno de ansiedade de separação; fobia específica; fobia social; transtorno de ansiedade generalizada; transtorno de estresse póstraumático; transtorno do pânico e agorafobia; transtorno obsessivocompulsivo; transtorno depressivo, transtorno bipolar, TDAH; transtorno oposição desafiante; transtorno de conduta; transtornos do espectro autista; transtornos alimentares; transtorno de tique. Além disso, foram incluídos três 
novos diagnósticos presentes no DSM-5: o transtorno dismórfico corporal (Body Dysmorphic Disorder - BDD), o transtorno de conduta/oposição da desregulação do humor (DMDD) e o transtorno de compulsão alimentar (Binge Eating Disorder - BED).

Os diagnósticos do DAWBA foram descritos de forma dicotômica (sim/ não) respeitando-se estritamente os critérios diagnósticos definidos pelas classificações diagnósticas já mencionadas. A avaliação clínica do total da amostra foi realizada por um psicólogo; um segundo avaliador clínico independente avaliou $10 \%$ da amostra em estudo. Ambos foram treinados e padronizados por uma psiquiatra infantil que traduziu e validou o DAWBA no Brasil (Fleitlich-Bilyk, Goodman, 2004). A concordância entre os raters foi de $91,2 \%$ para a presença de algum transtorno psiquiátrico; $75,9 \%$ para algum transtorno de ansiedade; 73,5\% para algum transtorno depressivo; $72,7 \%$ para algum TDAH; 72,9\% para algum transtorno de conduta; 85,6\% para algum transtorno do espectro autista; $59,5 \%$ para algum transtorno alimentar; e 52,4\% para algum transtorno de tique.

Antes de aplicar o DAWBA, foi aplicado o instrumento SDQ junto a todas as mães. O SDQ é um questionário de triagem, composto por 25 questões, divididas em cinco subescalas, com cinco perguntas cada uma, gerando escores de problemas emocionais, de conduta, atenção e hiperatividade, relação com pares e comportamento pró-social, além do escore total. A versão extensiva do SDQ é formada por mais cinco perguntas que avaliam o impacto dos sintomas na vida das crianças e adolescentes. 


\subsection{Processamento dos dados}

Todas as entrevistas foram digitadas diretamente no programa on-line do DAWBA em tempo real. Posteriormente, o supervisor avaliou as entrevistas realizadas e conferiu os diagnósticos de transtornos mentais efetuados pelo programa estatístico do DAWBA.

\subsection{Análise dos dados}

As análises descritivas incluíram o cálculo de distribuições de frequência para variáveis dicotômicas, com os respectivos intervalos de confiança. Os testes estatísticos foram baseados no teste de qui-quadrado. As análises de prevalência de transtornos psiquiátricos foram realizadas para todos os indivíduos e separadamente para meninos e meninas. Utilizamos o Programa Estatístico Stata, versão 14, para realizar as análises estatísticas. 
"Só existem dois dias do ano em que você não pode fazer nada: um se chama ontem e outro amanhã".

Dalai Lama 


\section{PARTICIPAÇÃO NO TRABALHO DE CAMPO}

Após o ingresso no doutorado, realizei um estágio de quatro meses na cidade de Pelotas-RS, no Centro de Pesquisas Epidemiológicas da Universidade Federal de Pelotas (UFPel). Nesse período, participei das atividades realizadas pela equipe da coorte, colaborando com 0 desenvolvimento das atividades propostas para o acompanhamento aos 11 anos de idade da Coorte de Nascimentos de 2004 de Pelotas-RS.

No estágio, participei de todas as atividades, desde aquelas desenvolvidas antes do campo, como organização e planejamento, localização dos participantes, recrutamento de pessoal, treinamentos etc., até as atividades realizadas durante o trabalho de campo propriamente dito, como busca de participantes e entrevistas domiciliares. Participei da capacitação das entrevistadoras psicólogas para aplicação do instrumento DAWBA, auxiliando nas atividades da capacitação e na avaliação posterior das psicólogas.

A equipe da coorte de 2004, composta de pesquisadores, colaboradores e doutorandos - se reunia periodicamente para tratar das tarefas, responsabilidades e atualizações, antes e ao longo do trabalho de campo. Participei de todas as reuniões, contribuindo com a pauta e organizando relatórios. Também tive a oportunidade de apresentar os resultados da busca ativa realizada para as pessoas que se encontravam como "recusas" anteriores aos coordenadores da Coorte de Nascimentos de 2004. 
Nos meses de estágio no Centro de Pesquisa Epidemiológicas da UFPel, participei de palestras e aulas do departamento e fui aluna do "Curso de Extensão em Epidemiologia Básica”, oferecido pelo Programa de PósGraduação em Epidemiologia do Departamento de Medicina Social da UFPel, com carga horária de 51 horas.

A participação efetiva na pesquisa proporcionou uma aproximação em relação ao delineamento e à implementação de um estudo de coorte. Tratouse de uma valiosa contribuição para a execução do presente projeto de doutorado. A experiência na realização do acompanhamento aos 11 anos de idade foi importante para o andamento do doutorado e para minha formação.

A seguir, apresento fotos (Figuras 4 a 10) do período de estágio e das atividades realizadas no Centro de Pesquisa em Saúde "Dr. Amilcar Gigante", da UFPel, reforçando que obtive autorização de todos os envolvidos para a divulgação das imagens. 


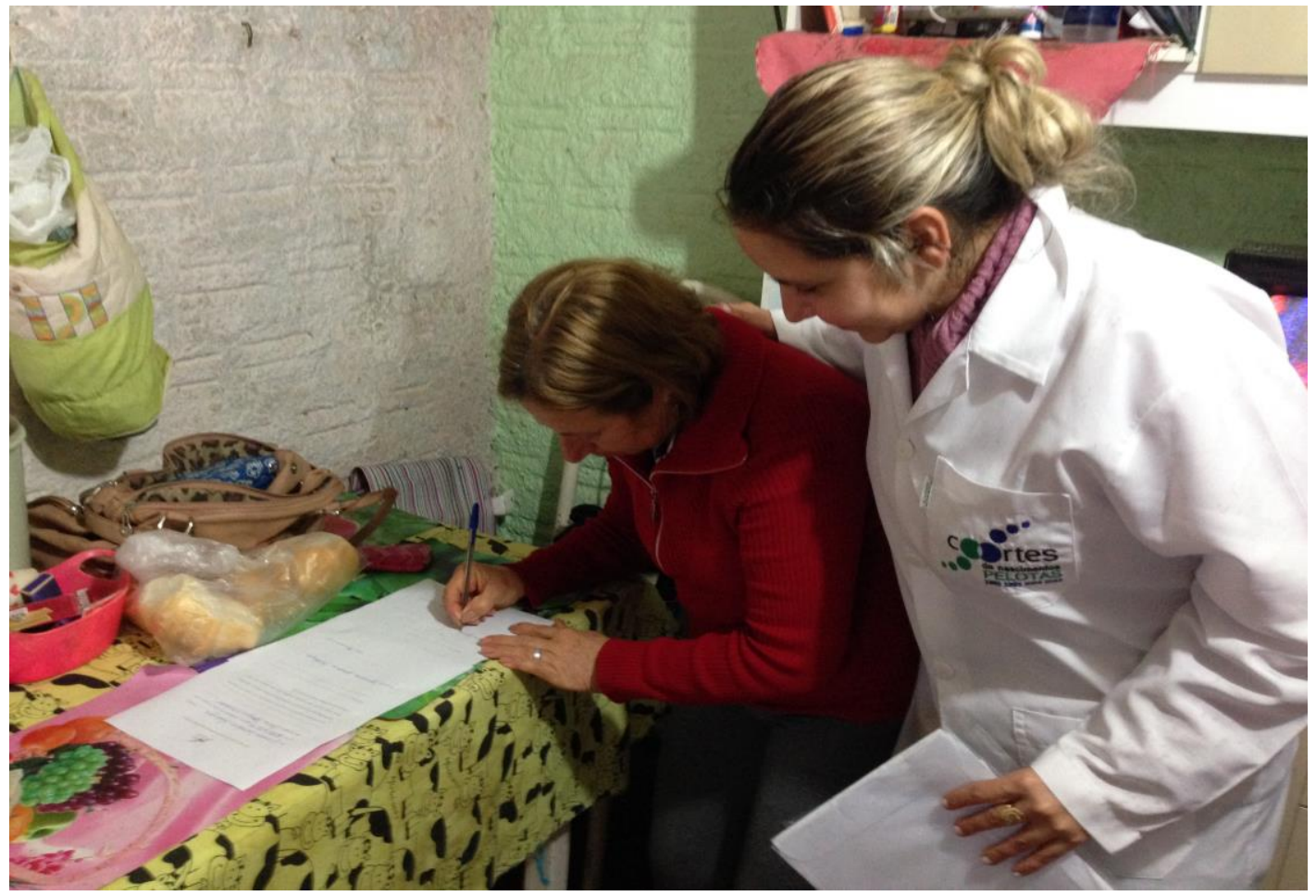

Figura 4 - Assinatura do termo de divulgação de imagem

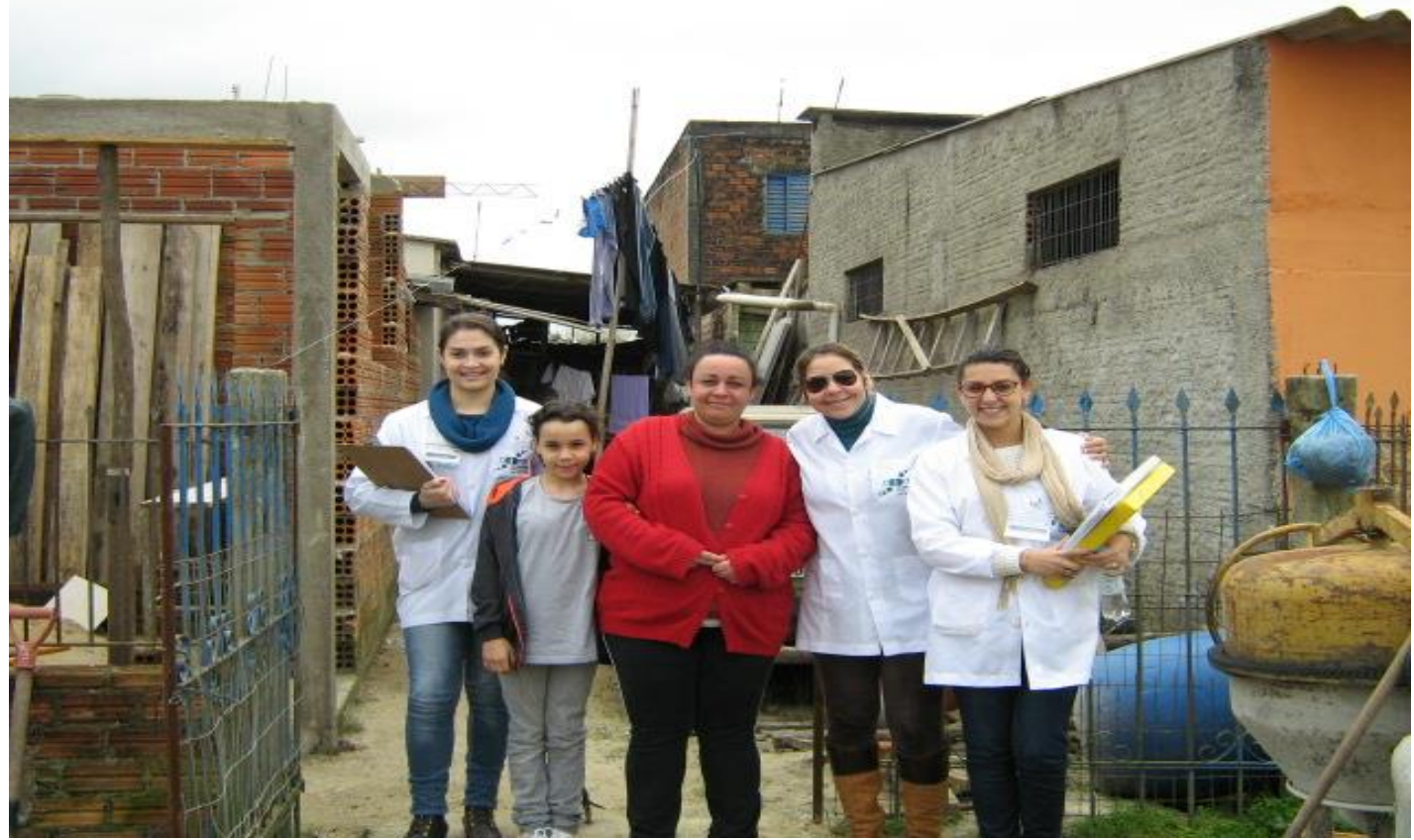

Figura 5 - Equipe de entrevistadoras da coorte de 2004 após entrevista domiciliar 


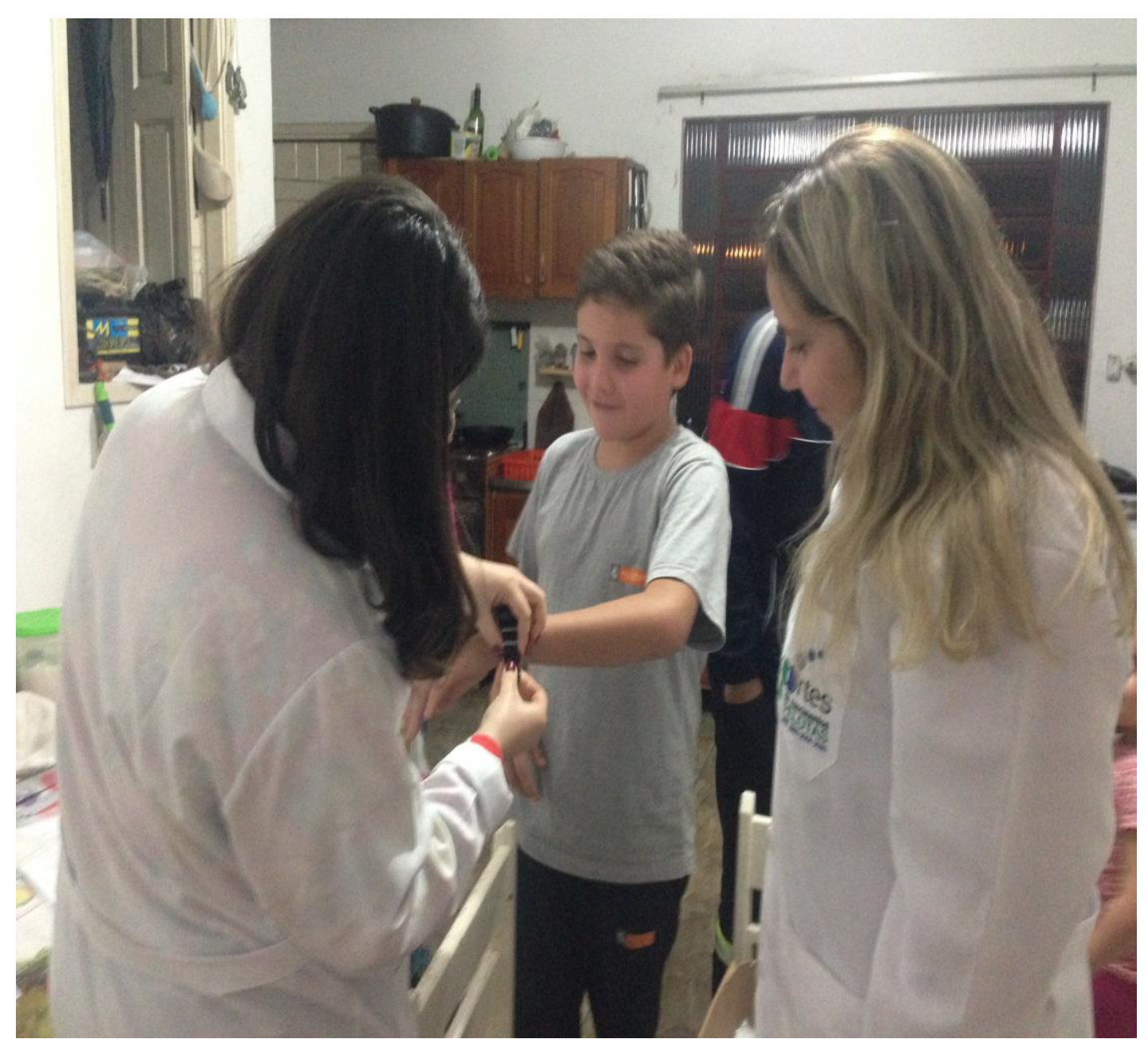

Figura 6 - Colocação do acelerômetro no punho do braço não dominante. Tal aparelho foi utilizado para mensuração objetiva de atividade física, captando os movimentos corporais para estimar os padrões de atividade física dos participantes 


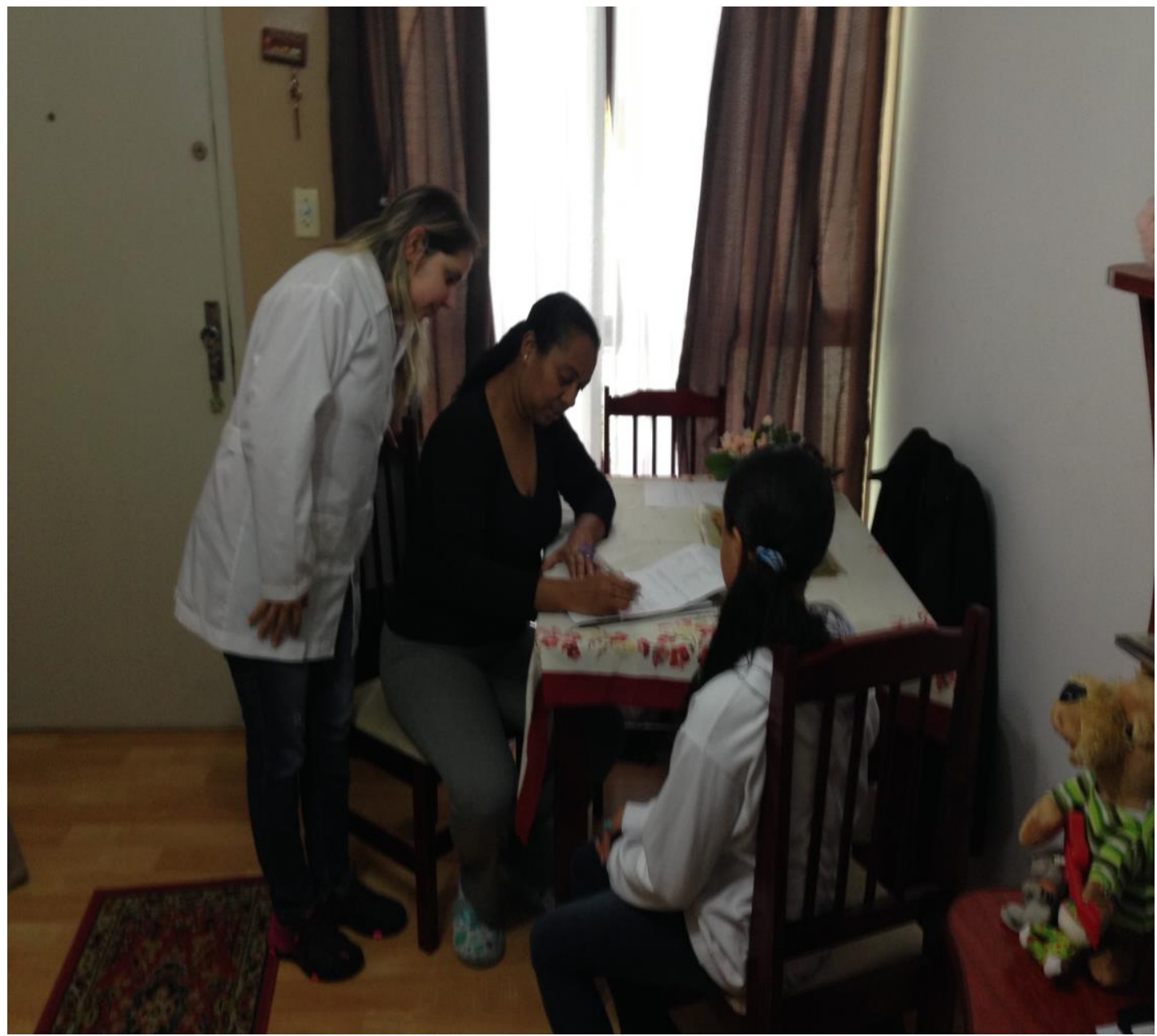

Figura 7 - Assinatura do TCLE 


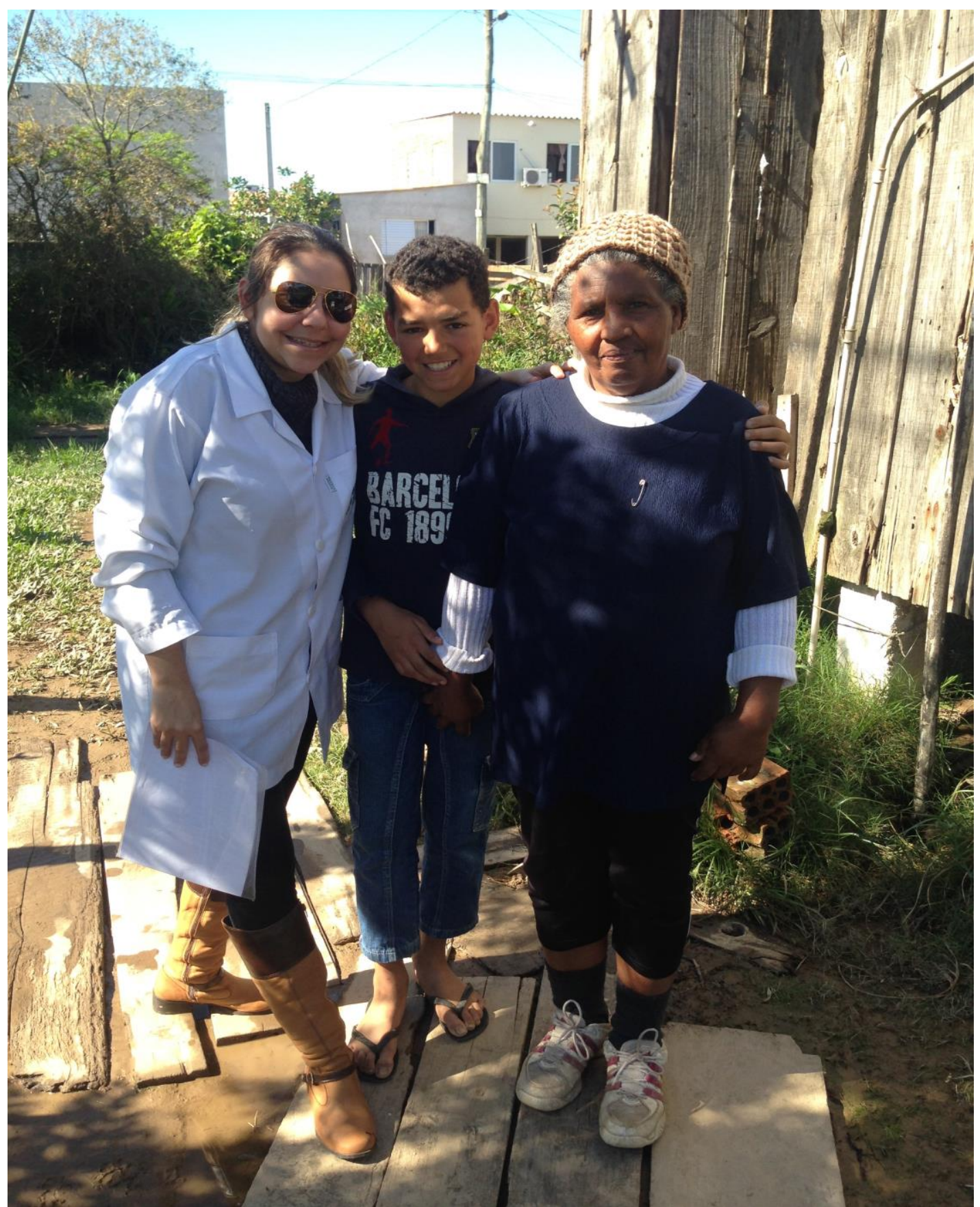

Figura 8 - Família da coorte de 2004 a caminho Centro de Pesquisa em Saúde "Dr. Amilcar Gigante" 


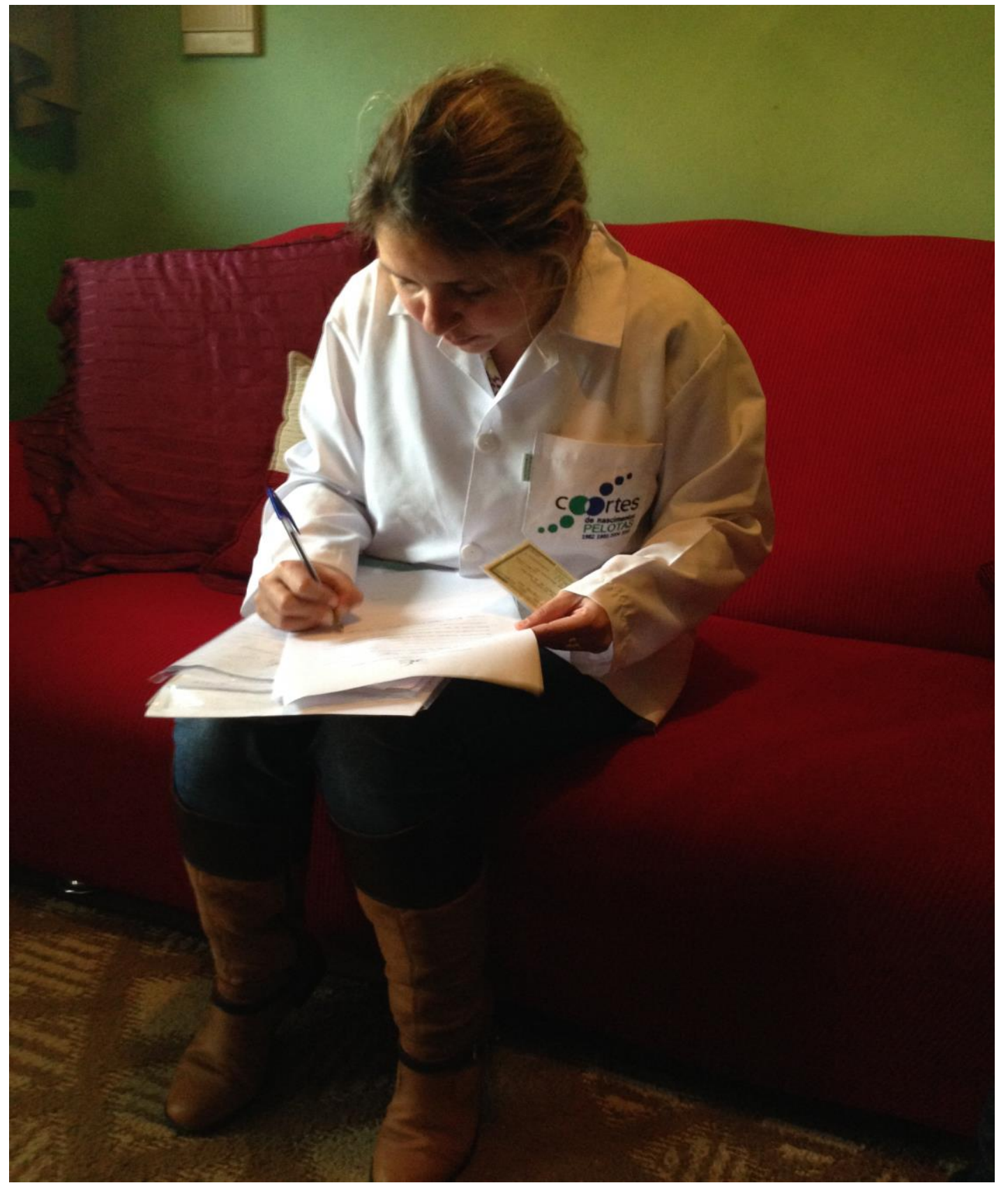

Figura 9 - Preenchimento dos dados pessoais durante entrevista familiar da coorte de 2004 


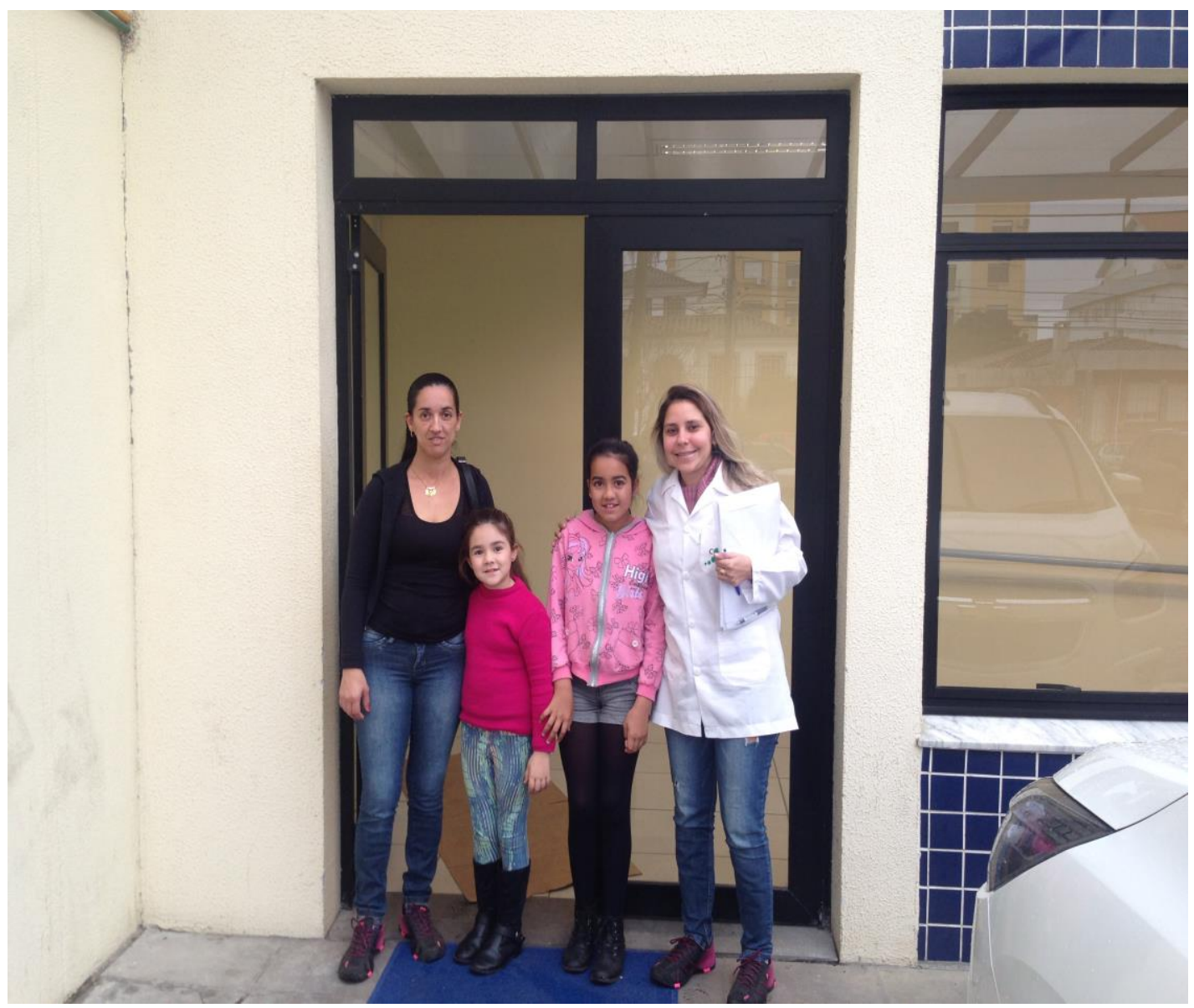

Figura 10 - Família no Centro de Pesquisa em Saúde "Dr. Amilcar Gigante", para realizar os exames de pletismografia por deslocamento de ar (BodPod), densitometria (DXA), avaliação das dimensões corporais (Photonic Scanner), antropometria (altura, altura sentado e circunferência da cintura) e pressão arterial 
"O pensamento parece uma coisa à toa mas como é que a gente voa quando começa a pensar..." Lupcínio Rodrigues 


\section{CONSIDERAÇÕES ÉTICAS}

O projeto foi aprovado pelo Comitê de Pesquisa da Faculdade de Medicina da Universidade de São Paulo em sessão de 18 de março de 2015 (Protocolo de Pesquisa n. 015/15) e pelo Comitê de Ética em Pesquisa da Universidade Federal de Pelotas (UFPel). Foi obtido o termo de consentimento livre e esclarecido das mães ou responsáveis e o termo de assentimento dos adolescentes, assim como foi garantida a confidencialidade dos dados, a participação voluntária e a possibilidade de abandonar o estudo a qualquer momento, sem necessidade de justificativa. Os casos graves de problemas de saúde psiquiátricos identificados pelas psicólogas foram avaliados e, quando necessário, foram encaminhados para os serviços de atendimento psiquiátrico ou psicológico disponíveis na cidade. 
"E tudo ficou tão claro o que era raro, ficou comum como um dia depois do outro como um dia, um dia comum..." Humberto Gessinger 
6. ARTIGO 1 - PREVALÊNCIA E FATORES DE RISCO DOS TRANSTORNOS PSIQUIÁTRICOS NO INÍCIO DA ADOLESCÊNCIA: COORTE DE NASCIMENTOS DE PELOTAS DE 2004

Este capítulo apresenta o artigo "Prevalence and risk factors of psychiatric disorders in early adolescence: 2004 Pelotas (Brazil) birth cohort" de autoria de Carolina La Maison, Tiago N. Munhoz, Iná S. Santos, Luciana Anselmi, Fernando C. Barros, Alicia Matijasevich.

O artigo foi originalmente publicado em acesso aberto (open access) na revista Social Psychiatry and Psychiatric Epidemiology e pode ser acessado gratuitamente no link:

Para citação: La Maison, C., Munhoz, T.N., Santos, I.S. et al. Soc $\begin{array}{llll}\text { Psychiatry } & \text { Psychiatr } & \text { Epidemiol } & \text { (2018) }\end{array}$ https://doi.org/10.1007/s00127-018-1516-z 


\subsection{Abstract}

Purpose: The present study aimed to evaluate the prevalence of psychiatric disorders in early adolescence, to examine the distribution of psychiatric disorders by maternal and child characteristics and to evaluate the occurrence of psychiatric comorbidities. Methods: This was a prospective cohort study of all live births in the city of Pelotas, Brazil, in 2004 ( $n=4,231)$. A total of 3,562 subjects were evaluated at 11 years of age. Psychiatric disorders were assessed using the Development and Well-Being Assessment. Crude and adjusted logistic regression was used to investigate risk factors for any psychiatric disorder. Results: According to DSM-5 criteria, the overall prevalence of psychiatric disorders was 13.2\% ( $n=471)$, $15.6 \%$ among the boys and $10.7 \%$ among the girls. The most common disorders were anxiety disorders (4.3\%), any attention deficit/hyperactivity disorder (4.0\%) and any conduct/oppositional disorder (in 2.8\%). Low maternal education, smoking during pregnancy, the presence of moods symptoms during pregnancy or maternal chronic and severe depressive symptoms in the first years of the adolescent's life, male gender, 5-minute Apgar score $<7$ at birth and preterm birth were associated with higher odds of any psychiatric disorder at age 11. Psychiatric comorbidities were observed in 107 subjects (22.7\%), of whom 73,24 , and 10 had two, three, and four psychiatric diagnoses, respectively. Conclusions: Our results underscore the importance of psychiatric disorders as a prevalent condition in early adolescence, which has a direct impact on the planning of public policies and specific mental health care services in this age group.

Keywords: Mental Disorders; Neurodevelopmental Disorders; Prospective Studies; Adolescent.

\subsection{INTRODUCTION}

Psychiatric disorders account for a significant proportion of the global burden of disease [1,2]. Psychiatric disorders represent $9.8 \%$ of the burden of disease in low- and middle-income countries [3]. According to Kieling et al. 
[1], at least $10 \%$ of children and adolescents worldwide have a psychiatric disorder, a major cause of morbidity and mortality. Among individuals between 10 and 24 years of age, psychiatric disorders account for $45 \%$ of all years lived with disability [4]. In addition, psychiatric disorders and substance use have been found to be the main causes of disability-adjusted life-years (DALYs) among young people in high-income countries []ㅡ.

In adolescents, psychiatric disorders not only cause personal and family suffering but also are recognized risk factors for substance abuse and criminality, as well as predicting negative outcomes in adulthood [6]. A significant proportion of psychiatric disorders diagnosed in adults have their roots in childhood and adolescence [7].

A recent systematic review and meta-analysis of the prevalence of psychiatric disorders in adolescents in 27 countries reported an overall prevalence of psychiatric disorders of $13.4 \%(95 \% \mathrm{Cl}: 11.3-15.9)$. Having duly adjusted for methodological factors, the authors observed a great similarity in the frequency of psychiatric disorders among different countries and cultures [8]. Studies conducted in Brazil have reported the prevalence of psychiatric disorders in adolescence to range from $7 \%$ to $20 \%$, depending on the region investigated, the level of exposure to risk factors, and the methodology used [1].

A wide variety of scales and questionnaires are available for the measurement of psychiatric disorders in low- and middle-income countries $[\underline{9}, \underline{10}]$. Prioritizing either the sensitivity (screening criteria) or specificity (diagnostic criteria) of such tests can result in differences in measures of 
disease occurrence. Higher rates of psychiatric disorders are observed in studies that apply screening criteria. A systematic review and meta-analysis of the prevalence of major depressive disorder, including 116 studies, found that studies using symptom scales reported a higher prevalence of the disorder than did those using established criteria such as those outlined in the DSM-IV and ICD-10 [11].

There is little evidence of specific associations between particular risk factors and particular psychiatric disorders. The most reasonable explanation for the development of psychiatric disorders might be the combination, sequence, and interaction of individual, familial, genetic, and environmental risk factors $[12,13]$. Risk factors for psychiatric disorders range from the biological, genetic, and perinatal to the gender of the child, parental attachment, and trauma, as well as demographic and socioeconomic factors. Among the environmental risk factors associated with psychiatric disorders, the findings related to socioeconomic position are the most robust in epidemiological studies, which have shown an inverse association, a lower socioeconomic position increasing the probability of the individual developing a psychiatric disorder $[\underline{14}, \underline{15}]$.

The frequency and type of psychiatric disorder occurring during adolescence varies according to the gender of the individual. Various studies have suggested that, among adolescents, conduct/oppositional disorders and attention deficit/hyperactivity disorder (ADHD) are more common in males, whereas anxiety and depressive disorders are more common in females [16]. 
Externalizing problems—conduct disorder and oppositional defiant disorder (ODD) - tend to improve with age, whereas internalizing problems - anxiety and depression—tend to worsen [17]. Psychiatric disorders rarely occur in isolation, and comorbidity with other psychiatric disorders is common [18]. The presence of psychiatric comorbidities complicates the diagnostic process, having a significant impact on the natural history, prognosis, and treatment of the primary psychiatric disorder [19]. Two or more comorbid psychiatric disorders reportedly occur in approximately $16 \%$ of adolescents [20].

The present study had the following objectives: to evaluate the prevalence of psychiatric disorders in individuals at the beginning of adolescence (11 years of age); to examine the distribution of psychiatric disorders by gender, socioeconomic position (SEP) and other maternal and child characteristics; and to evaluate the occurrence of psychiatric comorbidities.

\subsection{METHODS}

\subsubsection{Setting and Study Design}

The city of Pelotas is in the south of Brazil, within the state of Rio Grande do Sul. It has a population of approximately 328,000 inhabitants, of which $93.3 \%$ live in the urban area of the city. In 2010 , the city had an adult literacy rate of $95.7 \%$, higher than the $72.0 \%$ reported for Brazil as a whole, and the mean Human Development Index was 0.816, compared with 0.699 for the country as a whole. 
In 2004, all children born at hospitals to mothers living within the urban area of the city of Pelotas were identified and their mothers were invited to participate in a cohort study. The follow-up group comprised 4,231 children born to the mothers who agreed to participate in the study (refusals $<1 \%$ ). A detailed description of the methodology employed can be found elsewhere [21]. Members of the Pelotas 2004 Birth Cohort were evaluated at various time points. The sixth follow-up evaluation of the cohort was conducted between May and October 2015 (follow-up rate of 87\%), when the mean age of the subjects was 11.0 years $(S D=0.3)$.

\subsubsection{Evaluation of Psychiatric Disorders}

The Development and Well-Being Assessment (DAWBA) combines closed and open-ended (qualitative descriptions) questions based on DSMIV, DSM-5 and ICD-10 diagnoses [22].

The following diagnoses were assessed: separation anxiety disorder; specific phobia; social phobia; generalized anxiety disorder; post-traumatic stress disorder; panic disorder and agoraphobia; obsessive-compulsive disorder; depressive disorder, bipolar disorder, and ADHD; ODD; conduct disorder; autism spectrum disorders, eating disorders, and tic disorder, including three new DSM-5 diagnoses-body dysmorphic disorder, disruptive mood dysregulation disorder (DMDD), and binge-eating disorder.

We employed the Portuguese-language version of the DAWBA, which has been cross-culturally adapted and validated for use in Brazil [23] which was administered to mothers or caregivers by trained psychologists. 
The training included a theoretical and a practical phase. The theoretical phase consisted of lessons on the various psychopathologies addressed in the DAWBA, the way the DAWBA instruments work, and the precautions to be taken in their application. The practical phase consisted of supervised interviews with the mothers of children treated at the Pediatric Outpatient Clinic or Psychiatric Clinic of the School of Medicine of the Federal University of Pelotas. The training lasted for 40 hours. During the fieldwork, the psychologists were supervised on a weekly basis, their questions being answered and explanations about the DAWBA being repeated, the supervisory sessions therefore functioning as ongoing reinforcement of the training. The clinical evaluation of the total sample was performed by a psychologist, and a second independent psychologist evaluated $10 \%$ of the study sample. Both were trained in how to apply the DAWBA, in a standardized manner, by the child psychiatrist who had translated and validated the questionnaire for use in Brazil [24]. The interrater agreement was $91.2 \%$ for the presence of any psychiatric disorder, $75.9 \%$ for any anxiety disorder, $73.5 \%$ for any depressive disorder, $72.7 \%$ for ADHD, $72.9 \%$ for conduct disorder, $85.6 \%$ for any autism spectrum disorder, $59.5 \%$ for any eating disorder, and $52.4 \%$ for any tic disorder. We described the DAWBA diagnoses in a dichotomous way (yes/no), strictly adhering to the criteria defined for the diagnostic classifications mentioned above. 


\subsubsection{Covariates}

We evaluated the following demographic and socioeconomic characteristics of the mother at the time of childbirth: age $(\leq 19,20-34$ or $\geq 35$ years); marital status (married/living with a partner or single/divorced/widowed); self-reported skin color (White or Black/mixed); parity (0, 1 or $\geq 2$ previous pregnancies) and socioeconomic position (SEP).

SEP was determined on the basis of the wealth index and the maternal level of education. The wealth index can be used as a general indicator of material living standards. It takes into consideration durable consumer goods (e.g., televisions, cars, computers, and stoves) and household characteristics (e.g., number of toilets), through principal component analysis. For our analyses, the first component was selected and categorized in quintiles [25]. The maternal level of education was categorized as $<5,5-8$, or $\geq 9$ years of formal schooling.

Mood symptoms during pregnancy were identified on the basis of an affirmative response to the question: "During pregnancy, did you feel depressed or have any nervous condition?" Smoking and alcohol use during pregnancy were self-reported and were evaluated retrospectively at birth. Regular smokers were defined as those women who smoked at least one cigarette daily in any trimester of pregnancy. Any amount of alcohol intake during any trimester of pregnancy was considered as alcohol consumption during pregnancy.

The following characteristics of the adolescent were evaluated at birth: gender; multiple pregnancy; 5-minute Apgar score ( $<7$ or $\geq 7$ ); low birthweight 
(LBW, $<2500 \mathrm{~g})$; and preterm birth $(<37$ weeks of gestational age). Assessment of maternal depressive symptoms was made using the Edinburgh Postnatal Depression Scale (EPDS) [26].

The EPDS questionnaire comprises 10 questions that assess symptoms of depression in the last seven days. The EPDS was administered to all of the mothers at each follow-up, except at the 3-month follow-up visit, when it was completed by a subsample of 965 mothers. Trajectories of maternal depressive symptoms in the first years of the adolescents' life were estimated using a semiparametric group-based modeling, a form of finite mixture modeling proposed by Nagin \& Odgers [27]. A censored normal model was adjusted to the data. Five trajectories of maternal depressive symptoms were estimated: low and moderate-low-symptoms, increasing, decreasing and high-chronic maternal depressive symptomatology [28].

\subsubsection{Statistical Analysis}

To describe the characteristics of the sample we used absolute and relative frequencies. To examine the association between psychiatric disorders and gender and SEP, psychiatric disorders were organized in the following groups: any psychiatric disorder; any anxiety disorder; any mood disorder; any ADHD/hyperactivity disorder; any conduct/oppositional disorder; any autism spectrum disorder; any tic disorder/Tourette syndrome and any eating disorder.

The statistical tests were based on the chi-square test. Logistic regression was used to perform bivariate and multivariate analyses, the results of which were expressed as odds ratio (OR) and $95 \% \mathrm{Cl}$. The 
adjusted analysis employed a conceptual hierarchical model to determine the risk factors for any psychiatric disorder. That model consisted of five levels: demographic and socioeconomic variables; variables related to maternal behavioral traits; mood symptoms during pregnancy; characteristics of the child at birth; and trajectories of maternal depressive symptoms between 3 months and 6 years after the birth of the child. The model considers the effect of each variable in relation of the outcome, controlling for confounding among variables of the same or higher level. Variables presenting $p<0.20$ in the adjusted analysis were maintained in each hierarchical level. Statistical analyses were performed using the program Stata, version 13 (StataCorp LP, College Station, TX).

\subsubsection{Ethical Aspects}

The study was approved by the Research Committee of the University of São Paulo School of Medicine, (Research Protocol no. 015/15), and by the Research Ethics Committee of the Federal University of Pelotas. Written informed consent was obtained from the mothers or legal guardians of the adolescents. At the 11-year follow-up, adolescents also signed an informed consent form. Cases of severe mental health problems, as identified by the psychologists, were evaluated and, when necessary, were referred to the psychiatric or psychological care facilities available in the city.

\subsection{RESULTS}

6.4.1 Attrition analysis and characteristics of the sample 
Of the 4231 participants constituting the original cohort, 98 died in the first eleven years of life and 3566 were interviewed at eleven years. Data about psychiatric disorders at 11 years were available on 3562 adolescents (84.2\% of the original cohort). Mothers of participants who remained in the study were more wealthy, educated and less likely to be single, multiparous, smokers and to report mood symptoms during pregnancy (Table 1). The final sample also included a smaller proportion of subjects who had been low birthweight or preterm neonates, as well as fewer subjects who had a 5minute Apgar score $<7$ at birth compared to those not included in the present analyses.

Amongst included participants, $44 \%$ of the mothers had studied 9 or more years and nearly $70 \%$ of them were between 20 and 34 years old when the adolescent was born. For $40 \%$ of the mothers, the cohort participant was the first child, and $16 \%$ of them were not living with a partner at the time of delivery. Almost $27 \%$ of the mothers smoked during pregnancy, $3 \%$ consumed any alcohol beverage and one-fourth of them reported mood symptoms during pregnancy. At birth, approximately $52 \%$ of the subjects were male, $2 \%$ were twins, $10 \%$ were LBW neonates, $14 \%$ premature and less than $2 \%$ presented a 5 -minute Apgar score $<7$.

\subsubsection{Prevalence of Psychiatric Disorders}

We found that $13.2 \%$ and $11.3 \%$ of the adolescents, respectively, met at least one DSM-5 or ICD-10 diagnostic criterion for a psychiatric disorder (Table 2).

The most common psychiatric diagnoses were anxiety disorders, which were identified in approximately $4 \%$ of the adolescents. The most common anxiety disorders were specific phobia and separation anxiety. The prevalence of depressive disorders was $0.8 \%$ according to the DSM-IV criteria, 3.0\% according to the DSM-5 criteria (which include DMDD), and 
$1.2 \%$ according to the ICD-10 criteria. The prevalence of DMDD and body dysmorphic disorder, categories present only in the DSM-5, was $2.4 \%$ and $0.2 \%$, respectively. The overall prevalence of mood disorders was $3.2 \%$ when the DSM-5 criteria were applied and 1.2\% when the ICD-10 criteria were applied. According to the DSM-5 criteria, the prevalence of ADHD or other hyperactivity disorder was $4.0 \%$, the combined type of ADHD being predominant (occurring in 2.1\%). Using the ICD-10 diagnostic classification, we found that the overall prevalence of ADHD or other hyperkinetic disorder was $3.4 \%$, that of hyperkinesia being $2.1 \%$ and that of ADHD not otherwise specified being $1.3 \%$. The prevalence of conduct disorder and ODD was $2.8 \%$ and $3.3 \%$ according to the DSM-5 and ICD-10, respectively. Autistic spectrum disorders and eating disorders were rarely found among the adolescents.

\subsubsection{Risk factors for psychiatric disorders}

The proportion of adolescents with at least one psychiatric disorder was greater among the boys than among the girls (Table 3 ). The prevalence of any ADHD/ hyperactivity disorder, any conduct/oppositional disorder, and any tic disorder/Tourette syndrome was nearly twice as high among male subjects as among female subjects. Although low in both genders, the prevalence of eating disorders was higher among the girls than among the boys.

The prevalence of any psychiatric disorder, any depressive disorder, any $\mathrm{ADHD}$ / hyperactivity disorder, and any conduct/oppositional disorder was lower among adolescents belonging to wealthier families than among those 
belonging to poorer families (Table 4). The prevalence of any psychiatric disorder, any depressive disorder, any ADHD/hyperactivity disorder, and any conduct/oppositional disorder was lower among adolescents whose mothers had $\geq 9$ years of schooling than among those whose mothers had $<9$ years of schooling.

In the adjusted analysis, the odds of developing any psychiatric disorder by age 11 was higher among adolescents of less educated women, smokers and those with depressive symptoms during pregnancy (Table 5). Adolescents of mothers in the "high-chronic" depressive trajectory group were more than four-times as likely to have any psychiatric disorder compared to adolescents of women in the "low" group. Male gender, 5minute Apgar score $<7$ at birth and preterm birth were associated with higher risk of any psychiatric disorder at age 11.

\subsubsection{Psychiatric comorbidity}

Among adolescents with psychiatric disorders $(n=471)$, two psychiatric disorders were observed in $73(15.5 \%)$, three were observed in $24(5.1 \%)$ and four psychiatric disorders were observed in 10 (2.1\%).

The various diagnostic combinations are detailed in the Electronic Supplementary Material (Table 1). Among the 73 adolescents diagnosed with two psychiatric disorders, the most common combination was any mood disorder with any ADHD/hyperactivity disorder, which was seen in 18 (24.6\%), followed by any anxiety disorder with any mood disorder, seen in 16 (21.9\%), any ADHD/hyperactivity disorder with any conduct/oppositional 
disorder, seen in $13(17.8 \%)$, and any anxiety disorder with any ADHD/ hyperactivity disorder, seen in $10(13.7 \%)$.

\subsection{DISCUSSION}

The prevalence of a diagnosis of any psychiatric disorder at age 11 was $13.2 \%$ and $11.3 \%$, respectively, when the DSM-5 and ICD-10 criteria were applied. The prevalence of any psychiatric disorder was higher among the male adolescents, mainly because ADHD/hyperactivity disorders and conduct/oppositional disorders were more common among the boys.

The prevalence of any mood disorder, any ADHD/ hyperactivity disorder, and any psychiatric disorder was higher among adolescents belonging to families with a lower SEP than among those belonging to families with a higher SEP.

Low maternal education, smoking during pregnancy, the presence of moods symptoms during pregnancy or maternal chronic and severe depressive symptoms in the first years of the adolescent's life, male gender, 5-minute Apgar score $<7$ at birth and preterm birth were associated with higher odds of any psychiatric disorder at age 11.

The prevalence of psychiatric disorders among the adolescents in our sample is comparable to that reported in a recent systematic review and meta-analysis that included studies conducted in 27 countries and employed various methods of assessing psychiatric disorders [8]. Other studies from low- and middle-income countries, in which the DAWBA was applied, reported prevalence rates similar to those of our study. In a two-stage prevalence study of randomized samples of children between 5 and 10 years 
of age $(\mathrm{N}=922)$ from three contrasting areas of Bangladesh [24], the overall prevalence of any psychiatric disorder was $15.2 \%$ (15.4\% in rural areas, $10.0 \%$ in urban areas with a better SEP, and $19.5 \%$ in urban areas with a worse SEP). In a sample of 448 subjects between 7 and 14 years of age in Russia [29], the observed prevalence of psychiatric disorders was $15.3 \%$, $70 \%$ higher than that previously observed in a comparable study of children and adolescents in Great Britain [30]. In a study involving subjects between 7 and 14 years of age in the southeastern region of Brazil [23], the reported prevalence of psychiatric disorders was $12.7 \%$.

The rates of psychiatric disorders are generally higher in studies using screening instruments than in those using diagnostic instruments. A study conducted in the city of Taubaté, in the state of São Paulo, Brazil, evaluated 454 schoolchildren (7-11 years of age) at public and private schools [31]. Using the Strengths and Difficulties Questionnaire, the authors found that $35.2 \%$ of the subjects were considered positive for mental health problems, which reached clinical relevance in $22.7 \%$ and borderline relevance in $12.5 \%$.

The Estudo de Riscos Cardiovasculares em Adolescentes (ERICA, Study of Cardiovascular Risk in Adolescents), a cross-sectional school-based study conducted in Brazilian municipalities with more than 100,000 inhabitants, evaluated 74,589 adolescents [32]. Using the 12-item General Health Questionnaire, the authors found that, among the subjects between 12 and 14 years of age, the prevalence of common psychiatric disorders was $26.7 \%$. 
In the present study, the evaluation of the prevalence and symptomatology of psychiatric disorders was performed according to the DSM-5 criteria. Therefore, in addition to the specific differences between the DSM-IV and DSM-5, we included three nosological categories belonging exclusively to the DSM-5: DMDD; body dysmorphic disorder; and bingeeating disorder. DMDD, which is included in the DSM-5 depressive disorders section, was the main factor accounting for the difference of mood disorders prevalence between the DSM-5 and ICD-10 criteria. It should be noted that, according to the DSM-5, the symptoms of ODD often occur in children with DMDD, the main difference being that angry outbursts and the occurrence of the symptoms in more than one sphere (family, school, and social) are more common in the latter. Therefore, this new diagnostic category (DMDD) also explains the difference between prevalence rates for conduct/oppositional disorders when ICD-10 and DSM-5 are used.

In our study, the most common psychiatric disorders were anxiety disorders. A systematic review of 11 studies, using the DSM-III, Revised or DSM-IV diagnostic criteria, showed that the reported prevalence of any anxiety disorder in children under 12 years of age ranged from $2.6 \%$ to $41.2 \%$ [33].

The authors found that the most common anxiety diagnosis in that age group was separation anxiety disorder. Despite the notable variation in prevalence estimates, which is likely due to differences in methodology and the instruments used, the lifetime prevalence of "any anxiety disorder" in studies involving children or adolescents is between $15 \%$ and $20 \%$ [34]. 
In the present study, the prevalence of any ADHD/ hyperactivity disorder was similar to estimates reported for children and adolescents around the world (5.3\% in individuals under 18 years of age) [19]. A recent cross-sectional study that assessed 1,676 6-to-16 year-olds from four regions of Brazil using the Schedule for Affective Disorders and Schizophrenia for School-Age Children/Present-and-Lifetime-Version (K-SADS-PL) applied to mothers/main caregivers, reported a prevalence of any ADHD/ hyperactivity disorder of $4.5 \%(\mathrm{Cl} 95 \% 3.5-5.6)$ [35].

Epidemiological and clinical studies have found gender-related differences in many types of psychopathology. Early-onset disorders (those that begin in childhood) are typically more prevalent in boys, whereas those that begin in adolescence are more prevalent in girls [36].

Such differences vary by age group. For example, most studies involving children report that the prevalence of conduct disorders, with aggressive and antisocial behaviors, is higher among boys than among girls. During adolescence, the prevalence of depression and eating disorders is higher among girls, who are also more likely to engage in suicidal ideation. Adolescent boys, however, have more problems with anger, more often engage in high-risk behaviors, and are more likely to commit suicide [37].

Contrary to what was observed in the ERICA study, in which the prevalence of common psychiatric disorders in subjects 12-14 years of age was higher in girls than in boys, in our study the prevalence of any psychiatric disorder was higher in the boys than in the girls [32]. Because our study involved subjects age 11 years, the frequency of disorders by gender found 
in the current study may reflect the prevalence of disorders observed in childhood, which is different from the prevalence of disorders by gender observed in older adolescents.

The SEP is used in epidemiological studies as a measure of socioeconomic factors that influence the place an individual occupies within the structure of society [38]. Although there are several indicators of the SEP, schooling, income, and the wealth index are the most widely used. Poverty is associated with multiple environmental risk factors for psychopathology, such as parental unemployment, maternal anxiety/depression, child/adolescent maltreatment and trauma exposure and fewer resources to access good quality health care.

A systematic review that evaluated the effects of the SEP on the occurrence of psychiatric disorders in children and adolescents reported that the prevalence of psychiatric disorders was higher among children and adolescents belonging to families with a low income and whose parents had a low level of education than among those whose parents had higher incomes or higher levels of education [39]. In our study, after adjustment for potential confounders, only poor maternal schooling was associated with higher risk of any psychiatric disorder at age 11.

In accordance with our results, many studies reported the association between fetal exposure to maternal smoking in pregnancy and several adverse offspring mental health outcomes $[\underline{40}, \underline{41}]$. 
Recently, Talati et al. showed that birthweight did not mediate the association between the exposure and externalizing psychopathology, indicating that the mechanisms through which maternal smoking increases the risk of offspring psychopathology were not operating through lower birthweight [42].

Consistent with previous reports, we found that maternal depression, especially when it is chronic and severe, was a strong predictor of offspring psychiatric disorders $[\underline{43}, \underline{44}]$. Even though the exposure to maternal depression appears to be a significant independent risk factor for offspring mental disorders, Barker et al. reported that multiple risk factors exposures strongly affect child psychopathology, increasing the risk over and above the influence of maternal depression [45].

In our study, both preterm birth and 5-minute Apgar score $<7$ were associated with psychiatric disorders at age 11. The Apgar score is widely used to report the status of the newborn infant immediately after birth. Even though numerous factors can influence the Apgar score, 5-minute low Apgar scores have been associated with an increased risk of a wide range of neurological and psychiatric disorders $[\underline{46}, \underline{47}]$. Preterm birth has also been identified as a risk factor for several psychiatric disorders in childhood, such as emotional problems, ADHD and autism spectrum, increasing the risk significantly as gestational age decreases $[\underline{48}, \underline{49}]$.

In the present study, $22.7 \%$ of the adolescents with psychiatric disorders had one or more psychiatric comorbidities. Anxiety disorders accompany most psychiatric disorders, including mood disorders, disruptive 
behaviors, eating disorders, and substance use disorders. The combination of anxiety disorders and mood disorders is so common that many authors have postulated that anxiety disorders are part of the developmental sequence in which anxiety is expressed early in life, followed by depression in adulthood [34].

Community studies in young people have shown a high degree of the co-occurrence of conduct disorders and ADHD, which is associated with a worse prognosis in behavioral disorders, including substance use disorders [50]. Disruptive behavior disorders are also frequently accompanied by mood and anxiety disorders, although parents and teachers often report fewer problems related to anxiety and mood than to externalizing disorders, which are more easily perceived and more difficult to manage.

The presence of psychiatric comorbidity interferes with the evolution of the psychiatric disorder, making its course more chronic, with a worse prognosis and a worse response to treatment [18]. The prevalence of comorbidities tends to be higher in studies conducted at mental health facilities and such comorbidities tend to occur more frequently in the later stages of development. A recent study by Bordin et al. showed that comorbidity increased the likelihood of maternal recognition of emotional/behavioral problems in children and adolescents, an essential first step in the searching for treatment or support for their children [51].

\subsubsection{Strengths and Limitations of the Study}

Among the advantages of the study is that it was a population-based study, with face-to-face interviews, involving a large sample of adolescents. 
In addition, we used an internationally recognized instrument, designed to generate diagnoses of psychiatric disorders, that has been validated for use in Brazil and was applied by trained psychologists, thus ensuring the quality of the data. Another important point is that we employed the criteria of the recently published DSM-5, which follows internationally accepted diagnostic criteria. Furthermore, the proportion of non-respondents was low. One limitation of our study is that it was based only on information obtained from the mothers or legal guardians, because the DAWBA was not administered to the adolescents themselves or to their teachers. Reports from teachers and self-reports from adolescents could reveal other symptoms not recognized by the mothers, contributing to a more accurate diagnosis of the psychiatric disorders.

\subsection{Conclusions}

Our findings underscore the relevance of psychiatric disorders in early adolescence and provide evidence for the development of strategies for the prevention of psychiatric disorders, as well as for the promotion and recovery of mental health, during childhood and adolescence. We also observed a higher frequency of psychiatric disorders in boys and in the adolescents of mothers with chronic depression and with a less favorable SEP. Our results might contribute to reducing the knowledge gap related to the lack of population-based studies on psychiatric disorders in adolescents in low-and middle-income countries, as well as facilitating the planning of specific services to address psychiatric disorders in this age group, minimizing the short- and long-term impact of such disorders along the life-course. 


\subsection{REFERENCES}

1. Kieling C, Baker-Henningham H, Belfer M, Conti G, Ertem I, Omigbodun O, Rohde LA, Srinath S, Ulkuer N, Rahman A (2011) Child and adolescent mental health worldwide: evidence for action. Lancet 378 (9801):1515-1525. doi:10.1016/S0140-6736(11)60827-1

2. Whiteford HA, Degenhardt L, Rehm J, Baxter AJ, Ferrari AJ, Erskine HE, Charlson FJ, Norman RE, Flaxman AD, Johns N, Burstein R, Murray CJ, Vos T (2013) Global burden of disease attributable to mental and substance use disorders: findings from the Global Burden of Disease Study 2010. Lancet 382 (9904):1575-1586. doi:10.1016/S0140-6736(13)61611-6

3. Patel V (2007) Mental health in low- and middle-income countries. Br Med Bull 81-82:81-96. doi:10.1093/bmb/ldm010

4. Gore FM, Bloem PJ, Patton GC, Ferguson J, Joseph V, Coffey C, Sawyer SM, Mathers CD (2011) Global burden of disease in young people aged 1024 years: a systematic analysis. Lancet 377 (9783):2093-2102. doi:10.1016/S0140-6736(11)60512-6

5. Erskine HE, Moffitt TE, Copeland WE, Costello EJ, Ferrari AJ, Patton G, Degenhardt L, Vos T, Whiteford HA, Scott JG (2015) A heavy burden on young minds: the global burden of mental and substance use disorders in children and youth. Psychol Med 45 (7):1551-1563. doi:10.1017/S0033291714002888 
6. de Girolamo G, Dagani J, Purcell R, Cocchi A, McGorry PD (2012) Age of onset of mental disorders and use of mental health services: needs, opportunities and obstacles. Epidemiol Psychiatr Sci 21 (1):47-57

7. Kessler RC, Amminger GP, Aguilar-Gaxiola S, Alonso J, Lee S, Ustün TB (2007) Age of onset of mental disorders: a review of recent literature. Curr Opin Psychiatry 20 (4):359-364. doi:10.1097/YCO.0b013e32816ebc8c

8. Polanczyk GV, Salum GA, Sugaya LS, Caye A, Rohde LA (2015) Annual research review: A meta-analysis of the worldwide prevalence of mental disorders in children and adolescents. J Child Psychol Psychiatry 56 (3):345365. doi:10.1111/jcpp.12381

9. Ali GC, Ryan G, De Silva MJ (2016) Validated Screening Tools for Common Mental Disorders in Low and Middle Income Countries: A

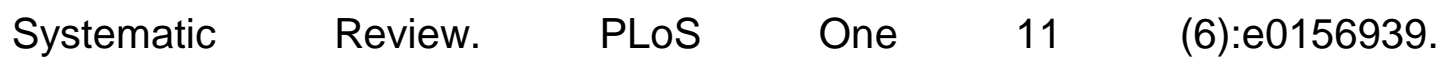
doi:10.1371/journal.pone.0156939

10. Beidas RS, Stewart RE, Walsh L, Lucas S, Downey MM, Jackson K, Fernandez T, Mandell DS (2015) Free, brief, and validated: Standardized instruments for low-resource mental health settings. Cogn Behav Pract 22 (1):5-19. doi:10.1016/j.cbpra.2014.02.002

11. Ferrari AJ, Charlson FJ, Norman RE, Patten SB, Freedman G, Murray CJ, Vos T, Whiteford HA (2013) Burden of depressive disorders by country, sex, age, and year: findings from the global burden of disease study 2010 . PLoS Med 10 (11):e1001547. doi:10.1371/journal.pmed.1001547

12. Ford T, Goodman R, Meltzer H (2004) The relative importance of child, family, school and neighbourhood correlates of childhood psychiatric 
disorder. Soc Psychiatry Psychiatr Epidemiol 39 (6):487-496. doi:10.1007/s00127-004-0782-0

13. Goodman R, Scott S (2012) Child and Adolescent Psychiatry. 3rd edn. Wiley-Blackwell, Oxford

14. Costello EJ, Angold A, Burns BJ, Stangl DK, Tweed DL, Erkanli A, Worthman CM (1996) The Great Smoky Mountains Study of Youth. Goals, design, methods, and the prevalence of DSM-III-R disorders. Arch Gen Psychiatry 53 (12):1129-1136

15. Rutter M (2003) Poverty and child mental health: natural experiments and social causation. JAMA 290 (15):2063-2064. doi:10.1001/jama.290.15.2063

16. Costello EJ, Copeland W, Angold A (2011) Trends in psychopathology across the adolescent years: what changes when children become adolescents, and when adolescents become adults? J Child Psychol Psychiatry 52 (10):1015-1025. doi:10.1111/j.1469-7610.2011.02446.x

17. Salum GA, Polanczyk GV, Miguel EC, Rohde LA (2010) Effects of childhood development on late-life mental disorders. Curr Opin Psychiatry 23 (6):498-503. doi:10.1097/YCO.0b013e32833ead33

18. Merikangas KR, He JP, Burstein M, Swanson SA, Avenevoli S, Cui L, Benjet C, Georgiades K, Swendsen J (2010) Lifetime prevalence of mental disorders in U.S. adolescents: results from the National Comorbidity Survey Replication--Adolescent Supplement (NCS-A). J Am Acad Child Adolesc Psychiatry 49 (10):980-989. doi:10.1016/j.jaac.2010.05.017 
19. Polanczyk G, de Lima MS, Horta BL, Biederman J, Rohde LA (2007) The worldwide prevalence of ADHD: a systematic review and metaregression analysis. Am J Psychiatry 164 (6):942-948. doi:10.1176/ajp.2007.164.6.942 20. Kessler RC, Adler LA, Berglund P, Green JG, McLaughlin KA, Fayyad J, Russo LJ, Sampson NA, Shahly V, Zaslavsky AM (2014) The effects of temporally secondary co-morbid mental disorders on the associations of DSM-IV ADHD with adverse outcomes in the US National Comorbidity Survey Replication Adolescent Supplement (NCS-A). Psychol Med 44 (8):1779-1792. doi:10.1017/S0033291713002419

21. Santos IS, Barros AJ, Matijasevich A, Domingues MR, Barros FC, Victora CG (2011) Cohort profile: the 2004 Pelotas (Brazil) birth cohort study. Int J Epidemiol 40 (6):1461-1468. doi:10.1093/ije/dyq130

22. Goodman R, Ford T, Richards H, Gatward R, Meltzer H (2000) The Development and Well-Being Assessment: description and initial validation of an integrated assessment of child and adolescent psychopathology. J Child Psychol Psychiatry 41 (5):645-655

23. Fleitlich-Bilyk B, Goodman R (2004) Prevalence of child and adolescent psychiatric disorders in southeast Brazil. J Am Acad Child Adolesc Psychiatry 43 (6):727-734. doi:10.1097/01.chi.0000120021.14101.ca

24. Mullick MS, Goodman R (2005) The prevalence of psychiatric disorders among 5-10 year olds in rural, urban and slum areas in Bangladesh: an exploratory study. Soc Psychiatry Psychiatr Epidemiol 40 (8):663-671. doi:10.1007/s00127-005-0939-5 
25. Barros AJ, Victora CG (2005) [A nationwide wealth score based on the 2000 Brazilian demographic census]. Rev Saude Publica 39 (4):523-529. doi:/S0034-89102005000400002

26. Santos IS, Matijasevich A, Tavares BF, Barros AJ, Botelho IP, Lapolli C, Magalhaes PV, Barbosa AP, Barros FC (2007) Validation of the Edinburgh Postnatal Depression Scale (EPDS) in a sample of mothers from the 2004 Pelotas Birth Cohort Study. Cadernos de saude publica 23 (11):2577-2588 27. Nagin DS, Odgers CL (2010) Group-based trajectory modeling in clinical research. Annual review of clinical psychology 6:109-138. doi:10.1146/annurev.clinpsy.121208.131413

28. Matijasevich A, Murray J, Cooper PJ, Anselmi L, Barros AJ, Barros FC, Santos IS (2015) Trajectories of maternal depression and offspring psychopathology at 6 years: 2004 Pelotas cohort study. Journal of affective disorders 174:424-431. doi:10.1016/j.jad.2014.12.012

29. Goodman R, Slobodskaya H, Knyazev G (2005) Russian child mental health--a cross-sectional study of prevalence and risk factors. Eur Child Adolesc Psychiatry 14 (1):28-33. doi:10.1007/s00787-005-0420-8

30. Ford T, Goodman R, Meltzer H (2003) The British Child and Adolescent Mental Health Survey 1999: the prevalence of DSM-IV disorders. J Am Acad Child Adolesc Psychiatry 42 (10):1203-1211. doi:10.1097/00004583200310000-00011

31. Vitolo YL, Fleitlich-Bilyk B, Goodman R, Bordin IA (2005) [Parental beliefs and child-rearing attitudes and mental health problems among 
schoolchildren]. Rev Saude Publica 39 (5):716-724. doi:/S003489102005000500004

32. Lopes CS, Abreu GeA, dos Santos DF, Menezes PR, de Carvalho KM, Cunha CeF, de Vasconcellos MT, Bloch KV, Szklo M (2016) ERICA: prevalence of common mental disorders in Brazilian adolescents. Rev Saude Publica 50 Suppl 1:14s. doi:10.1590/S01518-8787.2016050006690

33. Cartwright-Hatton S, McNicol K, Doubleday E (2006) Anxiety in a neglected population: prevalence of anxiety disorders in pre-adolescent children. Clin Psychol Rev 26 (7):817-833. doi:10.1016/j.cpr.2005.12.002

34. Beesdo K, Knappe S, Pine DS (2009) Anxiety and anxiety disorders in children and adolescents: developmental issues and implications for DSM-V. Psychiatr Clin North Am 32 (3):483-524. doi:10.1016/j.psc.2009.06.002 35. Paula CS, Coutinho ES, Mari JJ, Rohde LA, Miguel EC, Bordin IA (2015) Prevalence of psychiatric disorders among children and adolescents from four Brazilian regions. Rev Bras Psiquiatr 37 (2):178-179. doi:10.1590/15164446-2014-1606

36. Rutter M, Caspi A, Moffitt TE (2003) Using sex differences in psychopathology to study causal mechanisms: unifying issues and research strategies. J Child Psychol Psychiatry 44 (8):1092-1115

37. World Health Organization (2002) Gender and mental health. Geneva 38. Lynch JW, Smith GD, Kaplan GA, House JS (2000) Income inequality and mortality: importance to health of individual income, psychosocial environment, or material conditions. BMJ 320 (7243):1200-1204 
39. Reiss F (2013) Socioeconomic inequalities and mental health problems in children and adolescents: a systematic review. Soc Sci Med 90:24-31. doi:10.1016/j.socscimed.2013.04.026

40. Holz NE, Boecker R, Baumeister S, Hohm E, Zohsel K, Buchmann AF, Blomeyer D, Jennen-Steinmetz C, Hohmann S, Wolf I, Plichta MM, MeyerLindenberg A, Banaschewski T, Brandeis D, Laucht M (2014) Effect of prenatal exposure to tobacco smoke on inhibitory control: neuroimaging results from a 25-year prospective study. JAMA psychiatry 71 (7):786-796. doi:10.1001/jamapsychiatry.2014.343

41. Ruisch IH, Dietrich A, Glennon JC, Buitelaar JK, Hoekstra PJ (2017) Maternal substance use during pregnancy and offspring conduct problems: A meta-analysis. Neuroscience and biobehavioral reviews. doi:10.1016/j.neubiorev.2017.08.014

42. Talati A, Wickramaratne PJ, Wesselhoeft R, Weissman MM (2017) Prenatal tobacco exposure, birthweight, and offspring psychopathology. Psychiatry research 252:346-352. doi:10.1016/j.psychres.2017.03.016

43. Campbell SB, Matestic P, von Stauffenberg C, Mohan R, Kirchner T (2007) Trajectories of maternal depressive symptoms, maternal sensitivity, and children's functioning at school entry. Developmental psychology 43 (5):1202-1215. doi:10.1037/0012-1649.43.5.1202

44. Cents RA, Diamantopoulou S, Hudziak JJ, Jaddoe VW, Hofman A, Verhulst FC, Lambregtse-van den Berg MP, Tiemeier H (2013) Trajectories of maternal depressive symptoms predict child problem behaviour: the 
Generation R study. Psychol Med $43 \quad$ (1):13-25. doi:10.1017/S0033291712000657

45. Barker ED, Copeland W, Maughan B, Jaffee SR, Uher R (2012) Relative impact of maternal depression and associated risk factors on offspring psychopathology. The British journal of psychiatry : the journal of mental science 200 (2):124-129. doi:10.1192/bjp.bp.111.092346

46. Grizenko N, Eberle ML, Fortier ME, Cote-Corriveau G, Jolicoeur C, Joober R (2016) Apgar Scores Are Associated with AttentionDeficit/Hyperactivity Disorder Symptom Severity. Canadian journal of psychiatry Revue canadienne de psychiatrie 61 (5):283-290. doi:10.1177/0706743716635544

47. Odd DE, Rasmussen F, Gunnell D, Lewis G, Whitelaw A (2008) A cohort study of low Apgar scores and cognitive outcomes. Archives of disease in $\begin{array}{lllll}\text { childhood Fetal and neonatal edition } 93 & \text { (2):F115-120. }\end{array}$ doi:10.1136/adc.2007.123745

48. Johnson S, Marlow N (2011) Preterm birth and childhood psychiatric disorders. Pediatric research $69 \quad(5 \quad \mathrm{Pt} \quad$ 2):11R-18R. doi:10.1203/PDR.0b013e318212faa0

49. Indredavik MS, Vik T, Evensen KA, Skranes J, Taraldsen G, Brubakk AM (2010) Perinatal risk and psychiatric outcome in adolescents born preterm with very low birth weight or term small for gestational age. Journal of developmental and behavioral pediatrics : JDBP 31 (4):286-294. doi:10.1097/DBP.0b013e3181d7b1d3 
50. Loeber R, Burke JD, Lahey BB, Winters A, Zera M (2000) Oppositional defiant and conduct disorder: a review of the past 10 years, part I. J Am Acad Child Adolesc Psychiatry 39 (12):1468-1484. doi:10.1097/00004583200012000-00007

51. Bordin IA, Curto BM, Murray J (2017) Maternal recognition of child mental health problems in two Brazilian cities. Rev Bras Psiquiatr:0. doi:10.1590/1516-4446-2016-1957 
TABLE 1. Comparison of maternal and child characteristics between those included and not included in the present study, Pelotas 2004 Birth Cohort

\begin{tabular}{|c|c|c|c|}
\hline Variables & $\begin{array}{l}\text { Included } \\
(n=3562)\end{array}$ & $\begin{array}{c}\text { Not included } \\
(n=669)\end{array}$ & $p$-value ${ }^{*}$ \\
\hline \multicolumn{4}{|l|}{ Maternal characteristics } \\
\hline Wealth index (quintiles) & & & 0.013 \\
\hline $1^{\text {st }}$ (lowest) to $3^{\text {st }}$ & $2107(59.1)$ & $430(64.3)$ & \\
\hline $4^{\text {th }}$ to $5^{\text {th }}$ (highest) & 1456 (40.9) & $238(35.7)$ & \\
\hline \multicolumn{3}{|l|}{ Maternal education (y) } & 0.007 \\
\hline$<5$ & $525(14.9)$ & $130(19.7)$ & \\
\hline $5-8$ & $1465(41.6)$ & $266(40.2)$ & \\
\hline$\geq 9$ & $1536(43.6)$ & $265(40.1)$ & \\
\hline \multicolumn{3}{|l|}{ Maternal age (y) } & 0.060 \\
\hline$\leq 19$ & $668(18.8)$ & $132(19.7)$ & \\
\hline $20-34$ & $2399(67.4)$ & $467(69.8)$ & \\
\hline$\geq 35$ & $493(13.9)$ & $70(10.5)$ & \\
\hline \multicolumn{3}{|l|}{ Marital status } & 0.001 \\
\hline Single/divorced/widowed & $556(15.6)$ & $139(20.8)$ & \\
\hline Married/living with a partner & 3006 (84.4) & $530(79.2)$ & \\
\hline \multicolumn{3}{|l|}{ Maternal skin color } & 0.956 \\
\hline White & $2602(73.1)$ & 488 (72.9) & \\
\hline Black/mixed & $960(26.9)$ & $181(27.1)$ & \\
\hline \multicolumn{3}{|l|}{ Parity } & 0.083 \\
\hline 0 & 1406 (39.5) & 260 (38.9) & \\
\hline 1 & $954(26.8)$ & $157(23.5)$ & \\
\hline$\geq 2$ & 1201 (33.7) & $252(37.7)$ & \\
\hline \multicolumn{3}{|l|}{ Mood symptoms during } & $<0.001$ \\
\hline pregnancy & $2706(76.0)$ & $463(69.2)$ & \\
\hline No & $854(24.0)$ & $206(30.8)$ & \\
\hline \multicolumn{4}{|l|}{ Yes } \\
\hline \multicolumn{3}{|l|}{ Smoking during pregnancy } & 0.008 \\
\hline No & 2612 (73.3) & 457 (68.3) & \\
\hline Yes & $950(26.7)$ & $212(31.7)$ & \\
\hline \multicolumn{3}{|l|}{ Alcohol during pregnancy } & 0.252 \\
\hline No & 3449 (96.8) & $642(96.0)$ & \\
\hline Yes & $113(3.2)$ & $27(4.0)$ & \\
\hline \multicolumn{4}{|l|}{ Children's characteristics } \\
\hline \multicolumn{3}{|l|}{ Gender } & 0.321 \\
\hline Male & $1837(51.6)$ & $359(53.7)$ & \\
\hline Female & $1725(48.4)$ & $310(46.3)$ & \\
\hline \multicolumn{3}{|l|}{ Multiple pregnancy } & 0.699 \\
\hline No & 3490 (98.0) & 657 (98.2) & \\
\hline Yes & $72(2.0)$ & $12(1.8)$ & \\
\hline \multirow{2}{*}{$\begin{array}{l}\text { 5-minute Apgar score } \\
<7\end{array}$} & & & $<0.001$ \\
\hline & 3488 (98.4) & 624 (94.5) & \\
\hline
\end{tabular}




\begin{tabular}{lccc}
$\geq 7$ & $56(1.6)$ & $36(5.5)$ & $<0.001$ \\
Low birthweight & & & \\
No & $3241(91.0)$ & $563(84.4)$ & \\
Yes & $320(9.0)$ & $104(15.6)$ & 0.006 \\
Preterm birth & & & \\
$\quad$ No & $3063(86.1)$ & $541(82.0)$ & \\
Yes & $494(13.9)$ & $119(18.0)$ & \\
\hline${ }^{*} x^{2}$ Test & & &
\end{tabular}


TABLE 2. Prevalence of Psychiatric Disorders among 11-year-olds, according to the DSM-5 and ICD-10 criteria, Pelotas 2004 Birth Cohort $(n=3,562)$

\begin{tabular}{|c|c|c|c|c|}
\hline \multirow[b]{2}{*}{ Psychiatric Disorder } & \multicolumn{2}{|r|}{ DSM-5 } & \multicolumn{2}{|r|}{ ICD-10 } \\
\hline & $\mathbf{N}$ & $\%(95 \% \mathrm{Cl})$ & $\mathbf{N}$ & $\%(95 \% \mathrm{Cl})$ \\
\hline Any psychiatric disorder & 471 & $13.2(12.1-14.4)$ & 401 & $11.3(10.2-12.3)$ \\
\hline Any anxiety disorder & 154 & $4.3(3.7-5.0)$ & 134 & $3.8(3.2-4.4)$ \\
\hline Separation anxiety & 41 & $1.2(0.8-1.6)$ & 22 & $0.6(0.4-0.9)$ \\
\hline Specific phobia & 63 & $1.8(1.4-2.3)$ & 62 & $1.7(1.3-2.2)$ \\
\hline Social phobia & 14 & $0.4(0.2-0.7)$ & 13 & $0.4(0.2-0.6)$ \\
\hline Panic disorder & 5 & $0.1(0.05-0.3)$ & 5 & $0.1(0.05-0.3)$ \\
\hline Agoraphobia & 7 & $0.2(0.1-0.4)$ & 7 & $0.2(0.08-0.4)$ \\
\hline Post-traumatic stress disorder & 8 & $0.2(0.1-0.4)$ & 7 & $0.2(0.08-0.4)$ \\
\hline Obsessive-compulsive disorder & 8 & $0.2(0.1-0.4)$ & 5 & $0.1(0.05-0.3)$ \\
\hline Body dysmorphic disorder & 8 & $0.2(0.1-0.4)$ & - & - \\
\hline Generalized anxiety disorder & 15 & $0.4(0.2-0.7)$ & 16 & $0.5(0.3-0.7)$ \\
\hline Other Anxiety disorder & 13 & $0.4(0.2-0.6)$ & 13 & $0.4(0.2-0.6)$ \\
\hline Any mood disorder & 115 & $3.2(2.7-3.9)$ & 42 & $1.2(0.9-1.6)$ \\
\hline Disruptive mood dysregulation disorder & 86 & $2.4(1.9-3.0)$ & - & - \\
\hline Major depressive disorder & 20 & $0.6(0.3-0.9)$ & - & - \\
\hline Other depressive disorder & 10 & $0.3(0.1-0.5)$ & 10 & $0.3(0.1-0.5)$ \\
\hline Mild depressive episode & - & - & 6 & $0.2(0.1-0.4)$ \\
\hline Moderate depressive episode & - & - & 13 & $0.4(0.2-0.6)$ \\
\hline Severe depressive episode & - & - & 4 & $0.1(0.03-0.3)$ \\
\hline Mania/bipolar disorder & 12 & $0.3(0.2-0.6)$ & 12 & $0.3(0.2-0.6)$ \\
\hline Any ADHD/ hyperactivity disorder & 144 & $4.0(3.4-4.7)$ & 120 & $3.4(2.8-4.0)$ \\
\hline
\end{tabular}


Combined-type ADHD

Inattentive-type ADHD

Hyperactive/impulsive-type ADHD

Hyperkinesia

Other Hyperactive/ Hyperkinetic disorder

Any conduct/oppositional disorder

Oppositional defiant disorder

Conduct disorder

Conduct disorder confined to family

Unsocialized conduct disorder

Socialized conduct disorder

Other Conduct/oppositional disorder

Any autism spectrum disorder

Autism spectrum disorder

Other autism spectrum disorder

Any tic disorder

Tourette syndrome

Chronic tic disorder

Other Tic disorder

Any eating disorder

Anorexia nervosa

Eating disorder not otherwise specified

Binge-eating disorder
$74 \quad 2.1(1.6-2.6)$

$35 \quad 1.0(0.7-1.4)$

$16 \quad 0.5(0.3-0.7)$

73

$2.1(1.6-2.6)$

19

$0.5(0.3-0.8) \quad 47$

$1.3(1.0-1.8)$

98

$2.8(2.2-3.3) \quad 118$

$3.3(2.7-4.0)$

68

$1.9(1.5-2.4) \quad 109$

$3.1(2.5-3.7)$

$35 \quad 1.0(0.7-1.4)$

$\begin{array}{cccc}- & - & 2 & 0.1(0.01-0.2) \\ - & - & 6 & 0.2(0.01-0.4) \\ - & - & 26 & 0.7(0.5-1.1) \\ 14 & 0.4(0.2-0.7) & 11 & 0.3(0.2-0.6) \\ 15 & 0.4(0.2-0.7) & 14 & 0.4(0.2-0.7) \\ 12 & 0.3(0.2-0.6) & 11 & 0.3(0.2-0.6) \\ 3 & 0.1(0.02-0.2) & 3 & 0.08(0.02-0.2) \\ 55 & 1.5(1.2-2.0) & 40 & 1.1(0.8-1.5) \\ 12 & 0.3(0.2-0.6) & 9 & 0.3(0.1-0.5) \\ 41 & 1.2(0.8-1.6) & 27 & 0.8(0.5-1.1) \\ 2 & 0.06(0.01-0.2) & 4 & 0.1(0.03-0.3) \\ 18 & 0.5(0.3-0.8) & 18 & 0.5(0.3-0.8) \\ 5 & 0.1(0.05-0.3) & 5 & 0.1(0.05-0.3) \\ 1 & 0.03(0.00-0.2) & 13 & 0.4(0.2-0.6) \\ 12 & 0.3(0.2-0.6) & - & -\end{array}$


TABLE 3. Prevalence of Psychiatric Disorders among 11-year-olds, by DSM-5 and ICD-10 categories, according to the adolescent sex, Pelotas 2004 Birth Cohort

DSM-5 CID-10

\begin{tabular}{|c|c|c|c|c|c|c|}
\hline \multirow[b]{3}{*}{ Category } & \multirow{2}{*}{\multicolumn{3}{|c|}{ Girls }} & \multirow{2}{*}{\multicolumn{2}{|c|}{ Boys $\quad$ Girls }} & \multirow[b]{3}{*}{ p-value* } \\
\hline & & & & & & \\
\hline & n (\%) & n (\%) & p-value* & n (\%) & n (\%) & \\
\hline Any psychiatric disorder & $287(15.6)$ & $184(10.7)$ & $<0.001$ & $247(13.5)$ & $154(8.9)$ & $<0.001$ \\
\hline Any anxiety disorder & $79(4.3)$ & $75(4.3)$ & 0.959 & $67(3.7)$ & $67(3.9)$ & 0.723 \\
\hline Any mood disorder & $69(3.8)$ & $46(2.7)$ & 0.064 & $24(1.3)$ & $18(1.0)$ & 0.462 \\
\hline Any ADHD/ hyperactivity disorder & $106(5.8)$ & $38(2.2)$ & $<0.001$ & $93(5.1)$ & $27(1.6)$ & $<0.001$ \\
\hline Any conduct/oppositional disorder & $69(3.8)$ & $29(1.7)$ & $<0.001$ & $83(4.5)$ & $35(2.0)$ & $<0.001$ \\
\hline Any autism spectrum disorder & $13(0.7)$ & $2(0.1)$ & 0.006 & $12(0.7)$ & $2(0.1)$ & 0.010 \\
\hline $\begin{array}{l}\text { Any tic disorder/Tourette } \\
\text { syndrome }\end{array}$ & $43(2.3)$ & $12(0.7)$ & $<0.001$ & $31(1.7)$ & $9(0.5)$ & 0.001 \\
\hline Any eating disorder & $5(0.3)$ & $13(0.8)$ & 0.043 & $5(0.3)$ & $13(0.8)$ & 0.043 \\
\hline
\end{tabular}

ADHD=attention deficit/hyperactivity disorder

${ }^{*}$ chi-square test 
TABLE 4. Prevalence of Psychiatric Disorders among 11-year-olds, by DSM-5 category according to the socioeconomic position (Wealth Index and maternal level of education), Pelotas 2004 Birth Cohort

Wealth Index (quintile), n (\%) Maternal Years of Schooling, $n(\%)$

\begin{tabular}{|c|c|c|c|c|c|c|c|c|c|c|}
\hline \multirow[b]{2}{*}{ Category } & & & & & & & & & & \\
\hline & 1 & 2 & 3 & 4 & 5 & $\mathbf{p}^{\star}$ & $<5$ & $5-8$ & $\geq 9$ & $\mathbf{p}^{\star}$ \\
\hline Any psychiatric disorder & $112(17.4)$ & $103(13.8)$ & $88(12.3)$ & $100(13.4)$ & $67(9.5)$ & $<0.001$ & $98(18.7)$ & 194 (13.2) & $173(11.3)$ & $<0.001$ \\
\hline Any anxiety disorder & $33(5.1)$ & $29(3.9)$ & $32(4.5)$ & $33(4.4)$ & $27(3.8)$ & 0.418 & $26(5.0)$ & $58(4.0)$ & $69(4.5)$ & 0.916 \\
\hline Any mood disorder & $27(4.2)$ & $34(4.6)$ & $21(2.9)$ & $21(2.8)$ & $12(1.7)$ & 0.001 & $36(6.9)$ & $53(3.6)$ & $25(1.6)$ & $<0.001$ \\
\hline Any ADHD/ hyperactivity disorder & $35(5.4)$ & $41(5.5)$ & $24(3.3)$ & $28(3.7)$ & $16(2.3)$ & 0.001 & $35(6.7)$ & $58(4.0)$ & $50(3.3)$ & 0.003 \\
\hline Any autism spectrum disorder & $3(0.5)$ & $3(0.4)$ & $2(0.3)$ & $6(0.8)$ & $1(0.1)$ & 0.773 & $2(0.4)$ & $8(0.6)$ & $5(0.3)$ & 0.634 \\
\hline Any tic disorder/Tourette syndrome & $12(1.9)$ & $9(1.2)$ & $13(1.8)$ & $8(1.1)$ & $13(1.8)$ & 0.920 & $7(1.3)$ & $24(1.6)$ & $23(1.5)$ & 0.922 \\
\hline Any eating disorder & $3(0.5)$ & $2(0.3)$ & $6(0.8)$ & $5(0.7)$ & $2(0.3)$ & 0.954 & $3(0.6)$ & $7(0.5)$ & $8(0.5)$ & 0.956 \\
\hline
\end{tabular}

ADHD=attention deficit/hyperactivity disorder

* chi-square test 
TABLE 5. Crude and adjusted analyses of the association between maternal and child's characteristics and any psychiatric disorder at age 11 according to DSM-5, Pelotas 2004 Birth Cohort

\begin{tabular}{|c|c|c|c|c|}
\hline \multirow[t]{2}{*}{ Variable } & \multicolumn{2}{|c|}{ Crude analysis } & \multicolumn{2}{|c|}{ Adjusted analysis } \\
\hline & OR $(95 \% \mathrm{Cl})$ & p-value & OR $(95 \% \mathrm{Cl})$ & p-value \\
\hline \multicolumn{5}{|c|}{ Level 1 - demographic and socioeconomic variables } \\
\hline Wealth index (quintiles) & & $<0.001$ & & 0.152 \\
\hline $1^{\text {st }}$ (lowest) & $2.0(1.5 ; 2.8)$ & & $1.6(1.1 ; 2.3)$ & \\
\hline $2^{\text {nd }}$ & $1.5(1.1 ; 2.1)$ & & $1.3(0.9 ; 1.9)$ & \\
\hline $3^{\text {rd }}$ & $1.3(1.0 ; 1.9)$ & & $1.2(0.8 ; 1.7)$ & \\
\hline $4^{\text {th }}$ & $1.5(1.1 ; 2.0)$ & & $1.4(1.0 ; 2.0)$ & \\
\hline $5^{\text {th }}$ (highest) & 1.0 (Reference) & & 1.0 (Reference) & \\
\hline Maternal education (y) & & $<0.001$ & & 0.042 \\
\hline$<5$ & $1.8(1.4 ; 2.4)$ & & $1.4(1.0 ; 1.9)$ & \\
\hline $5-8$ & $1.2(1.0 ; 1.5)$ & & $1.0(0.8 ; 1.3)$ & \\
\hline$\geq 9$ & 1.0 (Reference) & & 1.0 (Reference) & \\
\hline Maternal age $(y)$ & & 0.090 & & 0.128 \\
\hline$\leq 19$ & $1.2(0.9 ; 1.5)$ & & $1.2(0.9 ; 1.6)$ & \\
\hline $20-34$ & 1.0 (Reference) & & 1.0 (Reference) & \\
\hline$\geq 35$ & $0.8(0.6 ; 1.1)$ & & $0.8(0.6 ; 1.1)$ & \\
\hline Marital status & & 0.051 & & 0.121 \\
\hline $\begin{array}{l}\text { Married/living with a } \\
\text { partner }\end{array}$ & $\begin{array}{c}1.0 \text { (Reference) } \\
1.3(1.0: 1.7)\end{array}$ & & $\begin{array}{c}1.0 \text { (Reference) } \\
1.2(0.9: 1.6)\end{array}$ & \\
\hline Single/divorced/widowed & & & & \\
\hline Maternal skin color & & 0.072 & & 0.507 \\
\hline White & 1.0 (Reference) & & 1.0 (Reference) & \\
\hline Black/mixed & $1.2(0.9 ; 1.5)$ & & $1.1(0.9 ; 1.3)$ & \\
\hline Parity & & 0.020 & & 0.064 \\
\hline 0 & $1.2(0.9 ; 1.5)$ & & $1.1(0.8 ; 1.4)$ & \\
\hline 1 & 1.0 (Reference) & & 1.0 (Reference) & \\
\hline$\geq 2$ & $1.4(1.1 ; 1.8)$ & & $1.4(1.0 ; 1.8)$ & \\
\hline \multicolumn{5}{|c|}{ Level 2 - variables related to maternal behavioral traits } \\
\hline Smoking during pregnancy & & $<0.001$ & & 0.021 \\
\hline No & 1.0 (Reference) & & 1.0 (Reference) & \\
\hline Yes & $1.5(1.2 ; 1.9)$ & & $1.3(1.0 ; 1.6)$ & \\
\hline Alcohol during pregnancy & & 0.058 & & 0.139 \\
\hline No & 1.0 (Reference) & & 1.0 (Reference) & \\
\hline Yes & $1.6(1.0 ; 2.6)$ & & $1.4(0.9 ; 2.4)$ & \\
\hline \multicolumn{2}{|c|}{ Level 3 - Moods symptoms during pregnancy } & & & \\
\hline $\begin{array}{l}\text { Mood symptoms during } \\
\text { preanancy }\end{array}$ & & $<0.001$ & & $<0.001$ \\
\hline No & 1.0 (Reference) & & 1.0 (Reference) & \\
\hline Yes & $1.5(1.2 ; 1.9)$ & & $1.4(1.1 ; 1.7)$ & \\
\hline \multicolumn{5}{|c|}{ Level 4 - Child's characteristics at birth } \\
\hline Gender & & $<0.001$ & & $<0.001$ \\
\hline Male & 1.0 (Reference) & & 1.0 (Reference) & \\
\hline Female & $0.6(0.5 ; 0.8)$ & & $0.6(0.5 ; 0.8)$ & \\
\hline Multiple pregnancy & & 0.859 & & 0.606 \\
\hline No & 1.0 (Reference) & & 1.0 (Reference) & \\
\hline
\end{tabular}




\begin{tabular}{|c|c|c|c|c|}
\hline Yes & $0.9(0.5 ; 1.9)$ & & $0.8(0.4 ; 1.7)$ & \\
\hline 5-minute Apgar score & & 0.010 & & 0.028 \\
\hline$\geq 7$ & 1.0 (Reference) & & 1.0 (Reference) & \\
\hline$<7$ & $2.2(1.2 ; 4.1)$ & & $2.1(1.1 ; 3.9)$ & \\
\hline Low birthweight & & 0.097 & & 0.620 \\
\hline No & 1.0 (Reference) & & 1.0 (Reference) & \\
\hline Yes & $1.3(1.0 ; 1.8)$ & & $1.1(0.8 ; 1.6)$ & \\
\hline Preterm birth & & 0.009 & & 0.044 \\
\hline No & 1.0 (Reference) & & 1.0 (Reference) & \\
\hline Yes & $1.4(1.1 ; 1.8)$ & & $1.3(1.0 ; 1.7)$ & \\
\hline \multicolumn{5}{|c|}{$\begin{array}{l}\text { Level } 5 \text { - Trajectories of maternal depressive symptoms in the first years of the adolescents' } \\
\text { life }\end{array}$} \\
\hline $\begin{array}{l}\text { Trajectories of maternal } \\
\text { depressive symptoms }\end{array}$ & & $<0.001$ & & $<0.001$ \\
\hline Low & 1.0 (Reference) & & 1.0 (Reference) & \\
\hline Moderate -low & $1.9(1.4 ; 2.5)$ & & $1.7(1.3 ; 2.4)$ & \\
\hline Increasing & $3.9(2.7 ; 5.6)$ & & $3.4(2.3 ; 5.1)$ & \\
\hline Decreasing & $3.9(2.7 ; 5.6)$ & & $3.5(2.4 ; 5.2)$ & \\
\hline High-chronic & $5.3(3.5 ; 8.1)$ & & $4.7(3.0 ; 7.5)$ & \\
\hline
\end{tabular}


"Agir, eis a inteligência verdadeira.

Serei o que quiser. Mas tenho que querer o que for. O êxito está em ter êxito, e não em ter condições de êxito. Condições de palácio tem qualquer terra larga, mas onde estará o palácio se não o fizerem ali?" 
7. ARTIGO 2 - CONTINUIDADE DOS TRANSTORNOS MENTAIS ENTRE OS 6 E 11 ANOS: COORTE DE PELOTAS DE 2004

Este capítulo apresenta o artigo "Continuity of psychiatric disorders between 6 and 11 years of age: the 2004 Pelotas Birth Cohort" de autoria de Carolina La Maison, Tiago N Munhoz, Iná S. Santos, Aluísio J. Barros, Mariana Amaral, Luciana Anselmi, Fernando C. Barros, Maria A. PastorValero, Alicia Matijasevich.

O artigo foi originalmente submetido à revista BMC Psychiatryc e aguarda o processo de avaliação. Sendo assim, apresenta-se a seguir os resultados e a discussão deste produto, finalizando os objetivos da tese. 


\subsection{Results}

\subsubsection{Attrition analyses}

Of the 4231 participants constituting the original cohort, 98 died in the first eleven years of life. At ages 6 and 11, we interviewed 3721 and 3566 individuals, respectively. Data on SDQ scores at both ages were available for 3362 individuals $(79.5 \%$ of the original cohort). Among the members not included in the analysis, those belonging to families with a higher socioeconomic status, those born to 20-34 year-old mothers and those born with low birth weight were overrepresented (Table 1).

\subsubsection{Sample description}

The individuals under study and their mothers are described in Table 1. Approximately $70 \%$ of the families earned $\leq 3$ times the minimum wage, and $15 \%$ of the mothers had $\leq 4$ years of schooling. Of the mothers, $73 \%$ were White; $40 \%$ were primiparous; $67 \%$ were between 20 and 34 years of age; and $73 \%$ did not smoke during pregnancy. There was a slight predominance of boys, who accounted for $52 \%$ of the sample, and $9 \%$ of the cohort members were born with low birth weight.

\subsubsection{Prevalence, incidence and persistence of mental health problems}

The prevalence of "any SDQ difficulty" was found to be $10.4 \%$ and $14.2 \%$ at ages 6 and 11, respectively, translating to an increase of $50 \%$ between the two time points (Table 2). From 6 years of age to 11 years of age, there was an increase in the prevalence of emotional symptoms and hyperactivity/inattention, whereas there was a decrease in the prevalence of 
conduct problems. In the analysis stratified by sex, the prevalence of "any SDQ difficulty" was higher in boys than in girls, at both ages (see Additional file 1: Table S1). Among the girls, there were no changes from age 6 to age 11 in terms of conduct problems, hyperactivity/inattention or peer relationship problems.

The overall incidence of "any difficulty" over the study period was 10.6\% (Table 2), being $12.7 \%$ in boys and $8.4 \%$ in girls (see Additional file 1 : Table S1). The overall incidence of emotional symptoms was higher than was that of the other problems investigated. The incidence of conduct problems was similar (approximately 9\%) between boys and girls, whereas hyperactivity/inattention and peer relationship problems had both higher incidence among boys than among girls (see Additional file 1: Table S1).

Over the study period, the persistence of "any SDQ difficulty" in the total population was $45 \%$. Emotional symptoms, hyperactivity/inattention and peer relationship problems persisted in about $40-45 \%$ of the sample, whereas conduct problems persisted in only $33.3 \%$ (Table 2 ). The persistence rate of hyperactivity/inattention was higher among boys than among girls (55\% vs. $31 \%)$.

\subsubsection{Homotypic and heterotypic continuity}

From age 6 to age 11, there was significant homotypic continuity for emotional symptoms, conduct problems, hyperactivity/inattention and peer relationship problems (Table 3), with no significant difference between boys and girls (see Additional file 2: Table S2). There was also heterotypic continuity for all four of those outcomes. After adjusted analysis for comorbidity at age 6 , emotional symptoms and hyperactivity/inattention during 
childhood were found to be associated to conduct and peer relationship problems at age 11. Exhibiting conduct problems at 6 years of age was found to be associated to the occurrence of hyperactivity/inattention and peer relationship problems at 11 years of age. Peer relationship problems at age 6 were associated to emotional symptoms, conduct problems and hyperactivity/inattention in early adolescence (Table 3). Hyperactivity disorders in childhood were associated with conduct disorders at age 11 in both sexes. For the emotional symptoms, conduct problems and peer relationship problems sub-scales, there were sex-related differences in the patterns of heterotypic continuity (see Additional file 2: Table S2).

\subsection{Discussion}

The prevalence of "any SDQ difficulty" increased by 50\% between 6 and 11 years of age with the prevalence of psychiatric disorders for both ages, being higher in boys than in girls. From age 6 to age 11, the prevalence of emotional symptoms increased by $50 \%$, whereas that of conduct problems declined by $12 \%$. During the study period as a whole, the overall incidence and the persistence of "any SDQ difficulty" was $10.6 \%$ and $45.0 \%$, respectively. During the transition from childhood to early adolescence, homotypic continuity was observed for emotional symptoms, conduct problems, hyperactivity/inattention and peer relationship problems. For emotional symptoms, conduct problems and peer relationship problems, the patterns of heterotypic continuity differed between girls and boys.

In the present study, we observed an increase in the prevalence of psychiatric disorders, especially emotional symptoms and 
hyperactivity/inattention, from age 6 to age 11. A recent review reported changes in the prevalence of psychiatric disorders from childhood to adolescence (Costello et al., 2011). In that review, the largest epidemiological study that covered childhood and early adolescence was the British Child and Adolescent Mental Health Survey, conducted in 1999 and involving over 10,000 children aged 5-15 years. Rates of any psychiatric disorder (DSM-IV criteria) rose from $8.6 \%$ at $8-10$ years of age to $9.6 \%$ at $11-12$ years of age. Similar to what was observed in our study, that review showed increased rates for most internalizing disorders as children moved into adolescence. However, contrary to our results, most of the studies included in the review showed decreasing rates of attention-deficit/hyperactivity disorder (ADHD). The results of trend analyses for conduct disorder and oppositional defiant disorder were mixed, increasing rates of conduct disorder and decreasing rates of oppositional defiant disorder being reported in the British Child and Adolescent Mental Health Survey, whereas results were divergent across the other studies included.

Anxiety disorders are the most common psychiatric disorders in children and adolescents, and it is known that their incidence increases during adolescence and adulthood, as does that of depressive disorders (Compas \& Oppedisano, 2000) (Zahn-Waxler, Shirtcliff, \& Marceau, 2008). Specifically in relation to childhood and adolescence, there is evidence that boys initially present a higher prevalence of depressive disorders than do girls, although that pattern tends to reverse between 11 and 13 years of age (yranowski, Frank, Young, \& Shear, 2000). Those differences are associated with a multifactorial aetiology, including biological, psychosocial and socio-economic 
aspects (Salk, Hyde, \& Abramson, 2017). The pattern of occurrence of internalizing symptoms during childhood and adolescence can be explained from a perspective of multilevel developmental psychopathology (Shanahan, Calkins, Keane, Kelleher, \& Suffness, 2014). In keeping with the findings of the present study, previous investigations evaluating internalizing symptoms in children aged 2-10 years have reported only small differences between boys and girls in terms of the occurrence of symptoms (Colder, Mott, \& Berman, 2002; Gazelle \& Ladd, 2003; Sterba, Prinstein, \& Cox, 2007). Factors related to temperament and to the quality of interpersonal relationships have been shown to be major predictors of such disorders in children and adolescents (Gazelle \& Ladd, 2003). A lifetime history of exposure to stressors has been associated with an increase in the occurrence of depressive symptoms, especially in girls (Thapar, Collishaw, Pine, \& Thapar, 2012).

The combined prevalence of ADHD in children under 18 years of age is 5\% (G. Polanczyk, de Lima, Horta, Biederman, \& Rohde, 2007). The symptomatology that accompanies these disorders affects all aspects of the lives of children and adolescents, as well as those of their parents and siblings, thus impairing family and marital functioning (ㅂarpin, 2005). In our sample, we observed an increase in hyperactivity/inattention between the ages of 6 and 11 years. That could be attributable to the fact that the symptoms of hyperactivity are more harmful and easier to recognize in that they magnify academic challenges and make behavioural control essential to achieving educational goals. 
Our results are consistent with those of previous studies evaluating individuals in similar age groups, in which psychiatric disorders have been found to show homotypic and heterotypic continuity. Shevlin et al. evaluated 4815 individuals aged $7.5-14$ years who were enrolled in an English birth cohort study (the Avon Longitudinal Study of Parents and Children) and observed high rates of homotypic and heterotypic continuity (Shevlin, McElroy, \& Murphy, 2017). A study of 1584 adolescents reported continuity of psychiatric disorders between the ages of 11 and 19 years, mainly due to the recurrence of mood disorders and chronicity of anxiety disorders (Ormel et al., 2015). In that study, there was stronger evidence of continuity for externalizing disorders (especially ADHD) than for internalizing disorders such as depression and anxiety. In the United States, Costello et al. evaluated homotypic and heterotypic continuity in 1420 individuals between 9 and 16 years of age who were enrolled in the Great Smoky Mountains Study (Costello et al., 2003). The authors found that the presence of any psychiatric diagnosis in childhood increased the chance of presenting any psychiatric disorder in adolescence, the risk being greater among the girls than among the boys. In general, homotypic continuity, especially for depressive and anxiety disorders (generalized anxiety, social phobia and specific phobia), was more common among the girls in their study. The authors also observed heterotypic continuity for internalizing disorders (anxiety and depressive disorders) and externalizing disorders (ADHD and conduct disorder) (ostello et al., 2003), similar to what was observed in the present study. In a recent study involving children and adolescents aged 9-11 years, conducted at a total of 73 primary schools in London, the persistence rates of internalizing 
and externalizing symptoms were found to be approximately $17 \%$ over the two years between the baseline and follow-up assessments (Lancefield, Raudino, Downs, \& Laurens, 2016). In Brazil, a study analyzing a sample of 601 children, all of whom were members of the 1993 Pelotas Birth Cohort, showed that, approximately in $30 \%$ of the children, the conduct problems and emotional symptoms observed at 4 years of age persisted at 12 years of age (Anselmi et al., 2008). In the present study, similar to what was observed by Wichstrom et al., early behavioural disorders increased the chance of later symptoms of hyperactivity, whereas early symptoms of hyperactivity increased the chance of later emotional symptoms and behavioural disorders (Wichstrom, Belsky, \& Steinsbekk, 2017).

The patterns of heterotypic continuity observed in the present study are in line with the most current concept of psychopathology, as a phenomenon subject to changes in its manifestations and phenotypic plasticity (NolenHoeksema \& Watkins, 2011). Two hypotheses have been proposed to explain the development of psychiatric disorders: that of shared aetiology; and that of causal interaction. The shared aetiology hypothesis suggests that the persistence of risk factors could explain the co-occurrence and continuity of psychiatric disorders over time. For example, genetic factors could predispose individuals to certain psychiatric disorders, the manifestations of which could appear over time, possibly reflecting changes in environmental factors (Lahey, Zald, Hakes, Krueger, \& Rathouz, 2014). This shared aetiology hypothesis was also supported by Caspi et al. (Caspi et al., 2014). An alternative interpretation of heterotypic continuity establishes causal interactions among psychopathological comorbidities. The causal interaction hypothesis suggests 
that, within a complex network, psychiatric symptoms/disorders have the potential to influence each another, directly and indirectly, over time ( $\underline{\text { Cramer, }}$ Waldorp, van der Maas, \& Borsboom, 2010).

One of the strengths of our study is that it was a population-based study, involving face-to-face interviews and a large sample of enrolees. In addition, we assessed mental health problems with an internationally recognized screening tool that has been validated for use in Brazil and was applied by psychologists who had been trained to ensure the quality of the data. We also emphasize the low proportion of non-respondents. There were few differences between the individuals who were included in our analysis and those who were lost to follow-up, suggesting that any attrition bias is likely to be small.

Our study has some limitations. We applied the parent-report version of the SDQ, which is only one of the three versions available. The application of the teacher-report and self-report versions of the SDQ might have revealed other symptoms not recognized by mothers or caregivers, contributing to a more accurate screening for psychiatric disorders in the population studied.

\subsection{REFERENCES}

Anselmi, L., Barros, F. C., Teodoro, M. L., Piccinini, C. A., Menezes, A. M., Araujo, C. L., \& Rohde, L. A. (2008). Continuity of behavioral and emotional problems from pre-school years to pre-adolescence in a developing country. $J$ Child Psychol Psychiatry, 49(5), 499-507. doi: 10.1111/j.1469-

7610.2007.01865.x 
Caspi, A., Houts, R. M., Belsky, D. W., Goldman-Mellor, S. J., Harrington, H., Israel, S., . . . Moffitt, T. E. (2014). The p Factor: One General Psychopathology Factor in the Structure of Psychiatric Disorders? Clin Psychol Sci, 2(2), 119-137. doi: 10.1177/2167702613497473

Colder, C. R., Mott, J. A., \& Berman, A. S. (2002). The interactive effects of infant activity level and fear on growth trajectories of early childhood behavior problems. Dev Psychopathol, 14(1), 1-23.

Compas, B. E., \& Oppedisano, G. (2000). Mixed anxiety/depression in childhood and adolescence. In A. J. Sameroff, M. Lewis \& S. Miller (Eds.), Handbook of developmental psychopathology (2nd ed., pp. 531-548). Boston, MA: Springer.

Costello, E. J., Copeland, W., \& Angold, A. (2011). Trends in psychopathology across the adolescent years: what changes when children become adolescents, and when adolescents become adults? J Child Psychol Psychiatry, 52(10), 1015-1025. doi: 10.1111/j.1469-7610.2011.02446.x

Costello, E. J., Mustillo, S., Erkanli, A., Keeler, G., \& Angold, A. (2003). Prevalence and development of psychiatric disorders in childhood and adolescence. Arch Gen Psychiatry, 60(8), 837-844. doi: 10.1001/archpsyc.60.8.837

Cramer, A. O., Waldorp, L. J., van der Maas, H. L., \& Borsboom, D. (2010). Comorbidity: a network perspective. Behav Brain Sci, 33(2-3), 137-150; discussion 150-193. doi: 10.1017/S0140525X09991567

Cyranowski, J. M., Frank, E., Young, E., \& Shear, M. K. (2000). Adolescent onset of the gender difference in lifetime rates of major depression: a theoretical model. Arch Gen Psychiatry, 57(1), 21-27. 
Gazelle, H., \& Ladd, G. W. (2003). Anxious solitude and peer exclusion: a diathesis-stress model of internalizing trajectories in childhood. Child Dev, 74(1), 257-278.

Harpin, V. A. (2005). The effect of ADHD on the life of an individual, their family, and community from preschool to adult life. Arch Dis Child, 90 Suppl 1, i2-7. doi: 10.1136/adc.2004.059006

Lahey, B. B., Zald, D. H., Hakes, J. K., Krueger, R. F., \& Rathouz, P. J. (2014). Patterns of heterotypic continuity associated with the cross-sectional correlational structure of prevalent mental disorders in adults. JAMA Psychiatry, 71(9), 989-996. doi: 10.1001/jamapsychiatry.2014.359

Lancefield, K. S., Raudino, A., Downs, J. M., \& Laurens, K. R. (2016). Trajectories of childhood internalizing and externalizing psychopathology and psychotic-like experiences in adolescence: A prospective population-based cohort study. Dev Psychopathol, 28(2), 527-536. doi: $10.1017 / \mathrm{s} 0954579415001108$

Nolen-Hoeksema, S., \& Watkins, E. R. (2011). A Heuristic for Developing Transdiagnostic Models of Psychopathology: Explaining Multifinality and Divergent Trajectories. Perspect Psychol Sci, 6(6), 589-609. doi: $10.1177 / 1745691611419672$

Ormel, J., Raven, D., van Oort, F., Hartman, C. A., Reijneveld, S. A., Veenstra, R., . . . Oldehinkel, A. J. (2015). Mental health in Dutch adolescents: a TRAILS report on prevalence, severity, age of onset, continuity and co-morbidity of DSM disorders. Psychol Med, 45(2), 345-360. doi: $10.1017 / S 0033291714001469$ 
Polanczyk, G., de Lima, M. S., Horta, B. L., Biederman, J., \& Rohde, L. A. (2007). The worldwide prevalence of ADHD: a systematic review and metaregression analysis. Am J Psychiatry, 164(6), 942-948. doi: 10.1176/ajp.2007.164.6.942

Salk, R. H., Hyde, J. S., \& Abramson, L. Y. (2017). Gender differences in depression in representative national samples: Meta-analyses of diagnoses and symptoms. Psychol Bull, 143(8), 783-822. doi: 10.1037/bul0000102 Shanahan, L., Calkins, S. D., Keane, S. P., Kelleher, R., \& Suffness, R. (2014). Trajectories of internalizing symptoms across childhood: The roles of biological self-regulation and maternal psychopathology. Dev Psychopathol, 26(4 Pt 2), 1353-1368. doi: 10.1017/S0954579414001072

Shevlin, M., McElroy, E., \& Murphy, J. (2017). Homotypic and heterotypic psychopathological continuity: a child cohort study. Soc Psychiatry Psychiatr Epidemiol, 52(9), 1135-1145. doi: 10.1007/s00127-017-1396-7

Sterba, S. K., Prinstein, M. J., \& Cox, M. J. (2007). Trajectories of internalizing problems across childhood: heterogeneity, external validity, and gender differences. Dev Psychopathol, 19(2), 345-366. doi: $10.1017 / \mathrm{s} 0954579407070174$

Thapar, A., Collishaw, S., Pine, D. S., \& Thapar, A. K. (2012). Depression in adolescence. Lancet, 379(9820), 1056-1067. doi: 10.1016/S0140$6736(11) 60871-4$

Wichstrom, L., Belsky, J., \& Steinsbekk, S. (2017). Homotypic and heterotypic continuity of symptoms of psychiatric disorders from age 4 to 10 years: a dynamic panel model. J Child Psychol Psychiatry, 58(11), 1239-1247. doi: $10.1111 /$ jcpp. 12754 
Zahn-Waxler, C., Shirtcliff, E. A., \& Marceau, K. (2008). Disorders of childhood and adolescence: gender and psychopathology. Annu Rev Clin Psychol, 4, 275-303. doi: 10.1146/annurev.clinpsy.3.022806.091358 
Table 1 Baseline characteristics of the mothers and infants included and not included in the study

\begin{tabular}{|c|c|c|c|}
\hline Variables & $\begin{array}{l}\text { Included } \\
(n=3362)\end{array}$ & $\begin{array}{l}\text { Not included } \\
(n=869)\end{array}$ & $p$-value ${ }^{*}$ \\
\hline $\begin{array}{l}\text { Monthly family income (minimum } \\
\text { wage), } n(\%)\end{array}$ & & & 0.005 \\
\hline$\leq 3.0$ & $2246(67.0)$ & $577(66.8)$ & \\
\hline $3.1-6.0$ & $768(22.9)$ & $176(20.4)$ & \\
\hline $6.1-10.0$ & $185(5.5)$ & $58(6.7)$ & \\
\hline$>10.0$ & $154(4.6)$ & $53(6.1)$ & \\
\hline Maternal schooling (years), $n(\%)$ & & & 0.059 \\
\hline$\leq 4$ & $499(15.0)$ & $156(18.1)$ & \\
\hline $5-8$ & $1395(41.9)$ & $336(39.1)$ & \\
\hline$\geq 9$ & $1433(43.1)$ & $368(42.8)$ & \\
\hline Maternal age (years), $n(\%)$ & & & 0.005 \\
\hline$\leq 19$ & $640(19.0)$ & $160(18.5)$ & \\
\hline $20-34$ & $2246(66.8)$ & $619(71.4)$ & \\
\hline$\geq 35$ & $475(14.1)$ & $88(10.2)$ & \\
\hline Maternal skin colour, $n(\%)$ & & & 0.792 \\
\hline White & $2459(73.1)$ & $631(72.7)$ & \\
\hline Black/other & $903(26.9)$ & $237(27.3)$ & \\
\hline Parity, $n(\%)$ & & & 0.109 \\
\hline 0 & $1329(39.5)$ & $337(38.8)$ & \\
\hline 1 & $897(26.7)$ & $214(24.6)$ & \\
\hline 2 & 545 (16.2) & $135(15.5)$ & \\
\hline$\geq 3$ & $590(17.6)$ & $183(21.1)$ & \\
\hline Smoking during pregnancy, $n(\%)$ & & & 0.069 \\
\hline No & $2460(73.2)$ & $609(70.1)$ & \\
\hline Yes & $902(26.8)$ & $260(29.9)$ & \\
\hline Sex of the infant, $n(\%)$ & & & 0.308 \\
\hline Male & $1732(51.5)$ & $464(53.5)$ & \\
\hline Female & $1630(48.5)$ & $404(46.5)$ & \\
\hline Low birthweight $(<2500 \mathrm{~g}), n(\%)$ & & & $<0.001$ \\
\hline No & $3062(91.1)$ & $741(85.6)$ & \\
\hline Yes & $299(8.9)$ & $125(14.4)$ & \\
\hline
\end{tabular}

${ }^{*}$ chi-square test. 
Table 2 Prevalence, incidence and persistence rate for any SDQ difficulty, emotional symptoms, conduct problems, hyperactivity/inattention and peer relationship problems

\begin{tabular}{|c|c|c|c|c|c|c|c|}
\hline \multirow[t]{2}{*}{ Disorder } & \multicolumn{2}{|c|}{ Prevalence rate at age 6} & \multicolumn{2}{|c|}{ Prevalence rate at age 11} & \multirow[t]{2}{*}{$p$-value* } & \multirow{2}{*}{$\begin{array}{l}\text { Cumulative } \\
\text { incidence from age } \\
6 \text { to age } 11^{\star \star} \\
\%(95 \% \mathrm{Cl})\end{array}$} & \multirow{2}{*}{$\begin{array}{l}\text { Persistence between } \\
\text { age } 6 \text { and age } 11^{\star * *} \\
\%(95 \% \mathrm{Cl})\end{array}$} \\
\hline & $N$ & $\%(95 \% \mathrm{Cl})$ & $N$ & $\%(95 \% \mathrm{Cl})$ & & & \\
\hline Any SDQ difficulty & 350 & $10.4(9.4-11.5)$ & 476 & $14.2(13.0-15.4)$ & $<0.001$ & $10.6(9.5-11.7)$ & $44.9(39.6-50.2)$ \\
\hline Emotional symptoms & 449 & $13.4(12.2-14.6)$ & 672 & $20.0(18.6-21.4)$ & $<0.001$ & $16.3(15.0-17.7)$ & $43.9(39.2-48.6)$ \\
\hline Conduct problems & 492 & $14.6(13.5-15.9)$ & 433 & $12.9(11.8-14.1)$ & 0.018 & $9.4(8.3-10.5)$ & $33.3(29.2-37.7)$ \\
\hline Hyperactivity/inattention & 455 & $13.5(12.4-14.7)$ & 535 & $15.9(14.7-17.2)$ & 0.001 & $11.3(10.2-12.5)$ & $45.5(40.9-50.2)$ \\
\hline Peer relationship & 466 & $13.9(12.7-15.1)$ & 450 & $13.4(12.3-14.6)$ & 0.526 & $9.4(8.3-10.5)$ & $38.4(34.0-43.0)$ \\
\hline
\end{tabular}

Note: $\mathrm{SDQ}=$ Strengths and Difficulties Questionnaire; $\mathrm{Cl}=$ confidence interval

${ }^{*}$ McNemar test for the difference in prevalence rates between age 6 and age 11

**The cumulative incidence of any SDQ difficulty between 6 and 11 years of age was calculated as the proportion of children with any SDQ difficulty at 11 years amongst all those who had tested positive at 6 years. The same procedure was used in order to calculate cumulative incidence of emotional symptoms, conduct problems, hyperactivity/inattention and peer relationship problems. ${ }^{* * *}$ The persistence of any SDQ difficulty between 6 and 11 years of age was calculated as the proportion of children with any SDQ difficulty at 11 years amongst all those who had tested positive at age 6 . The same procedure was used in order to calculate persistence of emotional symptoms, conduct problems, hyperactivity/inattention and peer relationship problems. 
Table 3 Logistic regression analyses of disorders at age 6 as predictors of disorders at age $11^{\text {a }}$

\begin{tabular}{|c|c|c|c|c|c|c|c|c|}
\hline \multirow[t]{2}{*}{ Assessment at age 6} & \multicolumn{8}{|c|}{ Assessment at age 11} \\
\hline & OR $(95 \% \mathrm{Cl})$ & $p$-Value & OR $(95 \% \mathrm{Cl})$ & $p$-Value & OR $(95 \% \mathrm{Cl})$ & $p$-Value & OR $(95 \% \mathrm{Cl})$ & $p$-Value \\
\hline Emotional symptoms & $4.0(3.3-5.0)$ & $=0.001$ & $2.3(1.8-3.0)$ & $<0.001$ & $2.1(1.7-2.7)$ & $<0.001$ & $3.2(2.5-4.1)$ & $<0.001$ \\
\hline Conduct problems & $1.5(1.2-1.9)$ & $=0.001$ & $4.8(3.9-6.1)$ & $<0.001$ & $3.4(2.7-4.2)$ & $<0.001$ & $2.7(2.1-3.4)$ & $<0.001$ \\
\hline+ comorbidity $^{\mathrm{b}}$ & $0.9(0.7-1.1)$ & 0.317 & & & $1.6(1.3-2.1)$ & $<0.001$ & $1.4(1.1-1.9)$ & 0.018 \\
\hline Hyperactivity/inattention & $1.7(1.4-2.2)$ & $=0.001$ & $3.5(2.8-4.4)$ & $<0.001$ & $6.6(5.3-8.2)$ & $<0.001$ & $2.4(1.9-3.0)$ & $<0.001$ \\
\hline
\end{tabular}

Note: $\mathrm{OR}=$ odds ratio; $\mathrm{Cl}=$ confidence interval

${ }^{a} n=3362$ individuals with data available for evaluations at age 6 and age 11

${ }^{b}$ Analyses conducted to control for concurrent age 6 comorbidity in the heterotypic analyses 
Table S1 Prevalence, cumulative incidence and persistence of problems between ages 6 and 11, by sex

\begin{tabular}{|c|c|c|c|c|c|c|c|}
\hline \multirow[t]{2}{*}{ Disorder } & \multicolumn{2}{|c|}{ Prevalence rate at age 6} & \multicolumn{2}{|c|}{ Prevalence rate at age 11} & \multirow[t]{2}{*}{$p$-value* } & \multirow{2}{*}{$\begin{array}{l}\text { Cumulative incidence } \\
\text { between age } 6 \text { and age } 11^{\star *} \\
\%(95 \% \mathrm{Cl})\end{array}$} & \multirow{2}{*}{$\begin{array}{l}\text { Persistence between } \\
\text { age } 6 \text { and age } 11^{* * *} \\
\%(95 \% \mathrm{Cl})\end{array}$} \\
\hline & $n$ & $\%(95 \% \mathrm{Cl})$ & $N$ & $\%(95 \% \mathrm{Cl})$ & & & \\
\hline \multicolumn{8}{|l|}{ Male $(n=1732)$} \\
\hline Total difficulties & 201 & $11.6(10.1-13.2)$ & 285 & $16.5(14.7-18.3)$ & $<0.001$ & $12.7(11.0-14.4)$ & $45.3(38.3-52.4)$ \\
\hline Emotional symptoms & 222 & $12.8(11.3-14.5)$ & 328 & $18.9(17.1-20.9)$ & $<0.001$ & $15.4(13.7-17.4)$ & $42.8(36.2-50.0)$ \\
\hline Conduct problems & 282 & $16.3(14.6-18.1)$ & 242 & $14.0(12.4-15.7)$ & 0.029 & $9.7(8.2-11.3)$ & $36.2(30.6-42.1)$ \\
\hline Hyperactivity/inattention & 275 & $15.9(14.2-17.7)$ & 356 & $20.6(18.7-22.5)$ & $<0.001$ & $14.1(12.3-16.0)$ & $54.9(48.8-60.9)$ \\
\hline $\begin{array}{l}\text { Peer relationship } \\
\text { problems }\end{array}$ & 264 & $15.2(13.6-17.0)$ & 268 & $15.5(13.8-17.3)$ & 0.866 & $11.0(9.4-12.7)$ & $40.5(34.6-46.7)$ \\
\hline \multicolumn{8}{|l|}{ Female $(n=1630)$} \\
\hline Total difficulties & 149 & $9.1(7.8-10.6)$ & 191 & $11.7(10.2-13.4)$ & 0.004 & $8.4(7.1-10.0)$ & $44.3(36.2-52.7)$ \\
\hline Emotional symptoms & 227 & $13.9(12.3-15.7)$ & 344 & $21.1(19.1-23.2)$ & $<0.001$ & $17.2(15.3-19.3)$ & $44.9(38.3-51.7)$ \\
\hline Conduct problems & 210 & $12.9(11.3-14.6)$ & 191 & $11.7(10.2-13.4)$ & 0.279 & $9.1(7.6-10.7)$ & $29.5(23.4-36.2)$ \\
\hline Hyperactivity/inattention & 180 & $11.0(9.6-12.7)$ & 179 & $11.0(9.6-12.6)$ & 0.949 & $8.5(7.1-10.0)$ & $31.1(24.4-38.4)$ \\
\hline $\begin{array}{l}\text { Peer relationship } \\
\text { problems }\end{array}$ & 202 & $12.4(10.8-14.1)$ & 182 & $11.2(9.7-12.8)$ & 0.220 & $7.7(6.4-9.2)$ & $35.6(29.0-42.7)$ \\
\hline
\end{tabular}

Note: SDQ = Strengths and Difficulties Questionnaire; $\mathrm{Cl}=$ confidence interval

${ }^{*}$ McNemar test for the difference in prevalence rates between age 6 and age 11

**The cumulative incidence of any difficulty between 6 and 11 years of age was calculated as the proportion of children with any difficulty at 11 years amongst all those who had tested positive at 6 years. The same procedure was used in order to calculate cumulative incidence of emotional symptoms, conduct problems, hyperactivity/inattention and peer relationship problems.

${ }^{* * *}$ The persistence of any difficulty between 6 and 11 years of age was calculated as the proportion of children with any difficulty at 11 years amongst all those who had tested positive at age 6 . The same procedure was used in order to calculate persistence of emotional symptoms, conduct problems, hyperactivity/inattention and peer relationship problems. 
Table S2 Disorders at age 6 as predictors of disorders at age 11, by sex ${ }^{a}$

\begin{tabular}{|c|c|c|c|c|c|c|c|c|}
\hline \multirow[t]{3}{*}{ Assessment at age 6} & \multicolumn{8}{|c|}{ Assessment at age 11} \\
\hline & \multicolumn{2}{|c|}{ Emotional symptoms } & \multicolumn{2}{|c|}{ Conduct problems } & \multicolumn{2}{|c|}{ Hyperactivity/inattention } & \multicolumn{2}{|c|}{$\begin{array}{l}\text { Peer relationship } \\
\text { problems }\end{array}$} \\
\hline & OR $(95 \% \mathrm{Cl})$ & $p$-value & OR $(95 \% \mathrm{Cl})$ & $p$-value & OR $(95 \% \mathrm{Cl})$ & $p$-value & OR $(95 \% \mathrm{Cl})$ & $p$-value \\
\hline \multicolumn{9}{|l|}{ Boys } \\
\hline Emotional symptoms & $4.1(3.0-5.5)$ & $<0.001$ & $2.3(1.6-3.2)$ & $<0.001$ & $2.1(1.6-2.9)$ & $<0.001$ & $2.9(2.1-4.0)$ & $<0.001$ \\
\hline + comorbidity & & & $1.2(0.8-1.8)$ & 0.330 & $1.1(0.8-1.6)$ & 0.650 & $1.7(1.2-2.5)$ & 0.003 \\
\hline Conduct problems & $1.3(0.9-1.7)$ & 0.154 & $5.3(3.9-7.1)$ & $<0.001$ & $3.2(2.4-4.2)$ & $<0.001$ & $2.3(1.7-3.1)$ & $<0.001$ \\
\hline + comorbidity ${ }^{b}$ & $0.7(0.5-1.0)$ & 0.050 & & & $1.4(1.0-1.9)$ & 0.056 & $1.2(0.9-1.8)$ & 0.246 \\
\hline Hyperactivity/inattention & $1.8(1.3-2.4)$ & $<0.001$ & $3.6(2.7-4.9)$ & $<0.001$ & $7.4(5.6-9.8)$ & $<0.001$ & $2.3(1.7-3.2)$ & $<0.001$ \\
\hline + comorbidity & $1.3(0.9-1.9)$ & 0.128 & $1.8(1.3-2.6)$ & $<0.001$ & & & $1.3(0.9-1.9)$ & 0.133 \\
\hline Peer relationship problems & $2.6(1.9-3.4)$ & $<0.001$ & $2.6(1.9-3.5)$ & $<0.001$ & $2.4(1.8-3.2)$ & $<0.001$ & $5.5(4.1-7.4)$ & $<0.001$ \\
\hline + comorbidity ${ }^{b}$ & $2.0(1.4-2.7)$ & $<0.001$ & $1.5(1.1-2.2)$ & 0.023 & $1.5(1.1-2.0)$ & 0.025 & & \\
\hline \multicolumn{9}{|l|}{ Girls } \\
\hline Emotional symptoms & $3.9(2.9-5.3)$ & $<0.001$ & $2.4(1.7-3.5)$ & $<0.001$ & $2.3(1.6-3.3)$ & $<0.001$ & $3.8(2.7-5.3)$ & $<0.001$ \\
\hline+ comorbidity $^{b}$ & & & $1.6(1.1-2.3)$ & 0.028 & $1.5(1.0-2.3)$ & 0.055 & $2.2(1.5-3.3)$ & $<0.001$ \\
\hline Conduct problems & $1.9(1.4-2.6)$ & $<0.001$ & $4.2(3.0-5.9)$ & $<0.001$ & $3.5(2.4-5.0)$ & $<0.001$ & $3.3(2.3-4.7)$ & $<0.001$ \\
\hline+ comorbidity $^{b}$ & $1.2(0.8-1.7)$ & 0.317 & & & $2.0(1.3-3.0)$ & 0.001 & $1.6(1.0-2.5)$ & 0.031 \\
\hline Hyperactivity/inattention & $1.8(1.3-2.5)$ & 0.001 & $3.2(2.2-4.7)$ & $<0.001$ & $4.9(3.4-7.0)$ & $<0.001$ & $2.2(1.5-3.3)$ & $<0.001$ \\
\hline+ comorbidity $^{b}$ & $1.3(0.9-1.9)$ & 0.138 & $2.0(1.3-3.0)$ & $<0.001$ & & & $1.3(0.8-2.1)$ & 0.240 \\
\hline Peer relationship problems & $2.0(1.5-2.8)$ & $<0.001$ & $2.3(1.6-3.4)$ & $<0.001$ & $2.0(1.4-3.0)$ & $<0.001$ & $6.6(4.7 ; 9.4)$ & $<0.001$ \\
\hline + comorbidity & $1.4(1.0-1.9)$ & 0.088 & $1.4(0.9-2.2)$ & 0.106 & $1.3(0.8-2.0)$ & 0.323 & & \\
\hline
\end{tabular}

${ }^{a} n=3362$ individuals with data available for evaluations at age 6 and age 11

${ }^{b}$ Analyses conducted to control for concurrent age 6 comorbidity in the het 
"Lá fora, amor Uma rosa morreu Uma festa acabou Nosso barco partiu Eu bem que mostrei a ela O tempo passou na janela Só Carolina não viu" Chico Buarque 


\section{CONSIDERAÇÕES FINAIS}

O presente estudo observou que a prevalência de qualquer transtorno psiquiátrico no início da adolescência, de acordo com o DSM-5, foi de 13,2\%. Foram preditores dos transtornos psiquiátricos as variáveis: sexo masculino, baixa escolaridade materna, tabagismo durante a gestação, sintomas de transtorno do humor durante a gestação, sintomas depressivos crônicos e graves maternos nos primeiros anos de vida do adolescente, escore de Apgar 5 min $<7$ no nascimento e parto prematuro.

Para este estudo, foram formuladas três hipóteses norteadoras. A primeira delas considerou que a taxa de prevalência de transtornos psiquiátricos aos 11 anos seria de aproximadamente 14\%. Tal hipótese vinculou-se ao objetivo geral e ao primeiro objetivo específico relativo à prevalência dos transtornos psiquiátricos em adolescentes. Diante dos resultados obtidos, considerou-se que a hipótese foi confirmada, uma vez que se verificou que a prevalência de transtornos psiquiátricos do atual estudo encontra-se próxima a prevalência de transtornos psiquiátricos em adolescentes apontada em estudos nacionais e internacionais de base populacional, inclusive em uma recente revisão sistemática e meta-análise que incluiu 27 países e diversos métodos de avaliação dos transtornos psiquiátricos (Polanczyk et al. 2015).

A segunda hipótese propunha que a continuidade homotípica dos transtornos psiquiátricos aos 11 anos seria mais frequente. Esta hipótese articulou-se com o objetivo geral e foi respondida no segundo produto desta tese. Com base nos dados verificados, considerou-se que tal hipótese foi confirmada, pois houve continuidade homotípica significativa entre os seis e 11 
anos de idade nos transtornos de ansiedade, hiperatividade e transtornos de conduta. Para o presente estudo, os transtornos psiquiátricos foram agrupados em quatro grandes grupos de diagnóstico: transtornos de ansiedade, transtornos depressivos, TDAH e transtorno conduta e oposição. Verificou-se que, durante a transição da infância para a adolescência precoce, houve continuidade homotípica para os sintomas emocionais, problemas de conduta, hiperatividade/ desatenção e problemas de relacionamento entre pares.

A terceira hipótese referiu que as variáveis biológicas, clínicas e sociodemográficas consideradas mais negativas, segundo a literatura, estariam associadas à presença de mais indicadores de transtornos psiquiátricos no início da adolescência. Esta hipótese vinculou-se ao segundo objetivo específico do presente estudo de avaliação dos fatores de risco precoces dos transtornos psiquiátricos na adolescência e sua relação com o desenvolvimento dos transtornos psiquiátricos em adolescentes. Frente aos resultados obtidos, considerou-se a confirmação da hipótese, uma vez que as variáveis biológicas, clínicas e, principalmente, sociodemográficas, identificadas como condições de risco, associaram-se à presença de frequência de problemas, especialmente comportamentais. Os adolescentes que pertenciam às famílias com maior índice de bens ( $5^{\circ}$ quintil) tiveram uma frequência quase duas vezes menor de algum transtorno psiquiátrico quando comparados aos adolescentes pertencentes às famílias com nível socioeconômico mais baixo ( $1^{\circ}$ quintil).

Algumas limitações do estudo precisam ser analisadas. O diagnóstico de transtorno psiquiátrico baseou-se apenas nas respostas informadas pela mãe ou responsável. Não foram aplicadas as versões do SDQ junto aos professores ou aos próprios adolescentes. Os relatos de professores e o autorrelato dos 
adolescentes poderiam revelar outros sintomas não reconhecidos pelas mães, contribuindo para uma triagem mais precisa dos transtornos psiquiátricos.

Entre as vantagens do estudo cabe destacar que foi uma pesquisa de base populacional, com entrevistas presenciais em grande amostra de adolescentes. Para avaliação dos transtornos psiquiátricos foi utilizado um instrumento reconhecido internacionalmente, projetado para gerar diagnóstico de transtornos psiquiátricos, validado no Brasil e aplicado por psicólogos treinados que garantiram dados de boa qualidade. Outro ponto de destaque é que no presente estudo foi utilizado o DSM-5, classificação que segue critérios diagnósticos internacionalmente aceitos e que foram recentemente publicados. Ressalta-se ainda a baixa proporção de não respondentes. Além disso, destaca-se o tipo de delineamento adotado, uma vez que os estudos prospectivos de coorte constituem o delineamento mais adequado para se estudar a incidência e continuidade dos transtornos, acompanhando os indivíduos durante anos, décadas ou durante toda a vida.

Os achados do presente estudo mostram a relevância dos transtornos psiquiátricos no início da adolescência. Apresentar a prevalência dos transtornos mentais, bem como fatores de vulnerabilidade e de proteção, auxilia no delineamento de políticas de saúde, na distribuição de recursos, assim como na prevenção e no tratamento de casos diagnosticados (Anselmi et al. 2008). As crianças e os adolescentes com algum transtorno psiquiátrico são particularmente vulneráveis à exclusão social, discriminação, dificuldades no aprendizado, comportamentos de risco e problemas de saúde física. Além disso, os transtornos mentais identificados na infância e adolescência tendem a persistir, aumentando o risco para outros problemas ao longo da vida. Desta 
forma, os resultados apresentados nesta tese fornecem evidências para o desenvolvimento de estratégias de prevenção, promoção e recuperação da saúde mental durante a infância e a adolescência, reduzindo a lacuna de conhecimento relacionada à falta de estudos de base populacional sobre transtornos psiquiátricos em adolescentes de países de baixa e média renda, além de contribuir para o planejamento de serviços específicos direcionados ao tratamento dos transtornos psiquiátricos nessa faixa etária, minimizando o impacto a curto, médio e longo prazo de tais transtornos durante o curso de vida. 
"Inteligência é a capacidade de se adaptar às mudanças".

Stephen Hawking 


\section{REFERÊNCIAS}

Achenbach TM. Manual for the child behavior checklist/4-18 and 1991 profile. Burlington (VT): University of Vermont; 1991.

Almeida-Filho N. Development and assessment of the QMPI: Brazilian children's behavior questionnaire for completion by parents. Soc Psychiatry. 1981;16(4):205-10. 10.1007/BF00582661

American Psychiatric Association. Diagnostic and Statistical Manual of Mental Disorders, Third Edition (DSM-III). Arlington (VA): APA; 1980.

American Psychiatric Association. Diagnostic and Statistical Manual of Mental Disorders, Fifth Edition (DSM-V). Arlington (VA): APA; 2013.

American Psychiatry Association. Diagnostic and Statistical Manual of Mental disorders - DSM-5. 5th ed. Washington (DC): APA; 2014.

Andrade LHSG, Viana MC, Silveira CM. Epidemiologia dos transtornos psiquiátricos na mulher. Rev Psiq Clín. 2006;33(2):43-54. doi: 10.1590/S0101-60832006000200003

Andreoli SB, Almeida-Filho N, Coutinho ESF, Mari JJ. Identificação de casos psiquiátricos em estudos epidemiológicos: métodos, problemas e aplicabilidade. Rev Saude Publica. 2000;34(5):475-83.

Anselmi L, Barros FC, Teodoro ML, Piccinini CA, Menezes AM, Araujo CL, Rohde LA. Continuity of behavioural and emotional problems from preschool years to pre-adolescence in a developing country. J Child Psychol Psychiatry. 2008 May;49(5):499-507. doi: 10.1111/j.14697610.2007.01865.x

Anselmi L, Piccinini CA, Barros FC, Lopes RS. Psychosocial determinants of behaviour problems in Brazilian preschool children. J Child Psychol Psychiatry. 2004 May;45(4):779-88.

Anselmi L, Fleitlich-Bilyk B, Menezes AM, Araújo CL, Rohde LA. Prevalence 
of psychiatric disorders in a Brazilian birth cohort of 11- year-olds. Soc Psychiatry Psychiatr Epidemiol. 2010 Jan;45(1):135-42. doi: 10.1007/s00127-009-0052-2

Araujo AC, Lotufo Neto F. A nova classificação Americana para os Transtornos Mentais - o DSM-5. Rev Bras Ter Comport Cogn. 2014 abr;16(1):67-82.

Beddington J, Cooper CL, Field J, Goswami U, Huppert FA, Jenkins R, Jones HS, Kirkwood TB, Sahakian BJ, Thomas SM.. The mental wealth of nations. Nature. 2008 Oct 23;455(7216):1057-60. doi: 10.1038/4551057a

Belfer ML. Child and adolescent mental disorders: the magnitude of the problem across the globe. J Child Psychol Psychiatry. 2008 Mar;49(3):22636. doi: 10.1111/j.1469-7610.2007.01855.

Ben-Shlomo Y, Kuh D. A life course approach to chronic disease epidemiology: conceptual models, empirical challenges and interdisciplinary perspectives. Int J Epidemiol. 2002 Apr;31(2):285-93.

Benvegnú LA1, Fassa AG, Facchini LA, Wegman DH, Dall'Agnol MM.. Work and behavioural problems in children and adolescents. Int J Epidemiol. 2005 Dec;34(6):1417-24.

Bordin IAS, Paula C. Estudos populacionais sobre saúde mental de crianças e adolescentes brasileiros. In: Mello MF, Mello AAF, Kohn R, organizadores. Epidemiologia da saúde mental no Brasil. Porto Alegre: Artmed; 2007.

Brasil HHA. Desenvolvimento da versão brasileira da K-SADS-PL (Schedule for Affective Disorders and Schizophrenia for School Aged Children Present and Lifetime Version) e estudo de suas propriedades psicométricas [tese não publicada]. São Paulo: Universidade Federal de São Paulo; 2003.

Breslau N, Chilcoat HD. Psychiatric sequelae of low birth weight at 11 years of age. Biol Psychiatry. 2000 Jun 1;47(11):1005-11.

Brion MJ, Victora C, Matijasevich A, Horta B, Anselmi L, Steer C, Menezes 
AM, Lawlor DA, Davey Smith G. Maternal smoking and child psychological problems: disentangling causal and noncausal effects. Pediatrics. 2010 Jul;126(1):e57-65. doi: 10.1542/peds.2009-2754

Bufferd SJ, Dougherty LR, Carlson GA, Rose S, Klein DN.. Psychiatric disorders in preschoolers: continuity from ages 3 to 6. Am J Psychiatry. 2012 Nov; 169(11):1157-64. doi: 10.1176/1ppi.apj.2012.12020268

Buka SL, Monuteaux M, Earls F. The epidemiology of child and adolescent mental disorders. In: Tsuang MT, Tohen M, eds. Textbook in Psychiatric Epidemiology. 2nd ed. New York, NY: John Wiley and Sons Inc; 2002:629655.

Canino G, Alegría M. Psychiatric diagnosis - is it universal or relative to culture? J Child Psychol Psychiatry. 2008 Mar;49(3):237-50. doi: 10.1111/j.1469-7610.2007.01854.x

Carvalho, A. E. V., Linhares, M. B. M., \& Martinez, F. E. (2001). História de desenvolvimento e comportamento de crianças nascidas pré-termo e baixo peso (<1500g). Psicologia: Reflexão e Crítica, 14(1), 1-33.

Caspi A, Moffitt TE. Gene-environment interactions in psychiatry: joining forces with neuroscience. Nat Rev Neurosci. 2006 Jul;7(7):583-90. doi: $10.1038 / \mathrm{nrn} 1925$

Chassin L, Fora DB, King KM.. Trajectories of alcohol and drug use and dependence from adolescence to adulthood: the effects of familial alcoholism and personality. J Abnorm Psychol. 2004 Nov;113(4):48398.10.1037/0021-843X.113.4.483

Costello EJ, Angold A, Burns BJ, Stangl DK, Tweed DL, Erkanli A, Worthman CM.. The Great Smoky Mountains Study of Youth. Goals, design, methods, and the prevalence of DSM-III-R disorders. Arch Gen Psychiatry. 1996 Dec;53(12):1129-36. PMID: 8956679.

Costello EJ, Copeland W, Angold A. Trends in psychopathology across the adolescent years: what changes when children become adolescents, and 
when adolescents become adults? J Child Psychol Psychiatry. 2011 Oct;52(10):1015-25. doi: 10.1111/j.1469-7610.2011.02446.x

Costello EJ, Mustillo S, Erkanli A, Keeler G, Angold A. Prevalence and development of psychiatric disorders in childhood and adolescence. Arch Gen Psychiatry. 2003 Aug;60(8):837-44.

Cury CR, Golfeto JH. Strengths and difficulties questionnaire (SDQ): a study of school children in Ribeirão Preto. Braz J Psychiatry. 2003 Sep;25(3):13945. doi: 10.1590/S1516-44462003000300005

De Girolamo G, Dagani J, Purcell R, Cocchi A, McGorry PD. Age of onset of mental disorders and use of mental health services: needs, opportunities and obstacles. Epidemiol Psychiatr Sci. 2012 Mar;21(1):47-57.. PMID: 22670412.

Dohrenwend BP, Levav I, Shrout PE, Schwartz S, Naveh G, Link BG, Skodol AE, Stueve A. Socioeconomic status and psychiatric disorders: the causation-selection issue. Science. 1992 Feb 21;255(5047):946-52. doi: 10.1126/science. 1546291

Duarte C, Hoven C, Berganza C, Bordin I, Bird H, Miranda CT.. Child mental health in Latin America: present and future epidemiologic research. Int $J$ Psychiatry Med. 33(3):203-22.

Eaton WW, Merikangas KR. Psychiatric epidemiology: progress and prospects in the year 2000. Epidemiol Rev. 2000;22(1):29-34. doi: 10.1093/oxfordjournals.epirev.a018022

Ensminger ME1, Hanson SG, Riley AW, Juon HS.Maternal psychological distress: adult sons' and daughters' mental health and educational attainment. J Am Acad Child Adolesc Psychiatry. 2003 Sep;42(9):1108-15. doi: 10.1097/01.CHI.0000070261.24125.F8

Erskine HE, Moffitt TE, Copeland WE, Costello EJ, Ferrari AJ, Patton G, Degenhardt L, Vos T, Whiteford HA, Scott JG.. A heavy burden on young minds: the global burden of mental and substance use disorders in children 
and youth. Psychol Med. 2015 May;45(7):1551-63. doi: $10.1017 /$ S0033291714002888

Fagerlund, A, Autti-Rämö I, Hoyme HE, Mattson SN, Korkman M. . Risk factors for behavioural problems in foetal alcohol spectrum disorders. Acta Paediatr. 2011;100(11):1481-8. doi: 10.1111/j.1651-2227.2011.02354.x

Fallang B, Oien I, Hellem E, Saugstad OD, Hadders-Algra M. Quality of reaching and postural control in young preterm infants is related to neuromotor outcome at 6 years. Pediatr Res. 2005;58(2):347-53. doi: 10.1203/01.PDR.0000170898.60160.09

Ferdinand RF, Verhulst FC, Wiznitzer M. Continuity and change of selfreported problem behaviors from adolescence into young adulthood. $J A m$ Acad Child Adolesc Psychiatry. 1995 May;34(5):680-90. doi: 10.1097/00004583-199505000-00020

Ferrari AJ, Charlson FJ, Norman RE, Patten SB, Freedman G, Murray CJ, Vos T, Whiteford HA. Burden of depressive disorders by country, sex, age, and year: findings from the global burden of disease study 2010. PLoS Med. 2013;10(11):e1001547. doi: 10.1371/journal.pmed.1001547

Fleitlich, B., Cortázar, P. G., \& Goodman, R. Questionário de capacidades e dificuldades (SDQ). Infanto Rev Neuropsiquiatr Infânc Adolesc. 2000;8(1):44-50.

Fleitlich-Bilyk B, Goodman R. Social factors associated with child mental health problems in Brazil: cross sectional survey. BMJ. 2001 Sep 15;323(7313):599-600. PMID: 11557705.

Fleitlich-Bilyk B, Goodman R. The prevalence of child and adolescent psychiatric disorders in southeast Brazil. J Am Acad Child Adolesc Psychiatry. 2004 Jun;43(6):727-34. doi: 10.1097/01.chi.0000120021.14101.ca

Fleming JA, Hsieh CC. Introduction to epidemiologic research methods. In: Tsuang MT, Tohen M. Textbook in psychiatric epidemiology. New York: 
John Wiley \& Sons, Inc. Cap. 2, p.30-42.

Ford T, Goodman R, Meltzer H. The British Child and Adolescent Mental Health Survey 1999: the prevalence of DSM-IV disorders. J Am Acad Child Adolesc Psychiatry. 2003 Oct;42(10):1203-11. doi: 10.1097/00004583200310000-00011

Ford T, Goodman R, Meltzer H. The relative importance of child, family, school and neighbourhood correlates of childhood psychiatric disorder. Soc Psychiatry Psychiatr Epidemiol. 2004 Jun;39(6):487-96. doi: 10.1007/s00127-004-0782-0

Gale CR, Martyn, CN. Birth weight and later risk of depression in a national birth cohort. Br J Psychiatry. 2004 Jan;184:28-33. doi: 10.1192/bjp.184.1.28

Gao Q, Qian Y, He XX, Sun L, Chang WL, Li YL, Cao QJ, Wang YF, Qian QJ.. Childhood predictors of persistent ADHD in early adulthood: Results from the first follow-up study in China. Psychiatry Res. 2015 Dec 30;230(3):905-12. doi: 10.1016/j.psychres.2015.11.025

Goodman R, Ford T, Richards H, Gatward R, Meltzer H. The development and well-being assessment: description and initial validation of an integrated assessment of child and adolescent psychopathology. J Child Psychol Psychiatry. 2000 Jul;41(5):645-55. doi: 10.1111/j.1469-7610.2000.tb02345.x

Goodman R, Scott S.Child psychiatry. Oxford: Blackwell Publishing; 2005.

Goodman R, Slobodskaya H, Knyazev G. Russian child mental health a cross-sectional study of prevalence and risk factors. Eur Child Adolesc Psychiatry . 2005 Feb;14(1):28-33. doi: 10.1007/s00787-005-0420-8

Goodman, R. The Strengths and Difficulties Questionnaire: a research note. J Child Psychol Psychiatry. 1997 Jul;38(5):581-6. doi: 10.1111/j.14697610.1997.tb01545.x 
Goodwin RD, Fergusson DM, Horwood LJ.. Early anxious/withdrawn behaviours predict later internalising disorders. J Child Psychol Psychiatry. 2004 May;45(4):874-83. doi: 10.1111/j.1469-7610.2004.00279.x

Gore FM, Bloem PJ, Patton GC, Ferguson J, Joseph V, Coffey C, Sawyer SM, Mathers CD. Global burden of disease in young people aged 10-24 years: a systematic analysis. Lancet. 2011 Jun 18;377(9783):2093-102. doi: 10.1016/S0140-6736(11)60512-6

Gutt EK. Perfil comportamental e competência social de crianças e adolescentes filhos de mulheres com esquizofrenia [dissertação]. São Paulo: Faculdade de Medicina, Universidade de São Paulo; 2005.

Hackett, R, Hackett L. Child psychiatry across cultures. Int Rev Psychiatry. 1990;11(2-3):225-35. doi: 10.1080/09540269974410

Halpern R, Figueiras ACM. Influências ambientais na saúde mental da criança. J Pediatr. 2004;80(2):104-10. doi: 10.1590/S002175572004000300013

Hayes B, Sharif F. Behavioural and emotional outcome of very low birth weight infants: literature review. J Matern Fetal Neonatal Med. 22(10):84956. doi: $10.1080 / 14767050902994507$

Hill AB. The environment and disease: association or causation? Proc $R$ Soc Med. 1965 May;58(5):295-300. PMC1898525.

Hofstra MB, Van der Ende J, Verhulst FC. Continuity and change of psychopathology from childhood into adulthood: a 14-year follow-up study. $J$ Am Acad Child Adolesc Psychiatry. 2000 Jul;39(7):850-8. doi: 10.1097/00004583-200007000-00013

Huizink AC,; Mulder EJ. Maternal smoking, drinking or cannabis use during pregnancy and neurobehavioral and cognitive functioning in human offspring. Neurosci Biobehav Rev. 2006;30(1):24-41. doi: 10.1016/j.neubiorev.2005.04.005 
Husby SM, Wichstrom L. Interrelationships and Continuities in Symptoms of Oppositional Defiant and Conduct Disorders from Age 4 to 10 in the Community. J Abnorm Child Psychol. 2017 Jul;45(5):947-958. doi: 10.1007/s10802-016-0210-4

Instituto Brasileiro de Geografia e Estatística. Brasil em síntese [Internet]. 2012b [citado 3 nov 2018]. Disponível em: https://www.ibge.gov.br/estatisticas/sociais/populacao.html

Instituto Brasileiro de Geografia e Estatística. Pesquisa Nacional por Amostra de Domicílios: síntese de indicadores 2011. Rio de Janeiro: IBGE; 2012a.

Jacob S, Byrne M, Keenan K. Neonatal Physiological Regulation is Associated With Perinatal Factors: A Study of Neonates Born to Healthy African American Women Living In Poverty. Infant Ment Health J. 2009 Jan 1;30(1):82-94. doi: 10.1002/imhj.20204

Jacobson SW, Jacobson JL, Stanton ME, Meintjes EM, Molteno CD. Biobehavioral markers of adverse effect in fetal alcohol spectrum disorders. Neuropsychology Rev. 2011 Jun;21(2):148-66. doi: 10.1007/s11065-0119169-7

Jarvis E. Insanity and idiocy in Massachusetts: Report of the Commission on Lunacy, 1855. Cambridge: Harvard University Press; 1971.

Kelly YJ, Nazroo JY, McMunn A, Boreham R, Marmot M. Bitrhweigth and behavioural problemas in children: a modifiable effect? Int J Epidemiol. 2001 Feb;30(1):88-94. doi: 10.1093/ije/30.1.88

Kessler R. Epidemiología psiquiátrica: algunos avances recientes y futuras orientaciones. Bull World Health Org (recomplilación de artículos). 2000:78(3):106-15.

Kessler RC, Amminger GP, Aguilar-Gaxiola S, Alonso J, Lee S, Ustun TB. Age of onset of mental disorders: a review of recent literature. Curr Opin Psychiatry. 2007 Jul;20(4):359-64. doi: 10.1097/YCO.0b013e32816ebc8c 
Khashan AS, Abel KM, McNamee R, Pedersen MG, Webb RT, Baker PN, Kenny LC, Mortensen PB. Higher risk of offspring schizophrenia following antenatal maternal exposure to severe adverse life events. Arch Gen Psychiatry. 2008 Feb;65(2):146-52. doi: 10.1001/archgenpsychiatry.2007.20

Kieling C, Baker-Henningham H, Belfer M, Conti G, Ertem I, Omigbodun O, Rohde LA, Srinath S, Ulkuer N, Rahman A.. Child and adolescent mental health worldwide: evidence for action. Lancet. 2011 Oct 22;378(9801):151525. doi: 10.1016/S0140-6736(11)60827-1

Kim-Cohen J, Caspi A, Moffitt TE, Harrington H, Milne BJ, Poulton R. Prior juvenile diagnoses in adults with mental disorder: developmental follow-back of a prospective-longitudinal cohort. Arch Gen Psychiatry. 2003 Jul;60(7):709-17. doi: 10.1001/archpsyc.60.7.709

Lancefield KS, Raudino A, Downs JM, Laurens KR.. Trajectories of childhood internalizing and externalizing psychopathology and psychotic-like experiences in adolescence: A prospective population-based cohort study. Dev Psychopathol. $2016 \quad$ May;28(2):527-36. doi: 10.1017/S0954579415001108

Last JM, Spasoff RA, Harris SS, editors. A dictionary of epidemiology. 4th ed. New York: Oxford University Press; 2000.

Lieberman E1, Gremy I, Lang JM, Cohen AP.Low birthweight at term and the timing of fetal exposure to maternal smoking. Am J Public Health. 1994 Jul;84(7):1127-31. PMCID: PMC1614741.

Linhares MBM, Chimello JT, Bordin MBM, Carvalho AEV, Martinez FE. Desenvolvimento psicológico na fase escolar de crianças nascidas prétermo em comparação com crianças nascidas a termo. Psicol Reflex Crit. 2005;18(1):109-17. doi: 10.1590/S0102-79722005000100015

Lopes CS, Abreu GA, Santos DF, Menezes PR, Carvalho KM, Cunha CF, Vasconcellos MT, Bloch KV, Szklo M.. ERICA: prevalence of common mental disorders in Brazilian adolescents. Rev Saude Publica. 2016 Feb;50 Suppl 1:14s. doi: 10.1590/S01518-8787.2016050006690 
Maragno L, Goldbaum M, Gianini RJ, Novaes HMD, César CLG. Prevalência de transtornos mentais comuns em populações atendidas pelo Programa Saúde da Família (QUALIS) no Município de São Paulo, Brasil. Cad Saude Publica. 2006;22(8):1639-48. doi: $10.1590 /$ S0102311X2006000800012

Martínez-Cruz CF, Poblano A, Fernández-Carrocera LA, Jiménez-Quiróz R, Tuyú-Torres N. Association between intelligence quotient scores and extremely low birth weight in school-age children. Arch Med Res. 2006 Jul;37(5):639-45.doi: 10.1016/j.arcmed.2005.12.001

Mash EJ, Hunsley J. Assessment of child and family disturbance: a developmental-systems approach. In: Mash EJ, Barkley RA, editors. Assessment of childhood disorders. New York: Guilford; 2007. p.3-52.

Mathers CD, Loncar D. Projections of global mortality and burden of disease from 2002 to 2030. PLoS Med. 2006 Nov;3(11):e442. doi: 10.1371/journal.pmed.0030442

Mathiesen KS, Sanson A. Dimensions of early childhood behavior problems: stability and predictors of change from 18 to 30 months. J Abnorm Child Psychol. 2000 Feb;28(1):15-31. J Abnorm Child Psychol. 2000 Feb;28(1):15-31. PMID: 10772347.

McGee CL, Riley EP. Social and behavioral functioning in individuals with prenatal alcohol exposure. Int J Disabil Hum Dev. 2007;6(4):369-82. doi: 10.1515/IJDHD.2007.6.4.369

Medeiros EM, Ferreira Filha, Vianna RPT. Epidemiology of mental health and insanity: a review of literature. Online Braz J Nurs. 2016 abr;5(1). doi: 10.5935/1676-4285.2006138

Medronho RA, Carvalho DM, Bloch RR, Werneck GL. Epidemiologia. São Paulo: Atheneu; 2003. Cap.2, p. 17-60.

Merikangas KR, He JP, Burstein M, Swanson SA, Avenevoli S, Cui L, Benjet C, Georgiades K, Swendsen J. Lifetime prevalence of mental disorders in 
U.S. adolescents: results from the National Comorbidity Survey Replication-Adolescent Supplement (NCS-A). J Am Acad Child Adolesc Psychiatry. 2010 Oct;49(10):980-9. doi: 10.1016/j.jaac.2010.05.017

Merikangas, KR, Nakamura EF, Kessler RC. Epidemiology of mental disorders in children and adolescents. Dialogues Clin Neurosci. 2009;11(1):7-20. PMCID: PMC2807642.

Miranda CA, Torasconi CV, Scortegogna SA. Estudos Epidemiológicos dos Transtornos Mentais. Aval Psicol. 2008 ago;7(2):249-57.

Moffitt TE, Arseneault L, Jaffee SR, Kim-Cohen J, Koenem KC, Odgers CL, Slutske WS, Viding E. Research review: DSM-IV conduct disorder: research needs for a evidence base. J Child Psychol Psychiatry. 2008 Jan;49(1):3-33. doi: 10.1111/j.1469-7610.2007.01823.x

Moffitt TE, Caspi A. Childhood predictors differentiate life-course persistent and adolescence-limited antisocial pathways among males and females. Dev Psychopathol. 2001 Spring;13(2):355-75. PMID: 11393651.

Moffitt TE; E-Risk Study Team. Teen-aged mothers in contemporary Britain. J Child Psychol Psychiatry. 2002 Sep;43(6):727-42. doi: 10.1111/14697610.00082

Mullick MS, Goodman R. The prevalence of psychiatric disorders among 510 year olds in rural, urban and slum areas in Bangladesh: an exploratory study. Soc Psychiatry Psychiatr Epidemiol. 2005 Aug;40(8):663-71. doi: 10.1007/s00127-005-0939-5

Noronha MGRCS, Cardoso PS, Moraes TNP, Centa ML. Resiliência: nova perspectiva na promoção da saúde da família? Cienc Saude Col. 2009;14(2):497-506. doi: 10.1590/S1413-8123200900020001

Pacheco J, Alvarenga P, Reppold C, Piccinini CA, Hutz CS. Estabilidade do Comportamento Anti-social na Transição da Infância para a Adolescência: Uma Perspectiva Desenvolvimentista. Psicol Reflex Crit. 2005;18(1):55-61. doi: 10.1590/S0102-79722005000100008 
Paley B. Introduction: Fetal alcohol spectrum disorders--shedding light on an unseen disability. Dev Disabil Res Rev. 2009;15(3):167-9. doi: $0.1002 / d d r r .70$

Patel V. Mental health in low- and middle-income countries. Br Med Bull. 2007;81-82:81-96. doi: 10.1093/bmb/ldm010

Patton GC, Coffey C, Carlin JB, Olsson CA, Morley R. Prematurity at birth and adolescent depressive disorder. Br J Psychiatry. 2004 May;184:446-7. PMID: 15123510.

Pesce, RP, Assis SG, Santos N, Oliveira RVC. Risco e proteção: em busca de um equilíbrio promotor de resiliência. Psicol Teor Pesqui. 2004;20(2):135-43. doi: 10.1590/S0102- 37722004000200006

Petresco S, Anselmi L, Santos IS, Barros AJ, Fleitlich-Bilyk B, Barros FC, Matijasevich A. Prevalence and comorbidity of psychiatric disorders among 6-year-old children: 2004 Pelotas Birth Cohort. Soc Psychiatry Psychiatr Epidemiol. 2014;49(6):975-83. doi: 10.1007/s00127-014-0826-z

Philipp J, Zeiler M, Waldherr K, Nitsch M, Dür W, Karwautz A, Wagner G. The Mental Health in Austrian Teenagers (MHAT)-Study: preliminary results from a pilot study. Neuropsychiatr. 2014;28(4):198-207. doi: 10.1007/s40211-014-0131-9

Piche G, Bergeron L, Cyr M, Berthiaume C.Maternal Lifetime Depressive/Anxiety Disorders and Children's Internalizing Symptoms: The Importance of Family Context. J Can Acad Child Adolesc Psychiatry. 2011 Aug;20(3):176-85. PMCID: PMC3143693.

Polanczyk GV, Salum GA, Sugaya LS, Caye A, Rohde LA. Annual research review: A meta-analysis of the worldwide prevalence of mental disorders in children and adolescents. J Child Psychol Psychiatry.. 2015 Mar;56(3):34565. doi: $10.1111 /$ jcpp. 12381 
Reiss F. Socioeconomic inequalities and mental health problems in children and adolescents: a systematic review. Soc Sci Med. 2013 Aug;90:24-31. doi: 10.1016/j.socscimed.2013.04.026

Riley RD, Higgins JPT, Deeks JJ. Interpretation of random effects metaanalyses. BMJ. 2011 Feb 10;342:d549. doi: 10.1136/bmj.d549

Robbins LN, Regier DA, editors. Psychiatric disorders in America: the epidemiologic catchment area study. New York: Free Press Toronto; 1991.

Rodney HE, Mupier R, Crafter B. Predictors of alcohol drinking among African American adolescents: Implications for violence prevention. J Negro Educ. 1996 Auttumn;65(4):434-44. doi: 10.2307/2967146

Rothman KJ, Greenland S, Lash, T.L. Epidemiologia moderna. 3a ed. Porto Alegre: Artmed; 2011.

Rothman KJ, Greenland S. Modern Epidemiology. 2a ed. Phila- delphia: Lippincott Raven; 1998.

Rutter M, Kim-Cohen J, Maughan B. Continuities and discontinuities in psychopathology between childhood and adult life. J Child Psychol Psychiatry. 2006 Mar-Apr;47(3-4):276-95. doi: 10.1111/j.14697610.2006.01614.x

Rutter M. Poverty and child mental health: natural experiments and social causation. JAMA. 2003 Oct 15;290(15):2063-4. doi: 10.1001/jama.290.15.2063

Rutter M. Psychopathology and development: I. Childhood antecedents of adult psychiatric disorder. Aust N Z J Psychiatry. 1984 Sep;18(3):225-34. doi: 10.3109/00048678409161295

Saigal S, den Ouden L, Wolke D, Hoult L, Paneth N, Streiner DL, Whitaker A, Pinto-Martin J. School-age outcomes in children who were extremely low birth weight from four international population-based cohorts. Pediatrics. 2003 Oct;112(4):943-50. PMID: 14523190. 
Santos IS, Barros AJ, Matijasevich A, Domingues MR, Barros FC, Victora CG. Cohort profile: the 2004 Pelotas (Brazil) birth cohort study. Int $J$ Epidemiol. 2011 Dec;40(6):1461-8. doi: 10.1093/ije/dyq130

Santos IS, Barros AJ, Matijasevich A, Zanini R, Chrestani Cesar MA, Camargo-Figuera FA, Oliveira IO, Barros FC, Victora CG. Cohort profile update: 2004 Pelotas (Brazil) Birth Cohort Study. Body composition, mental health and genetic assessment at the 6 years follow-up. Int $J$ Epidemiol. 2014 Oct;43(5):1437-1437a-f. doi: 10.1093/ije/dyu144

Sapienza G, Pedromônico MRM. Risco, proteção e resiliência no desenvolvimento da criança e do adolescente. Psicol Estud. 2005;10(2):209-16. doi: 10.1590/S1413-73722005000200007

Souza ER, Cerveny CMO. Resiliência: introdução à compreensão do conceito e suas implicações no campo da psicologia. Rev Cienc Hum. 2006;12(2):21-9.

Souza J, Jeronymo DVZ, Carvalho AMP. Maturidade emocional e avaliação comportamental de crianças filhas de alcoolistas. Psicol. Estud. 2005;10(2):191-9. doi: 10.1590/S1413-73722005000200005

van Oort FV, van der Ende J, Wadsworth ME, Verhulst FC, Achenbach TM. Cross-national comparison of the link between socioeconomic status and emotional and behavioral problems in youths. Soc Psychiatry Psychiatr Epidemiol. 2011 Feb;46(2):167-72. doi: 10.1007/s00127-010-0191-5

Vieira MEB, Linhares MBM. Desenvolvimento e qualidade de vida em crianças nascidas pré-termo em idades pré-escolar e escolar. J Pediatr. 2011;87(4):281-91. doi: 10.1590/S0021-75572011000400003

Vitolo YLC, Fleitlich-Bilyk B, Goodman R, Bordin IASB. Parental beliefs and child-rearing attitudes and mental health problems among schoolchildren. Rev Saude Publica. 2005 Oct;39(5):716-24. doi: 10.1590/S003489102005000500004 
Vostanis P. Strenghts and Difficulties Questionnaire: research and clinical aplications. Curr Opin Psychiatry. 2006 Jul;19(4):367-72. doi: 10.1097/01.yco.0000228755.72366.05

Walker SP, Wachs TD, Gardner JM, Lozoff B, Wasserman GA, Pollitt E, Carter JA; International Group Development Steering Group. Child development: risk factors for adverse outcomes in developing countries. Lancet. 2007 Jan 13;369(9556):145-57. doi: 10.1016/S01406736(07)60076-2

Weissman MM, Bland RC, Canino GJ, Faravelli C, Greenwald S, Hwu HG, Joyce PR, Karam EG, Lee CK, Lellouch J, Lépine JP, Newman SC, RubioStipec M, Wells JE, Wickramaratne PJ, Wittchen H, Yeh EK. Cross-national epidemiology of major depression and bipolar disorder. JAMA. 1996;276(4):293-9. doi: 10.1001/jama.1996.03540040037030

Weissman MM, Warner V, Wickramaratne PJ, Kandel DB. Maternal smoking during pregnancy and psychopathology in offspring followed to adulthood. $J$ Am Acad Child Adolesc Psychiatry. 1999 Jul;38(7):892-9. doi: 10.1097/00004583-199907000-00020

Whiteford HA, Degenhardt L, Rehm J, Baxter AJ, Ferrari AJ, Erskine HE, Charlson FJ, Norman RE, Flaxman AD, Johns N, Burstein R, Murray CJ, Vos T. Global burden of disease attributable to mental and substance use disorders: findings from the Global Burden of Disease Study 2010. Lancet. 2013 Nov 9;382(9904):1575-86. doi: 10.1016/S0140-6736(13)61611-6

Whiteside-Mansell L, Bradley RH, Casey PH, Fussell JJ, Conners-Burrow NA. Triple risk: do difficult temperament and family conflict increase the likelihood of behavioral maladjustment in children born low birth weight and preterm? J Pediatr Psychol. 2009 May;34(4):396-405. doi: 10.1093/jpepsy/jsn089

Wiles NJ, Peters TJ, Heron J, Gunnell D, Emond A, Lewis G. Fetal growth and childhood behavioral problems: results from the ALSPAC Cohort. Am J Epidemiol. 2006 May 1;163(9):829-37. doi: 10.1093/aje/kwj108 
World Health Organization. Strengthening mental health promotion. Geneva: WHO; 2000. (Fact sheet no.220).

World Health Organization. The World health report 2001: Mental health: new understanding, new hope. Geneva: WHO; 2001. 